\title{
Assessing Inclusivity of Women at NIST: A Comprehensive Examination of HR Data, In-Depth Interviews and Survey Data
}

\author{
Mary Theofanos \\ Sandra Spickard Prettyman
}

This publication is available free of charge from:

https://doi.org/10.6028/NIST.IR.8376

National Institute of Standards and Technology U.S. Department of Commerce 


\title{
Assessing Inclusivity of Women at NIST: A Comprehensive Examination of HR Data, In-Depth Interviews and Survey Data
}

\author{
Mary F. Theofanos \\ Office of Data and Informatics \\ Material Measurement Laboratory \\ Sandra Spickard Prettyman \\ Culture Catalyst, LLC
}

This publication is available free of charge from:

https://doi.org/10.6028/NIST.IR.8376

July 2021

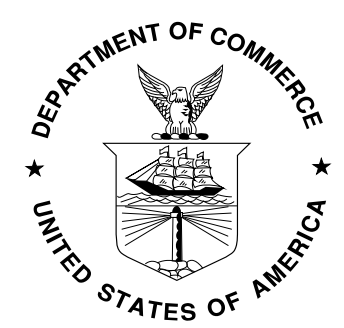

U.S. Department of Commerce

Gina M. Raimondo, Secretary

National Institute of Standards and Technology James K. Olthoff, Performing the Non-Exclusive Functions and Duties of the Under Secretary of Commerce for Standards and Technology \& Director, National Institute of Standards and Technology 
Certain commercial entities, equipment, or materials may be identified in this document in order to describe an experimental procedure or concept adequately. Such identification is not intended to imply recommendation or endorsement by the National Institute of Standards and Technology, nor is it intended to imply that the entities, materials, or equipment are necessarily the best available for the purpose.

National Institute of Standards and Technology Interagency or Internal Report 8376 Natl. Inst. Stand. Technol. Interag. Intern. Rep. 8376, 95 pages (July 2021)

This publication is available free of charge from: https://doi.org/10.6028/NIST.IR.8376 


\begin{abstract}
In the fall of 2019, the National Institute of Standards and Technology (NIST) funded three studies to better understand equity and inclusivity. This report is a comprehensive integration and summary of the sequential, exploratory mixed-methods study designed to provide an indepth look at the population of NIST federal employees to identify factors, attitudes, and processes that might result in gender-specific barriers for women at NIST. This final report integrates the findings from the three phases providing a holistic view by focusing on the themes that emerged from the qualitative in-depth interviews and examining the extent to which and ways in which the quantitative survey and human resources data confirm or challenge the qualitative results. An overwhelming theme and feeling of frustration became evident during the examination of the data from the holistic perspective. NIST staff expressed frustration and exhaustion with the focus on women and not other groups, with the entire subject matter of diversity and inclusivity, with the slow pace of change, and some with reverse discrimination. Overall, the three phases align and provide a consistent view on the differences in how women and men experience NIST, the culture, diversity, and inclusivity.
\end{abstract}

\title{
Key words
}

Diversity, Inclusivity, Gender, In-Depth Interviews, Survey, Mixed-Methods. 


\section{Table of Contents}

1. Introduction............................................................................................................................. 1

2. Methodology ................................................................................................................... 2

2.1. HR Demographic Workforce Data Collection and Analysis...........................................3

2.2. Qualitative Data Collection and Analysis .................................................................................3

2.3. Quantitative Data Collection and Analysis ............................................................................... 4

2.4. Mixed Methods Data Analysis ....................................................................................................... 4

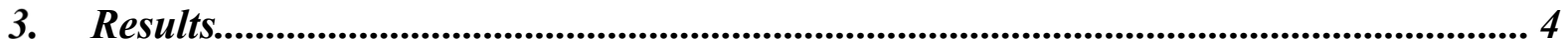

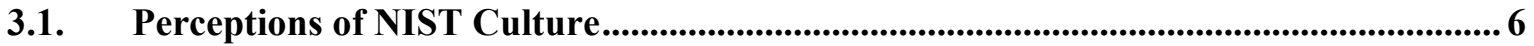

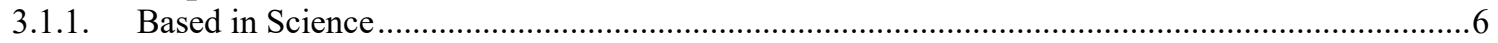

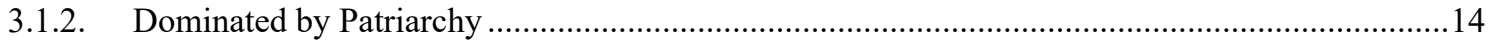

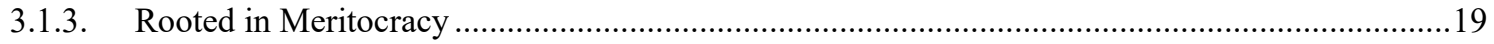

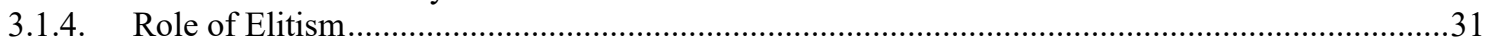

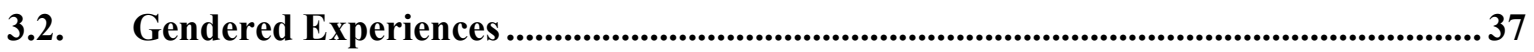

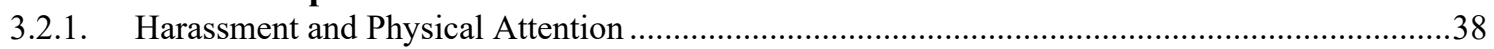

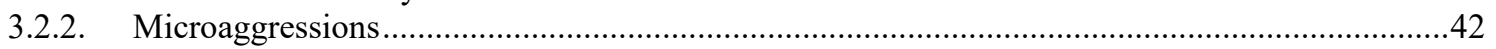

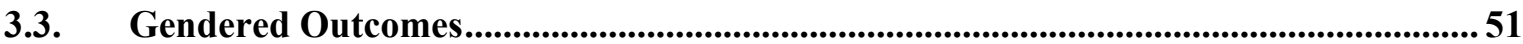

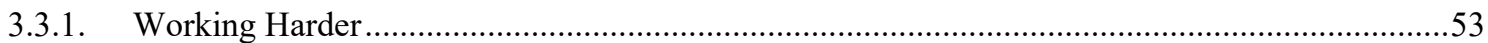

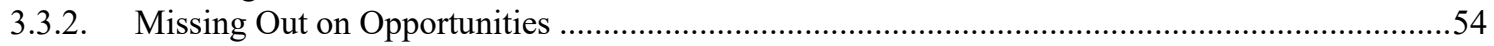

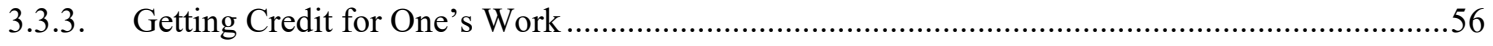

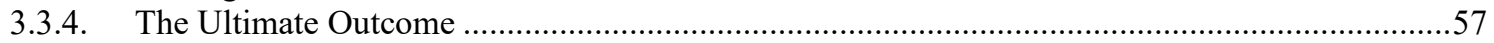

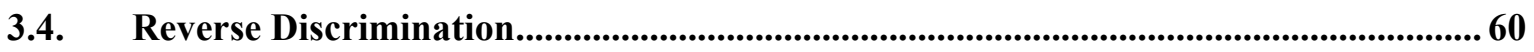

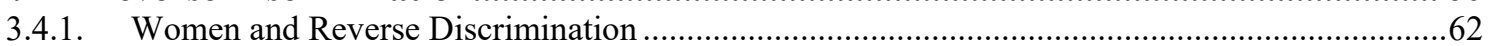

3.5. Ultimate Outcome: The Perpetuation of the Boys' Club and the Chilly Climate. Error! Bookmark not defined.

3.6. Perceptions of NIST's Commitment to Diversity: The Ongoing Need for Change .... 67

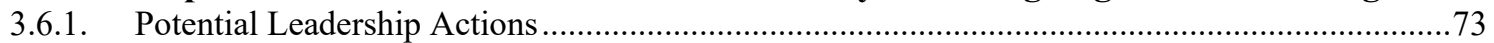

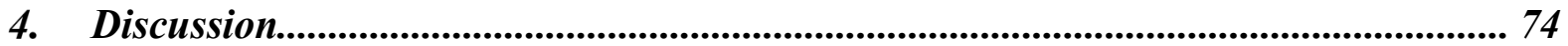

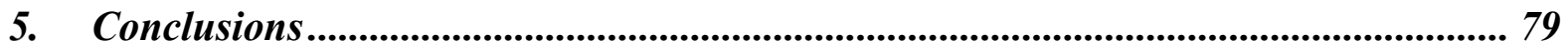

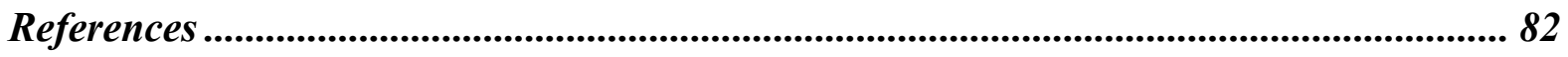

Appendix A: Survey Notation and Numbering........................................................................ 84 


\section{List of Figures}

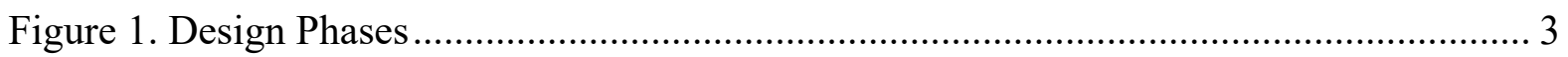

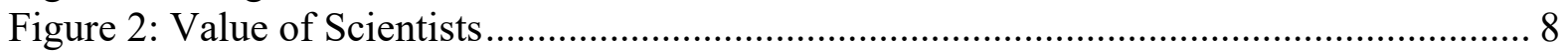

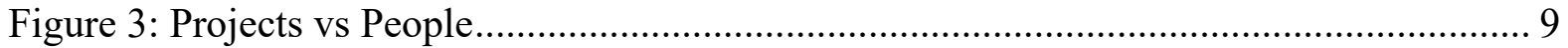

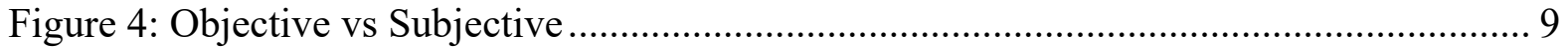

Figure 5: Value of teamwork ………………………................................................ 12

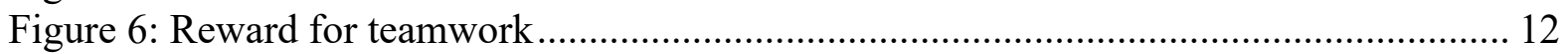

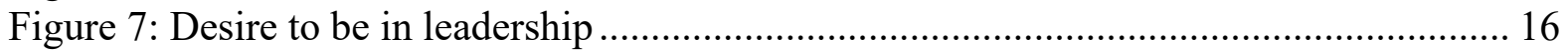

Figure 8: Opportunity to move into leadership............................................................... 16

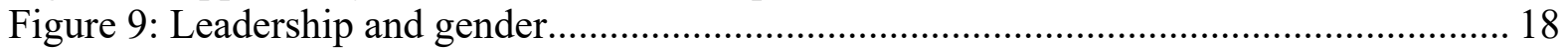

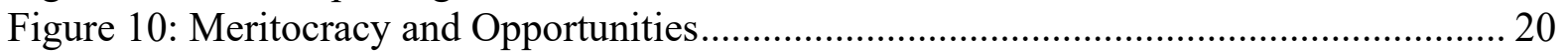

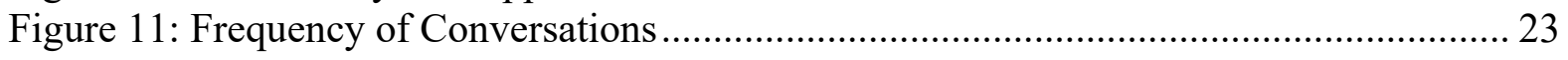

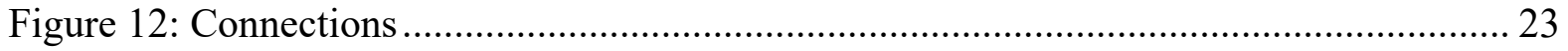

Figure 13: Opportunities for Advancement and Promotion ................................................. 29

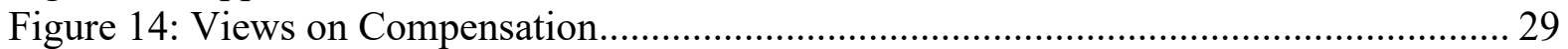

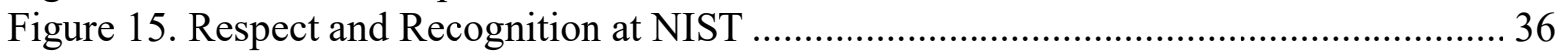

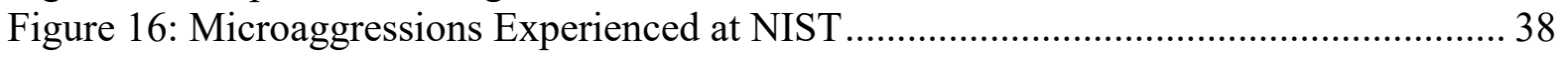

Figure 17: Percentages of those Experiencing Harassment ................................................... 39

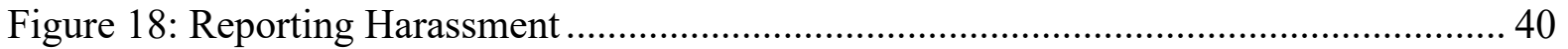

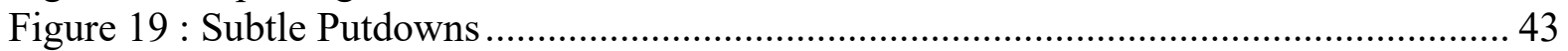

Figure 20: Questioning Competence ………………...................................................... 45

Figure 21: Treated Differently Based on Gender ................................................................ 46

Figure 22: Witnessed Different Treatment Based on Gender................................................ 47

Figure 23: Women with Children and Commitment to Careers ............................................. 49

Figure 24: Comparison of Value, Respect and Treatment.................................................... 51

Figure 25: Women Work Harder for Recognition.............................................................. 53

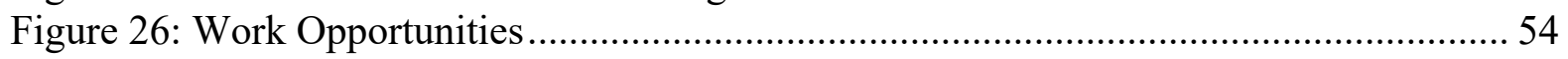

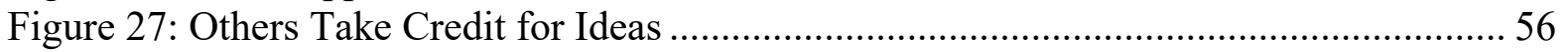

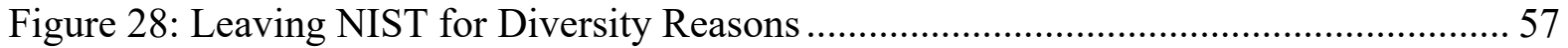

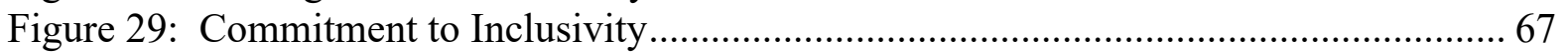

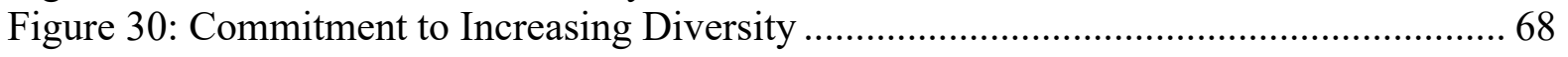

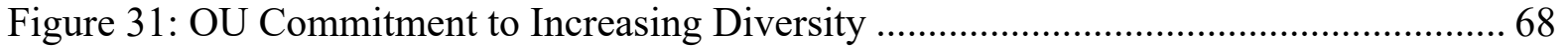

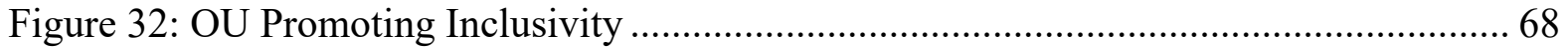




\section{Introduction}

To better understand equity and inclusivity at the National Institute of Standards and Technology (NIST), the Associate Director for Laboratory Programs (ADLP) funded three studies in 2019. While there are many populations at NIST that could benefit from a study examining diversity, inclusivity and equity, this project focused on gender, specifically women and NIST's core value of inclusivity. Given the one-year time frame it was necessary to limit the scope of the effort to one population and to federal employees to ensure a rigorous and completable research project. The approach may serve as a guide and provide a template to study other populations at NIST.

The purpose of this research effort is to explore the experiences of NIST federal employees to identify the ways in which differential opportunities and outcomes might exist related to gender. The goal is to provide the organization with data about how inequities manifest themselves to provide more equitable and inclusive experiences for all employees. A multiphase, mixed methods research project was conducted in order to provide an understanding of the factors that influence experiences of NIST federal employees.

The study was designed in three phases. Phase 1 consisted of an in-depth statistical analysis of approximately 10 years of human resources (HR) data focusing on composition and demographics of the workforce at NIST. This phase examined how women's salaries, promotions, awards, and other indicators compare to those of men in the organization. The results of the analysis of the HR data informed the development of an interview protocol for Phase 2. This qualitative component consisted of in-depth interviews with 40 employees from a range of positions and career paths. The qualitative data from Phase 2 informed the development of an organization-wide quantitative survey used in Phase 3. The use of a larger-scale survey allowed for greater representation from all federal employees across all of NIST to provide a more comprehensive view of experiences, beliefs and perceptions of the NIST federal staff. The greater representation provided the ability to confirm and determine the extent of the findings from Phase 2 of the study, which identified differential experiences for women and men with respect to NIST's culture based in science and meritocracy.

A series of reports from each of these phases highlight the specific analyses of the data for that phase. The Phase 1 report [1] presents the analytical approach and statistical methods used to identify trends of gender differences in the areas of hiring, pay band, annual salary, and supervisory level across NIST. The Phase 2 report [2], describes the qualitative phase of the research design - the in-depth interview research protocol, the participant demographics, the qualitative approach used to analyze the in-depth interview data, and the results. The results focused on three interconnected themes that emerged from the data: 1) a culture at NIST that creates a "Chilly Climate" for women; 2) gendered experiences that contribute to and are outcomes of that Chilly Climate; and 3) gendered outcomes that influence women's experiences in the organization. The Phase 3 report [3] describes the quantitative phase of the research design. The report describes the survey development, participant demographics and the results from the survey. This final report integrates the findings from the three phases to provide a holistic view of the findings focusing on how the data converge. 


\section{Methodology}

Qualitative research methodologies are used to explore why or how a phenomenon occurs, to develop a theory, or describe the nature of an individual's experience. Quantitative methodologies address questions about causality, generalizability, or magnitude of effect [4]. Mixed-method research designs take advantage of the strengths of both qualitative and quantitative methodologies. Data are collected using both methods, analyzed separately, and integrated to address the research questions [5].

This study used an exploratory sequential mixed-method research design to assess NIST federal employees' beliefs, perceptions, and experiences related to gender equity and inclusion and to identify the ways in which NIST culture (including organizational practices and policies as well as broader cultural values and beliefs) contributes to gender inequities and a lack of inclusion, if at all. The research was guided by the following research questions:

\section{Overarching guiding questions}

1. What are the barriers and facilitators, if any, that impact women's opportunities at NIST?

2. In what way does the quantitative and qualitative data converge or diverge?

\section{Qualitative Questions}

1. How do women and men describe their work experiences, their work environments, and their work opportunities at NIST?

a. In what ways are the work experiences, work environments, and work opportunities similar and/or different for women and men?

2. What practices/systems contribute to limiting or facilitating career opportunities for women and men at NIST.

\section{Quantitative Questions}

1. What are the differences, if any, in the ways in which men and women experience work at NIST?

2. What, if any, gender inequities exist at NIST?

a. To what extent do employees at NIST perceive that gender inequities exist?

3. In what ways, if at all, does NIST culture contribute to a lack of inclusivity? This question was added after the analysis of the in-depth interviews based on the qualitative data.

In an exploratory design, qualitative data is collected and analyzed, and themes are used to drive the development of a quantitative instrument to further explore the research problem [5]. The design approach implies that three stages of analyses are performed. The first is conducted after the qualitative data is collected, the second after the quantitative data is collected, and the third at the integration phase (Figure 1). The integration phase connects the two branches of data and extends the qualitative exploratory findings [5]. 
This report documents the final integration phase of the research, presenting the results of the overarching analysis of the effects of gender on opportunities at NIST from the three phases of the research study.

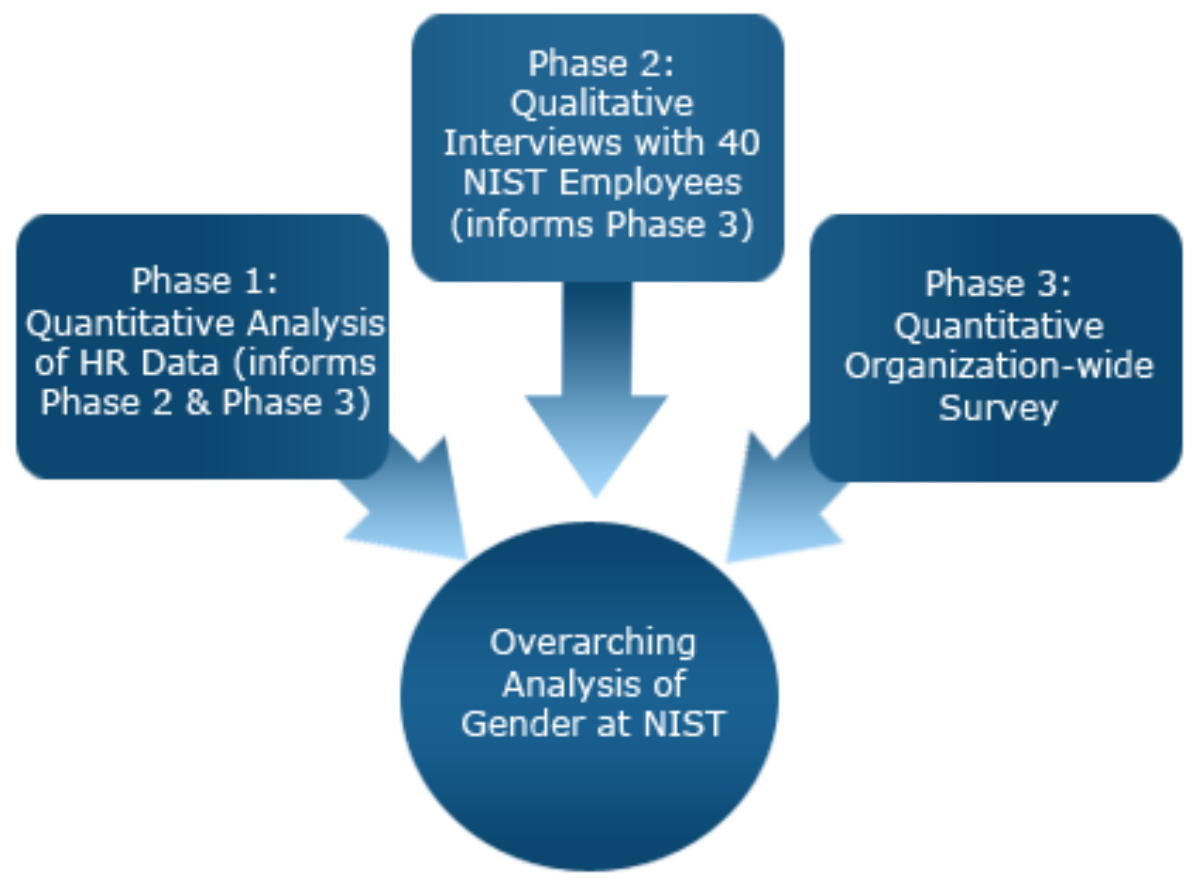

Figure 1. Design Phases

\subsection{HR Demographic Workforce Data Collection and Analysis}

In the first phase of the effort, data was collected from HR on all federal employees from 2010 through 2019. The data included approximately 30,000 records that included annual salary, annual bonus amount, pay scale, pay plan, operating unit (OU), position title, gender, age, NIST assigned workplace location (duty station), employment status, years of service at NIST and in the Federal Government, supervisory status, performance rating and education level. The data was analyzed using descriptive and inferential statistics. A complete description of the data collection and analysis strategies was documented in the HR report $[1]$.

\subsection{Qualitative Data Collection and Analysis}

In the qualitative phase of the study, data was collected from NIST federal employees. Primary qualitative data included semi-structured interviews with a random sample from the 2019 HR data $(N=22)$, and a purposeful sample $(N=18)$. An interview protocol was used to guide the semi-structured interviews. The interviews focused on participants' career journeys, work environments, relationships with colleagues, experiences with bias, and support systems. The qualitative data (transcripts) were coded using grounded theory 
techniques of open, axial, and selective coding relying on both a priori and emergent codes and ideas; memoing and constant comparative methods were also used to elicit themes. A complete description of the qualitative collection and analysis strategies was documented in Qualitative Report [2].

\subsection{Quantitative Data Collection and Analysis}

Data from the qualitative phase were used to develop a survey instrument ${ }^{1}$ for the second quantitative phase of the study. The survey measured the following dimensions: NIST's commitment to diversity and inclusivity; perceptions of NIST's culture; gendered experiences at NIST; interactions at NIST; beliefs about NIST; and demographics. Questions were built from the themes that emerged from the qualitative data analysis. The survey was deployed to all current NIST federal employees in to generalize the findings from the initial qualitative research. A total of 1,093 respondents completed the survey for a $33.2 \%$ response rate. Survey data were analyzed using descriptive and inferential statistics. A complete description of the quantitative data collection and analysis strategies utilized was reported in survey report [3].

\subsection{Mixed-Methods Data Analysis}

According to Creswell and Plano Clark [5] using a combination of qualitative and quantitative data collection methods within a study is not sufficient to categorize a study as 'mixed methods.' The defining characteristic of mixed-methods research is the integration of the strands of data. In this study, integration of the data began at the design level by using a sequential design where the results from the qualitative phase of the research were used to build the next stage of the research design. Integration involved using the qualitative results, specifically the themes, to design the quantitative survey. Integration also occurs as the sets of data are integrated into conclusions. At this stage, the quantitative data refines the interpretation of the qualitative data and the project as a whole.

\section{Results}

Several conventions are used in the presentation of the results that are key to understanding the following sections. All quotes in this report, including quotes from interview participants and open-ended responses from participants of the survey, are verbatim and are presented in blue, indented text. Interview quotes come directly from the participant transcripts of the audio recorded interviews. Survey quotes come directly from the text boxes of the survey and area not corrected for grammar or spelling. The associated notations represent the source from the interview transcripts and survey texts. Thus, interview quotes are distinguishable from survey quotes in their notations.

\footnotetext{
${ }^{1}$ Certain commercial entities, equipment, or materials may be identified in this document in order to describe an experimental procedure or concept adequately. Such identification is not intended to imply recommendation or endorsement by the National Institute of Standards and Technology, nor is it intended to imply that the entities, materials, or equipment are necessarily the best available for the purpose.
} 
The interview notation is composed of four parts: the first represents that the quote derives from the in-depth interviews and begins with INT, the second represents the participant number and whether they were from the random sample (begins with a $\mathrm{P} 0$ ) or from the purposeful sample (begins with a P1), the second refers to the gender of the participant (F, M or $\mathrm{O}$ ), and the third represents the time stamp of the quote in the transcript. For example, (INT:P101:M-5:35) refers to a quote from the in-depth interviews, from purposefully selected participant 01 who is male, and the quote appears at time stamp 5:35 in the transcript. These conventions provide assurances of the data's provenance and that the data can quickly and easily be located within the larger datasets when necessary. Any potentially identifiable information within the quotes has been redacted, with redacted text replaced with general terms in parentheses (e.g., (name redacted)).

With respect to the survey data, all of the major survey sections included questions with open-ended responses. Appendix A provides a table cross referencing the sections, section numbers and questions and question numbers. The open-ended text boxes had no character limits. The open-ended responses were analyzed and are presented here where applicable. At the end of each quoted response is a notation that represents a particular survey response. The notation is composed of four parts: the first represents the section from the survey (S) followed by the number of the section, the second represents the question number within that section $(\mathrm{Q})$ followed by a number. The third element is the record ID number from the survey, and the last identifies the gender (female $(\mathrm{F})$, male $(\mathrm{M})$ or other $(\mathrm{O})$ ) of the participant. For example, (S1Q2:P1234:F) represents the survey responses for section 1, question 2 for record ID \#1234, a female participant. Since the surveys were completed anonymously, it is not possible to associate a participant ID to a specific individual.

Throughout the subsequent Results sections, quotes from the interview data are presented where appropriate, along with relevant responses from the open-ended survey questions. The quotes are provided as exemplars to convey participants' perspectives and should not be viewed solely as the thoughts of a single participant. Finally, using a similar convention to the notation for survey quotes, survey questions are referred to as $\mathrm{S} \# \mathrm{Q} \#$ for section followed by the question number. For example, S1Q1 refers to the question number 1 in section 1.

Since participation in the survey was voluntary, none of the questions on the survey required a response. Thus, participants could choose to skip any of the questions and continue to the next question, or they could choose to quit the survey at any point. While 1093 participants completed the survey in its entirety, the number of participants who responded to a particular question varied. Thus, figures and tables include the total number of responses, $n$, for the corresponding question to provide for proper interpretation. The complete results and $n$ 's for each survey question are presented in the Survey Report [3].

The following sections report on the themes that emerged from the qualitative in-depth interviews examining the extent to which and ways in which the quantitative survey results confirm or challenge the qualitative results. 


\subsection{Perceptions of NIST Culture}

This section further examines the perceptions of NIST culture and the themes of excellence in science, patriarchy, meritocracy, and elitism that emerged from the qualitative in-depth interviews in the context of the survey data. In many instances a single survey question may provide insight into multiple topics from perceptions of NIST culture to gendered experiences and gendered outcomes. When survey questions span topics and subcategories, the reader will be referred back to the first instance of the question's relevance.

\subsubsection{Based in Science}

As a scientific organization, NIST culture is dominated by broader ideas about the nature of science. The culture of science is rooted in beliefs about the value of objectivity, rationality, and competition, beliefs which grow out of and also contribute to ideas of meritocracy and elitism. These ideologies contribute to cultural ways of doing and being that appear neutral on the surface yet often privilege men and "masculine" ways of doing and being, which has the ability to produce different experiences and outcomes for women and men. This broader culture of science, and the way it plays out in scientific organizations like NIST, mitigates against seeing gender at all or at the very least against acknowledging the ways in which it helps to maintain unequal systems of access and outcome. Some researchers argue that the culture of science itself contributes to gender inequality and that masculinity is "invisibly embedded" in scientific inquiry at the same time that science purports to be about "value-free inquiry and purely meritocratic standards of achievement [6,p.8]. As foundational beliefs about the nature of science, they contribute to an acceptance of the status quo and a resistance to change. Science is also seen as the pursuit of excellence, which can contribute to an acceptance of the status quo and difficulty in seeing the need for change. Why would you change something that is already excellent?

The culture of science contributes to and supports both institutional and individual forms of discrimination, in this case based on gender (although it clearly also contributes to and supports other forms of discrimination). This discrimination is often obscured by beliefs about how excellence, objectivity, rationality, and competition are inherent to science.

\subsubsection{Excellence and Intellect: Science as King}

NIST is known as one of the premiere science institutions in the world, employing many of the world's finest scientists. The organization prides itself on a culture of excellence related to its scientific mission, something that was mentioned often in both the interviews and the open-ended survey data.

Our mission is critical to the United States. We are engaged in cutting-edge-- I mean, things I don't even understand myself. So it's got to be cutting-edge. You know what I mean? It's just we're helping society. (INT:P006:F-24:40)

I'm proud of NIST's reputation and it's contribution to science. (S8Q1a:P2366:F)

NIST is a highly respected organization. I am proud of working here. (S8Q1a:1557:O). 
Overwhelmingly, participants speak about NIST as "cutting-edge," "important," and "respected," leading them to be proud of the organization and the work done there.

This focus on excellence - of its employees and its work - can lead to the perception that the organization has the capacity to solve problems - all problems, including those related to diversity, equity, and inclusion.

I think my perception is NIST in general feels - and this is a cultural thing. I think the feeling is there's a lot of smart people here and we can figure stuff out. (INT:P117:M$50: 39)$

NIST's excellence, and its ability to "figure stuff out," implies that the organization is able to solve the problems it faces, even those related to diversity, equity, and inclusion. There is no need to go outside the organization to understand, address, or help solve organizational problems or issues.

In part, this focus on excellence is related to a culture where "science is king" as described by the interview participant below.

But again, we've got to hire leaders differently. And we have to be intentional. I actually had a conversation with somebody - it was a couple years ago now - that they were recruiting for a division chief position. And the first thing that my management said they wanted was somebody with a $\mathrm{PhD}$. And I remember laughing and saying, "Oh, did you want a $\mathrm{PhD}$ in organizational development or psychology or human resources or sociology, philosophy? You'll take any of those?" "No, no, no, no, no. We have to have a $\mathrm{PhD}$ fill in the blank hard science." And I said, "But why? This is a management position. This is a position--" "Well, they have to have the scientific chops to command the respect of the staff." And I said, "But what if the scientific chops don't come with good administration skills, good money management, good people skills, interested in developing others?" "Oh, that's okay. They can learn all of that." No, they can't. I've just decided flat out, if you're not interested in being a people manager, you shouldn't be in this job. And I can tell you, those conversations, we just play it out all the time. But it's, again, we want that science is king, which I think, from programmatic NIST perspective, that's right. We do science. And then, when it comes to the execution of that, we need to have people in roles that make sense. (INT:P105:F-1:03:18)

The quote highlights how scientific knowledge, background, and skills are more highly valued than knowledge, background, and skills in management or administration. NIST places more value on the science, even when the requirements and responsibilities of a position are not related to doing science, but rather to managing people. Quantitative and open-ended survey data also support this valuing of science, and of scientists. In S2Q7 of the survey, participants were asked to what degree they agreed or disagreed with the following statement: "I believe that NIST values scientists over other staff." Overall, $65.3 \%$ of respondents agreed or strongly agreed that NIST values scientists over other staff, and only $4.6 \%$ strongly disagreed with the statement. 


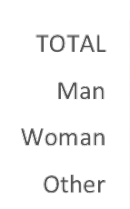

\begin{tabular}{|c|c|c|c|}
\hline \multicolumn{4}{|c|}{ I believe that NIST values scientists over other staff (N=1079) } \\
\hline $\mathbf{4 . 6 \%}$ & $\mathbf{3 0 . 1 \%}$ & $\mathbf{4 4 . 1 \%}$ & $\mathbf{2 1 . 2 \%}$ \\
\hline $\mathbf{4 . 9 \%}$ & $\mathbf{3 1 . 0 \%}$ & $47.4 \%$ & $16.8 \%$ \\
\hline $\mathbf{4 . 3} \%$ & $\mathbf{2 8 . 6 \%}$ & $38.0 \%$ & $29.2 \%$ \\
\hline & $23.1 \%$ & $38.5 \%$ & $38.5 \%$ \\
\hline
\end{tabular}

Figure 2: Value of Scientists

Graph shows the distribution of responses by gender where responses range from "Strongly Disagree" (left-most/dark gray) to "Strongly Agree" (right-most/dark purple). The number of respondents is shown in parenthesis.

Likewise, open-ended survey data from several different questions also highlight the value of scientists over other staff.

If I leave NIST, it will be because of the way the non-scientific staff are treated and the lack of respect from the scientists. There are many scientists at NIST who do not appreciate the non-scientific professions as just that -- professions with individuals who have worked in a field for extended periods of time and who have had training or what they do. Many feel as if they are better than we are at what we do, and treat us with a lack of respect. They feel their opinions on matters related to our work are more important than our professional expertise. (S6Q4:P1328:F)

NIST culture is very focused on the labs, which is understandable because that is the mission. However, a world class institute should value, prioritize, and acknowledge its support and administrative staff as well. While the scientific research is praised and awarded outside of NIST, the work that is done to support the research is not often recognized. (S2Q9:P1182:F)

At NIST, it is the science that is paramount, and scientists who matter the most. This seems especially important for two reasons. One is that women only make up $24.5 \%$ of the ZP (scientific/engineering) career path. If science and scientists are what is valued, and women are not located in those positions, they are less likely to be valued. Secondly, women predominate in the ZA (administrative staff) and ZS (administrative support staff) career paths. These employees provide essential support for the scientists in their work but are often not valued in the same way as those in the ZP staff, who are overwhelmingly men [1].

Another survey question provides insight as well into the ways in which science takes precedence, sometimes over the people who contribute to it. In S2Q6 participants responded to the statement: "I believe that projects are seen as more important than people." 


\begin{tabular}{|c|c|c|c|c|c|}
\hline \multirow{3}{*}{$\begin{array}{r}\text { TOTAL } \\
\text { Man }\end{array}$} & \multirow{2}{*}{\multicolumn{2}{|c|}{\begin{tabular}{l|l}
$8.7 \%$ & $50.1 \%$
\end{tabular}}} & & & \\
\hline & & & $32.5 \%$ & $8.7 \%$ & \\
\hline & $10.3 \%$ & $51.5 \%$ & $30.6 \%$ & $7.6 \%$ & \\
\hline Woman & $5.6 \%$ & $47.4 \%$ & $36.1 \%$ & $10.8 \%$ & \\
\hline Other & & $30.8 \%$ & & & $15.4 \%$ \\
\hline
\end{tabular}

Figure 3: Projects vs People

\begin{abstract}
Graph shows the distribution of responses by gender where responses range from "Strongly Disagree" (left-most/dark gray) to "Strongly Agree" (right-most/dark purple). The number of respondents is shown in parenthesis.
\end{abstract}

While the majority of respondents, both men and women, disagreed to some extent with the statement, $41.2 \%$ agreed or strongly agreed with it. While not the majority, it is telling that more than $40 \%$ of the employees who responded to this question felt that NIST values projects more than people. There was no significant difference both women and men equally agreed or strongly agree with the statement. This focus on projects over people is another example of the way science takes precedence, even over the people who contribute to it.

\title{
3.1.1.2. Objective and Rational: Visions of a Scientist
}

Objectivity and rationality are believed to be foundational elements of what it means to do science, as well as what it means to be a scientist. However, these characteristics are also associated more closely with men than with women [7], which provides a rationale for gendered assumptions about who is, and can be, a scientist. This is important given the value NIST places on science and scientists, as noted above.

Quantitative results show that most survey participants agree that NIST believes itself to be objective rather than subjective. In S2Q8 participants were asked to what degree they agreed or disagreed with the following statement: "I believe that NIST believes itself to be more objective than subjective."

\footnotetext{
TOTAL

Man

Woman

Other
}

NIST believes itself to be more objective than subjective ( $\mathrm{N}=1047$ )

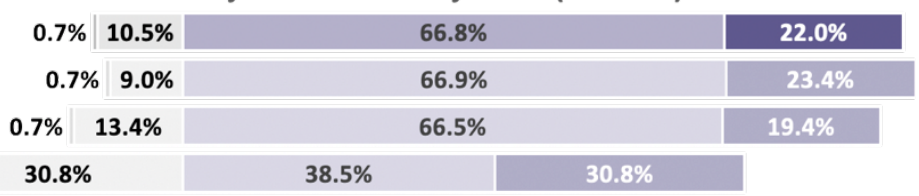

Figure 4: Objective vs Subjective

Graph shows the distribution of responses by gender where responses range from "Strongly Disagree" (left-most/dark gray) to "Strongly Agree" (right-most/dark purple). The number of respondents is shown in parenthesis. 
Overall, $88.8 \%$ of participants agreed or strongly agreed with the above statement. Again, there was no significant difference between the responses of men $(90.3 \%)$ and women $(85.9 \%)$. However, the large percentage of respondents who agree that NIST believes itself to be more objective than subjective highlights the way in which a culture of science dominates within the organization.

I LOVE these questions. Whoever wrote these gets a gold star. These are extremely indicative that someone is paying attention to the problems here. If I had to pick one question that sums up all the others: \#8 "NIST believes itself to be more objective than subjective" has hit the nail on the head. If we are an "objective" institution, it means that all people here succeed on merit. We can't be perpetuating systemic racism or sexism or homophobia if we are an *objective* agency; it merely indicates that those subgroups (women, LGBTQ+ folks, BIPOC) just objectively can't cut it, right? Otherwise they'd be succeeding too, right? Otherwise there'd be more of those people at NIST, right? Otherwise there'd be more of these subgroups in leadership, right? Of course this is garbage. It's been proven as such over and over again through biases in hiring, promotion, pay, work-life balance; even the heteronormativity embedded in our very badges and benefits and HR paperwork. But we cling to it. The notion that NIST is objective to the point where its policies and practices cannot be criticized or changed is outdated and needs to go. (S2Q9:P1454:F)

Seeing NIST as objective allows the organization and those who work for it to believe that biases, feelings, and preconceived ideas are not playing into decisions, into interactions with others, and into how and what the organization values. It can also lead to the assumption that as an objective organization NIST is as objective in the way it addresses diversity and inclusion as it is in the way it approaches its scientific mission.

One result of the focus on objectivity is that there is a perception of gender neutrality which works to maintain a status quo where men and masculinity are seen as the norm. It is difficult for women to be seen as scientists and as doing good science when "the cultural stereotype of the scientist as objective, rational, and single-minded is consistent with prescribed norms for men, but counter to stereotypes and prescribed norms for women" [7]. Both the interviews and the open-ended survey data highlight this link between objectivity and the norm of masculinity.

And so one day I was doing something in the lab. I was washing a graduated cylinder. And it kind of slipped and made a noise. It didn't break. It just slipped. And they looked up. And they laughed. And they said, "See? I told you. Women should be at home." In other words, they were saying that I have no place in science. ... And I was like, "That is not funny. (INT:P102:F-21:12 \& 22:59)

In my own small microcosm at NIST women are given less lab work and less technical responsibilities. They are given more administrative work. It is difficult to grow as a scientist, publish, and be "seen" as technically proficient when you have less opportunity to do work in the lab. Organizing lab cleanouts and parties for retiring staff are examples 
of responsibilities that fall almost exclusively to women in my group, these are time sucking holes that take time away from real work that matters for performance reviews. Having a management chain that is exclusively male perpetuates this type of behavior. (S2Q9:P2453:F)

A common thread in the data from this study shows how women in science are discounted and/or not taken seriously, how they are seen as peripheral to the science being done. This supports perceptions of women that we see in the culture at large - where women play a support role, there to care for and clean up after others.

These data describe a culture where women have less opportunity to be "seen" as scientists and as valuable members of the organization. As previously noted, women make up less than $25 \%$ of the ZP track, so they literally are not seen as much as men in the scientific ZP career path. While this is an increase from the $14 \%$ of women in the ZP career path in 1993, it is clear that their representation continues to be low [1].

Data from this study also indicate that value at NIST was not only about being a scientist, but also related to one's status as a federal employee versus an associate. Many of the openended survey responses spoke about how Associates are not respected and have fewer opportunities than federal employees.

People are respected for being federal employees. Associates are not respected at NIST. (S8Q2a:P2367:F)

Several of these responses also specifically noted that more women than men are Associates.

In my division a significantly larger percentage of women have been held as long-term associates as compared to men. (S2Q9:P1756:F)

The mere fact that there are a higher proportion of women in associate positions rather than staff positions shows that there is something wrong with hiring and retaining women at NIST. While some of this requires a societal change in culture, NIST could do more to attract women to NIST and retain them when they have children. The SURF and postdoc programs used to be targeted to women and minorities but that is no longer the case. These should be examined to see if they can be adjusted to attract more women and minorities. (S7Q14:P2128:F)

As indicated in the above responses, more women at NIST work as Associates and are more likely to remain in these positions longer than men who are Associates. This has long-term consequences for women's career status and salaries, in addition to the ways in which they are treated as Associates.

\subsubsection{Science as Competitive}

As a scientific organization, NIST values the work of its scientists, work that is often based on an assumption that it is done individually, by a scientist (most often a man) who works long hours, alone, to solve important scientific problems. This Principal Investigator model 
encourages individual work and competition, rewards individual work over teamwork, and values projects over people and relationships. Yet women tend to value the oppositeteamwork, collaboration, and mentoring [8]. These very different perspectives result in experiences and outcomes that provide advantages and opportunities for men while erecting barriers for women.

While many projects are worked on by teams of employees, questions about how NIST values and rewards this teamwork are important. Two questions in Section 2 of the survey focused specifically on teamwork - one asking about how it is valued and the other about how it is rewarded (Figure 5 and Figure 6 below). Interestingly, a far greater percentage of respondents agree that NIST values teamwork $(68.5 \%)$ more than it rewards teamwork $(54.6 \%)$. The differences between valuing versus rewarding teamwork are significant for both men (p-value (corr) $<0.001)$ and for women $(\mathrm{p}$-value $($ corr $)<0.001)$.

While there is no significant difference between the responses of men and women to these two questions, it is telling that a higher percentage of men $(70.2 \%)$ than women $(65.5 \%)$ agreed that teamwork was valued and rewarded (56.5\% of men agreed versus $51.2 \%$ of women). While not significant, these differences may highlight a different orientation to teamwork for men and women, something the literature supports [8].

$$
\begin{array}{r}
\text { TOTAL } \\
\text { Man } \\
\text { Woman } \\
\text { Other }
\end{array}
$$

$$
\text { (1) }
$$

r

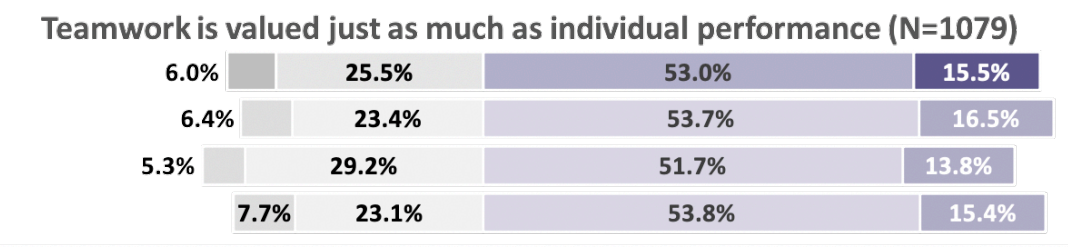

\section{Figure 5: Value of teamwork}

\begin{tabular}{|c|c|c|c|c|c|}
\hline & $8.5 \%$ & $36.9 \%$ & \multicolumn{2}{|c|}{$44.1 \%$} & $10.5 \%$ \\
\hline & $8.4 \%$ & $35.1 \%$ & \multicolumn{2}{|c|}{$45.0 \%$} & $11.5 \%$ \\
\hline & $8.6 \%$ & $40.3 \%$ & \multicolumn{2}{|c|}{$42.4 \%$} & $8.8 \%$ \\
\hline $7.7 \%$ & & & $23.1 \%$ & $15.4 \%$ & \\
\hline
\end{tabular}

Graph shows the distribution of responses by gender where responses range from "Strongly Disagree" (left-most/dark gray) to "Strongly Agree" (right-most/dark purple). The number of respondents is shown in parenthesis.

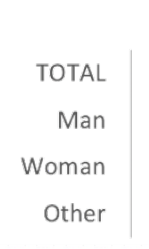

Figure 6: Reward for teamwork

Graph shows the distribution of responses by gender where responses range from "Strongly Disagree" (left-most/dark gray) to "Strongly Agree" (right-most/dark purple). The number of respondents is shown in parenthesis. 
This focus on value versus reward is important, since it communicates conflicting messages to employees. While they may hear that teamwork is valued, they know they will be evaluated and rewarded based on their individual work and contributions. When an organization says it values teamwork but does not reward it, employees may come to question how much it is actually valued. This was indicated clearly in interview data where participants spoke directly about how the reward structure (individual performance plans) discourage teamwork.

And I think actually that teamwork atmosphere is something that NIST is not good at motivating and propagating because we have these individual performance plans, which are really isolationist. (INT:P103:M-1:06:40)

Open-ended survey data also speak to this, especially responses from S2Q9 that asked if participants had any additional comments about NIST Culture that they wanted to share.

A lot of the performance incentives are aimed at rewarding individual contributions. It is required to be able to identify what an individual has contributed when it comes time to build a promotion package or even for the yearly performance appraisals. This disincentivizes - perhaps in subtle ways - teamwork. A true group effort may, in many cases, make it rather hard to clearly demarcate standalone contributions. (S2Q9:P2298:F)

There is a steep learning curve for younger, newer employees which makes it difficult to navigate the areas to pursue. There need to be more tutorials on things such as funding sources and leadership opportunities for new employees to help them come up to speed more quickly, especially when most start on a 2 year term. The learning curve makes it such that the newer voices are often not heard as clearly and have a difficult time identifying opportunities. Teamwork is so important, and I think it often does go overlooked. There is a lot of lab support needed to keep running our programs and to keep publishing results. On a division level it needs to be more recognized. (S2Q9:P:2389:F)

The focus on science as a competition where individual work is seen and rewarded was also identified by women in the interviews as a contributing factor to some of the discontent they felt because they wanted to work more collaboratively.

But there's no group involvement. It's not us all pushing forward. (INT:P005:F-5:35)

I want to be part of the team, and I've said almost these exact words, "I want to be part of the team because it gives me a sense of purpose about the work I do." (INT:P004:F4:09)

Research today argues that science organizations could benefit from changing the criteria for advancement to include common rather than just individual goals and promoting practices like mentoring in order to create a culture that values and rewards cooperation, collaboration, and creativity [9]. 
The competitive nature of the work at NIST was also shown in some of the open-ended responses. For example in S8Q2, respondents were asked: "Are there other reasons people are respected more at NIST?" An open-ended text box provided participants with a space to provide as much input as they wanted.

A disproportionate amount of attention and respect is given to people who are seen as the leaders of big projects or programs. This causes people to steal credit from others or to intrude and take over other the direction of other people's projects, to make themselves appear to have been the ones leading the project or program. I would like to point out that studies published in the social science journals on workplace bullying, show that most workplace bullying is done by men, and most of their targets are women. My personal experience is that NIST is no different in this. (S8Q2:P2271:F)

Number of papers published, amount of outside agency and internal competitive programs one gets funded, unfortunately, these are more valued than the development and deliverables of systems, chips, and new measurement techniques, which are harder to count than papers and money! (S8Q2:P2203:M)

While many open-ended responses note the competitive nature of NIST culture and its effects, a few open-ended survey responses did speak about how the NIST environment is not competitive.

I've given my "first impression" response to these questions, but I have to say I do not spend any time at all thinking about these matters. My work at NIST is not a competition, and I'm never moved to make comparisons to how I am valued or rewarded compared to others. I enjoy my work. I find satisfaction in completing it well. I am compensated well enough, and if I ever find that no longer true, I am free to leave. Asking these questions prompts people out of that mindset of work-focused satisfaction and into divisive comparison games. I think this is unwise. (S2Q9:P2477:M)

These negative case responses, while few, are important to recognize. Responses like the one above highlight the ways in which the culture of science presents a gender neutral view of the organization where all employees believe they are "free to leave" and believe they are "compensated well enough." However, the gendered nature of science, and of work at NIST, contribute to different beliefs about objectivity, competition, as well as to different experiences and outcomes for women.

\subsubsection{Dominated by Patriarchy}

The concept of patriarchy is central to the construction and enactment of gender inequality. Patriarchy can be defined as a social system where men hold power over women (and over some men), socially, politically, and economically. In a patriarchal system, men, and masculine ways of being and doing, are dominant, privileged, and valued more than others, even though on the surface the system seems neutral. Men have greater power to benefit from 
this system, although not all men benefit in the same ways or to the same extent. This creates a form of systemic bias against women.

The systemic bias created by patriarchy permeates the culture at large but is also foundational to the culture of science. In the realm of science, patriarchy has created and relied upon specific images and perceptions of what it means and who is best suited to be a scientist. For example, as early as elementary school, stereotypes exist that girls are not as good as boys in math and science and do not have a place in science [10]. Women and men internalize these stereotypes and research shows that women are aware of and adversely affected by it [11]. Examples of these stereotype are evident in both interview and open-ended survey data where women often described specific examples of them.

We were working on a project, and it wasn't automatic, so it needed to be done. And one of the gentlemen in the group assigned it to another person. And I was standing there looking, and he looked at me, and he said, "Well, you know that men are better at math." (INT:P116:F-19:04)

Often, these stereotypes are not recognized, and just seem like common sense. "Implicit bias is common, even among individuals who actively reject these stereotypes....(n)ot only are people more likely to associate math and science with men than with women, people often hold negative opinions of women in "masculine" positions, like scientists or engineers" [11, p. xvi]. These gendered perceptions, and the actions that result from them, often create barriers for women in the workplace - barriers that influence their opportunities and their outcomes.

One of these barriers is evident in the lack of women in the ZP career path at NIST, less than $25 \%$ as noted in the previous section. In addition, every year from 2011 to 2019, the proportion of women at pay band III (the lowest pay band considered in this analysis) is larger than it is for men, and the proportion of women at pay band V (the highest pay band) is lower than it is for men. Thus, more women are in pay band III and fewer in pay band V. In addition, women reach pay band IV in the ZP career path more slowly than their male counterparts at each level of education. The long-term effects of this are enormous, both financially and in terms of career status.

As discussed in the previous section, scientists are valued more than other staff and men are more likely to be seen as scientists than women. The same is true in relationship to leadership. More men are in positions of power and have the opportunity to move into leadership positions. An analysis of the data from human resources shows that even though there are more women in the administrative support (ZS) and administrative (ZA) roles, men have a greater chance of becoming a supervisor compared to women [1]. Not all positions have the same potential for career advancement which is evident in the ZS and ZA career paths. Currently, women still obtain fewer of the high-visibility, critical leadership positions which acts as a barrier to reaching the highest levels of leadership in the organization.

You sit in a meeting, you look around, you're like, "I'm the only woman. I'm the only technical woman. I'm the only manager woman." And we did that when we had the discussion about data. All the Deputies were there, and all the Division Chiefs were there, and all the office chiefs were there, and the OU Director's there, and the Deputy was 
there, and the AOA was there, and the SMA was there. So there were some women in the room. When you looked around you were like, "Of all the managers, there's 2 women and there's 16 men." (INT:P013:F-1:05:55)

In Section 4 of the survey, participants were provided with three statements specific to leadership at NIST and asked to what extent they agreed or disagreed with them. These are listed below, and results are shown in Figure 7 and Figure 8.

- "I would like to be in leadership at NIST" (S4Q7).

- "Women and men have equal opportunity to move into leadership positions at NIST" (S4Q8).

- "I believe I would be accepted and respected as a leader at NIST" (S4Q9).

There was no statistical difference of responses to "I would like to be in leadership" with over $59 \%$ of all participants saying they would like to be in leadership at NIST, with $56.7 \%$ of men agreeing and $63.0 \%$ of women agreeing. However, significantly more women $(52.5 \%)$ than men $(29.2 \%)$ disagreed with the statement about equal opportunity for women and men to move into leadership positions $\left(\chi^{2}(1)=58, p_{\text {corr }}<0.001\right)$. Thus, men and women both want to move into leadership, but participants believe that men and women do not have equal opportunity to do so.

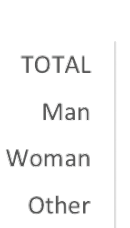

I would like to be in leadership at NIST (N=1065)
\begin{tabular}{|c|c|c|c|}
\hline $11.5 \%$ & $29.6 \%$ & $40.6 \%$ & $18.3 \%$ \\
\hline $12.2 \%$ & $31.1 \%$ & $39.1 \%$ & $17.6 \%$ \\
\hline $10.2 \%$ & $26.8 \%$ & $43.5 \%$ & $19.5 \%$ \\
\hline $16.7 \%$ & $25.0 \%$ & $41.7 \%$ & $16.7 \%$ \\
\hline
\end{tabular}

Figure 7: Desire to be in leadership

Graph shows the distribution of responses by gender where responses range from "Strongly Disagree" (left-most/dark gray) to "Strongly Agree" (right-most/dark purple). The number of respondents is shown in parenthesis.
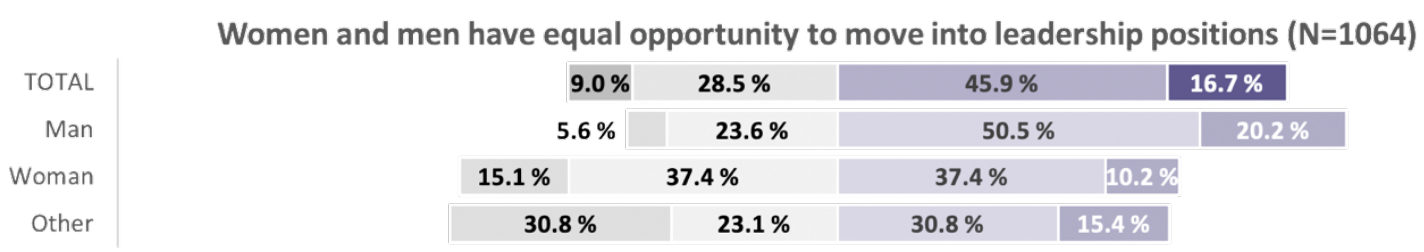

Figure 8: Opportunity to move into leadership

Graph shows the distribution of responses by gender where responses range from "Strongly Disagree" (left-most/dark gray) to "Strongly Agree" (right-most/dark purple). The number of respondents is shown in parenthesis. 
The lack of equal opportunity for women to move into leadership is certainly evident in the number of women currently in leadership at NIST, especially at the highest levels. The human resources data analyzed for this study show how few women have been able to attain leadership positions at NIST [1]. To date, NIST has had only one female Director out of 16. In 2019, there were a total of 471 supervisors at NIST; only $29 \%$ were women, while $71 \%$ were men. As of July 2020, only $21 \%$ of OU Directors are women, only $16 \%$ of Senior Executive Services (SES) are women, and only $5 \%$ of STs or NIST Fellows are women. Additional leadership positions include Division Chiefs where $37.5 \%$ are women and Group Leaders where $22 \%$ are women. Perhaps most telling is the NIST portrait gallery that recognizes the outstanding career contributions of distinguished scientists, engineers, and administrators to the work of NBS/NIST. Only $8 \%$ of these portraits are of women [1]. While $63.0 \%$ of women in the survey said they would like to be in leadership at NIST, it is unlikely that many of them will have the opportunity to do so.

The lack of women in leadership is also evident in the qualitative data, both from open-ended survey items as well as from the interview data.

It's not hard to see the disparities in leadership. Go to the 11th floor and try to find someone who isn't a white male in a leader position. I look around and out of the 2 division chiefs and 7 group leaders, there is one woman. The rest are white males. So telling me NIST believes in inclusivity and equity is out of step with reality. (S6Q4:P2130:F)

I don't think we'll see real progress on the inclusivity front until we have more equal representation in senior leadership positions (1 lab director is female, not too many female division chiefs, etc.). (S6Q4:P1330:F)

I would like to say that one of the core beliefs of NIST are to value men and women equally, but I do believe that this is not always reflected in promotions and leadership. (S7Q14:P2449:M)

Yeah. I mean-- well, look at our leadership team. All freaking white men. Look at NLB. I think there's 23 people in NLB. There's three women. (INT:P104:F-47:01)

The lack of representation is recognized by many employees, both men and women, some of whom believe that this shows a lack of value for diversity and inclusivity and runs contrary to one of NIST's core values.

This lack of representation in leadership also has both short and long-term consequences for women.

I have not spent enough time at NIST to understand first hand how promotions and opportunities for advancement look. However, based on second hand accounts and by looking at the demographic breakdown of the leadership at NIST, there is clearly something going on with the trajectory toward more advanced positions. I believe this has downstream effects (e.g., compensation). So, although for the same position, I believe a 
man and woman might be paid equally, because the woman is prevented from advancing, this means compensation is no longer fair. (S7Q14:P1344:F)

When women do not have equal opportunity to move into leadership, they do not have equal opportunity to advance their careers, increase their salaries, or learn additional skills.

In addition, a significantly larger percentage of women $(32.0 \%)$ than men $(11.4 \%)$ disagreed with the third statement, that if and when they did move into leadership, they would be accepted and respected as a leader-regardless of their gender, $\left(\chi^{2}(1)=68\right.$, $\left.p_{\text {corr }}<0.001\right)$.

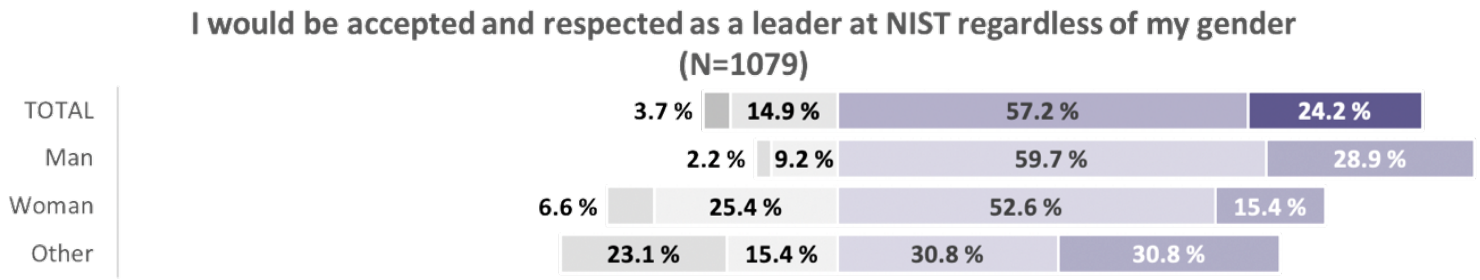

Figure 9: Leadership and gender

Graph shows the distribution of responses by gender where responses range from "Strongly Disagree" (left-most/dark gray) to "Strongly Agree" (right-most/dark purple). The number of respondents is shown in parenthesis.

Thus, women believe that while they would like to move into leadership, their ability to do so, and to be accepted and respected as leaders, is much less than for their male peers.

Open-ended survey data as well as interview data also demonstrate how employees, mostly women, believe they will not be accepted and respected as leaders.

I mean, I think this idea of-- a lot of people-- I don't know if it's problems. I would say it's characterizations, right? When you're a female leader, you get called bossy. You get called a bitch, right? Nobody ever calls a strong male leader bossy. They're authoritative, right? (INT:P113:F-40:25)

Men who raise their voices and complain are viewed as leaders and 'go-getters', whereas women who do the same are treated as agitators or 'loose-cannons'... I have personally experienced this at NIST, and externally. (S3Q12:P1610:F)

As noted in the qualitative data above, women in leadership positions are viewed in ways consistent with patriarchal norms and judged according to gendered assumptions about how men and women should act. Some survey participants noted that women and men are offered substantially different types of leadership opportunities that are consistent with gendered ideas about the type of work women should do. 
I observe within my OU that women at NIST are moved into "nurturing" positions of management, but not beyond to those that would make larger programmatic or technical decisions about the direction at NIST. For example, the women in my OU are project or group leaders but often are not in positions above that. (S6Q4:P1312:F)

Women get "leadership" roles in "outreach, service, and support" positions. No. Thank. You. (S6Q4:P2074:F)

Women should be "nurturing" and not be "go-getters." They should not be strong. This often positions women so they are not seen as effective leaders, which hampers their ability to move forward in substantive ways within the organization and continues to perpetuate these norms and assumptions.

The bottom line here is that NIST employees are disheartened at the way patriarchal norms continue to influence the organization and limit the potential of so many.

It's disheartening to work in an organization where the representation of women and underrepresented minorities does not reflect the external professional diversity for those communities. It's disheartening to work in an organization where one is told that because of their 'personality' they are not a good fit. It's disheartening to work in an organization, where the NIST leaders are not held responsible for NIST values - excellence, inclusivity, and perseverance, It's disheartening to work in an organization where OU directors explain the lack of diversity in a NIST focus area by attributing it to "women don't do quantum". This statement is not supported in the public literature. (S6Q4:P1426:F)

No one talks about gender and inclusivity issues at NIST. It seems to me that people are afraid of saying the wrong things. At this point, I don't feel that NIST is really committed to a diverse culture that actually works. It's more about appearances at this point, but maybe that will change. My group leader is a woman; she is one of the best managers I've ever known and would have made a great division chief and more. But 20-odd years later, she's still a group leader because she's been held down by a male-dominated organizational structure. I never wanted to be a part of NIST leadership because it seems like there's a certain "Kool-Aid" one has to drink. (S6Q4:P1447:M)

Many employees recognize these disparities and how they are connected to larger patriarchal norms and assumptions. They are disheartened and frustrated that these continue to influence NIST culture and create different experiences and outcomes for women and men.

\subsubsection{Rooted in Meritocracy}

Meritocratic ideology espouses that rewards are proportional to merit, where merit is viewed as ability and effort [12]. As scientists and engineers, we take pride in the objectivity and neutrality of our disciplines, and we believe we advance only those individuals with exemplary talent and dedication. We assume that those with the requisite training, experience, and drive will succeed in a meritocratic society, while those who do not succeed have only themselves to blame. 
The underlying premise of meritocracy is to hire, promote and compensate the "best" person for the job without lowering the bar or raising the bar for women. Yet many argue that the promise of meritocracy is a myth $[13,14]$ due to unconscious bias. The in-depth interviews found many examples to support the myth of meritocracy and how subjectivity and unconscious bias impacts hiring decisions, performance evaluations, and opportunities for advancement and promotion of women at NIST.

The survey further explored NIST federal employees' beliefs about meritocracy. Questions examined the roles of objectivity and subjectivity with respect to hiring, performance evaluations, access to opportunities for advancement, promotion and compensation. Nine survey questions explored characteristics of meritocracy.

\subsubsection{It's Who You Know}

The central belief of objectivity in meritocracy and the relationship of subjectivity in meritocracy was previously discussed in section 3.1.1.2 Objective and Rational: Visions of a Scientist. The belief in meritocracy minimizes the role of unconscious bias in decision making. According to Nobel laureate Daniel Kahmeman cognitive stereotypes help us organize our world into concepts that we rely on to make decisions [15]. Unfortunately without recognizing these unconscious cognitive stereotypes, women will be disproportionally impacted by hiring, performance evaluations and advancement opportunities.

The quantitative data show that women and men have statistically significant differences in their views of how opportunities are afforded at NIST. In Figure 10 more men (about $18 \%$ more) than women agree that "the best opportunities go to the most deserving employees" employees $\left(\chi^{2}(1)=35.0, p_{\text {corr }}<0.001\right.$ ). Similarly, more women (about $16 \%$ more) than men agree that "opportunities are given based on who you know" $\left(\chi^{2}(1)=29.8, p_{c o r r}<\right.$ 0.001) demonstrating consistency across the two questions.

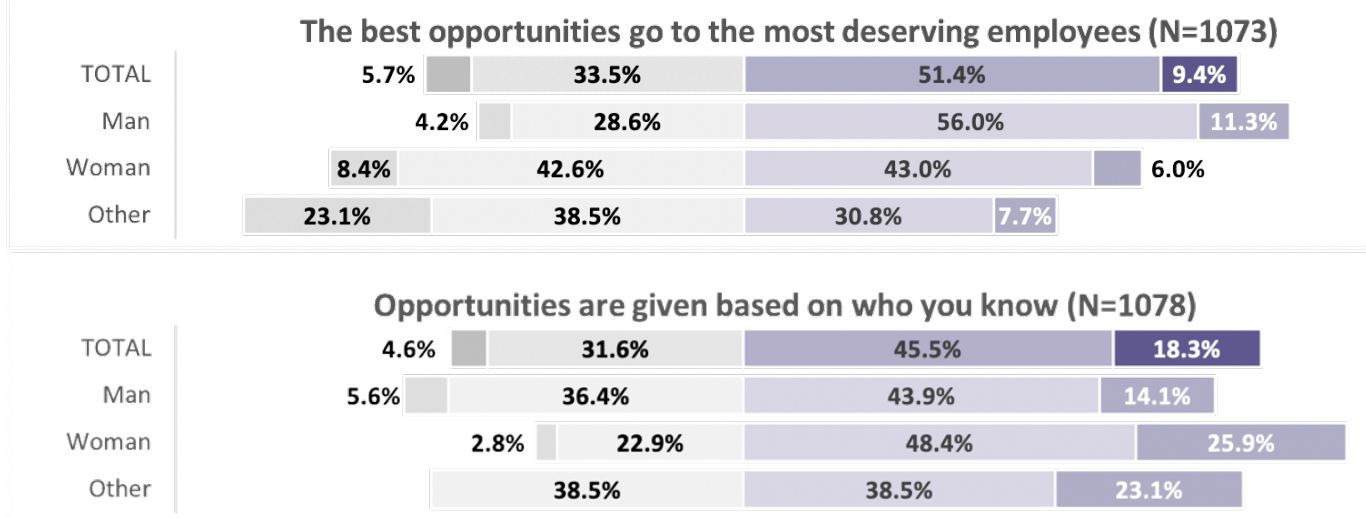

Figure 10: Meritocracy and Opportunities

Graphs show the distribution of responses by gender where responses range from "Strongly Disagree" (left-most/dark gray) to "Strongly Agree" (right-most/dark purple). The number of respondents for each question is shown in parenthesis. 
The responses to the open-ended question S2Q9: "Do you have any additional comments about NIST Culture that you would like to include?" elicited responses by both women and men that further elaborate on the perceptions of how opportunities are afforded. Many participants in both the interviews and open-ended survey responses referenced the dichotomy between actions and words supporting the notion that meritocracy is a myth.

The idea that people are promoted or rewarded for their good work, that is said verbally is fantastic but not rewarded in any other way, is very laughable. (S2Q1:P1469:F)

But there are certain categories of workers that seem to be-- they seem to have been left behind, let's say. .... They generally tend to be very loyal and assume that this is a meritocracy and that they will be rewarded for hard work. And that's not how the world works sadly.... they fall into this meritocracy trap. (INT:P117:M-11:10)

Rather than objectively promoting or rewarding good work, the in-depth interviews suggest that NIST runs on connections. Sometimes these connections simplify tasks and enhance solutions to problems both administrative and scientific. But sometimes these connections marginalize certain populations who are not as well connected. While the interview participants implied that connections (who you know) make a difference in hiring, opportunities, and promotions, the survey confirms the belief that connections make a significant difference in these three things. The open-ended survey respondents describe a broad range of connections and networking approaches used to offer opportunities from nepotism, to cronyism, trusted established pipelines, to loyalty, and other personal relationships rather than objective qualifications.

NIST culture is highly steeped in cronyism, and it's getting worse. There is no accountability when PPP are abused, so management can do whatever it wants to in personnel decisions. (S2Q9:P2046:M)

Well-paid positions i.e. the AOs people who got to the position majority of them have a connection. The AO in my area is a good example of this observation, her parent used to work for NIST, and several people she has connections to obtain her position are her parent's friends or family members. (S2Q9:P1364:F)

... that's kind of the backroom policy, backroom politics that I think can limit opportunity for people here and can ... be a recipe for limiting the amount of people that are on the team or the great fit as they say, right, because when you don't compete and you just name a successor as Group Leader or Division Chief or my role, what you have is kind of pressing the Easy button and not going through a process to determine who would be valuable based on what you're looking for. They are more or less picking personalities, and that is a concern of mine.... it becomes we select from the people we trust. And so there could be a lot of different reasons for why they have that, but I think that's definitely a thing that happens here. (INT:P103:M-11:40 \& 12:58) 
As these comments demonstrate, many believe established connections including family, trusted colleagues, "golfing buddies" (S2Q9:P1516:M), and the "cliques" (S2:Q9:P1546:M) afford opportunities. The interviews and the open-ended survey data report that connections are key to capitalizing on available opportunities. Yet, further exploration of connections in the survey discovered mixed messages. Connections were explored in several questions and across several characteristics including: the nature of the work, the perception of "fitting in" with teammates, and substantive conversations with colleagues and supervisors. For all of these questions, no statistically significant differences were found between the two primary genders implying that both men and women are connecting with colleagues and supervisors through conversations and both groups feel they "fit in" rather than being isolated by their work.

Figure 11 presents the survey data on conversations and interactions with colleagues and supervisors. As expected, the frequency of reported interactions decreases as the status of the subject of these interactions increases from over $90 \%$ reporting conversations with colleagues at least once a week or once a month, to about $88 \%$ for first line supervisors, and about $44 \%$ for second line supervisors to less than $20 \%$ with OU Directors. We found no statistically significant differences between genders for any of these questions. Thus, both men and women are interacting similarly with respect to frequency of conversations.

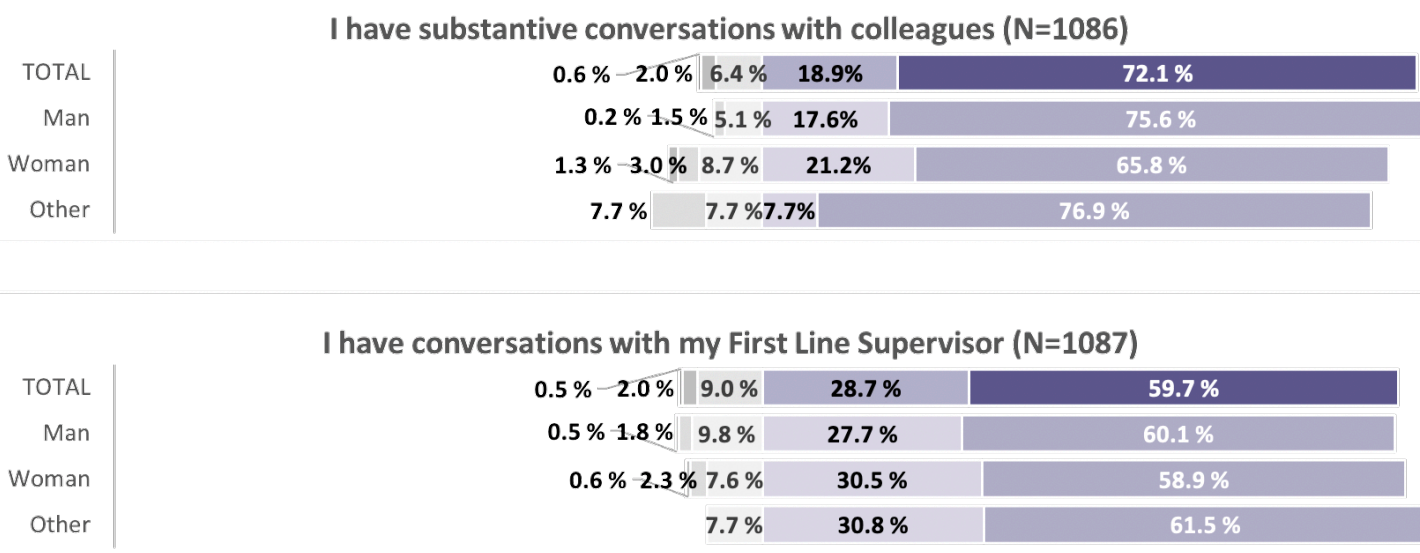

\begin{tabular}{|c|c|c|c|c|c|}
\hline TOTAL & $6.7 \%$ & $20.7 \%$ & $28.1 \%$ & $29.8 \%$ & $14.7 \%$ \\
\hline Man & $5.8 \%$ & $20.0 \%$ & $29.1 \%$ & $31.3 \%$ & $13.8 \%$ \\
\hline Woman & $8.4 \%$ & $21.9 \%$ & $26.2 \%$ & $27.2 \%$ & $16.2 \%$ \\
\hline Other & $30.8 \%$ & & $38.5 \%$ & $30.8 \%$ & \\
\hline
\end{tabular}




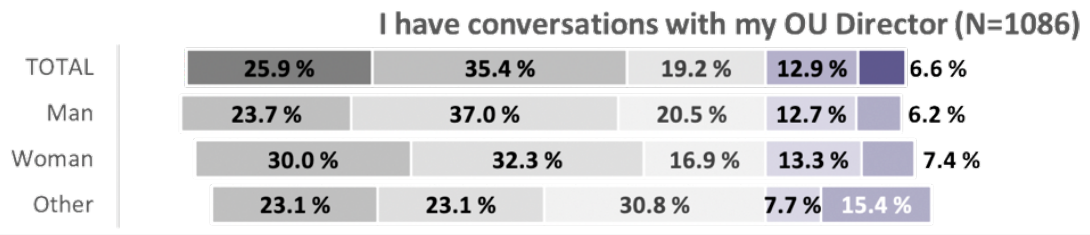

Figure 11: Frequency of Conversations

Graphs show the distribution of responses by gender where responses range from "Never" (left-most/dark gray, "Rarely" (once a year), "Occasionally" (at least once a quarter), "Sometimes" (at least once a month), to "Frequently" (at least once a week) (rightmost/dark purple). The number of respondents for each question is shown in parenthesis.

Similarly, we found no statistically significant differences when examining the nature of the respondents work and their perception of "fitting in" with their teammates as illustrated in Figure 12. Over a third of respondents report working mostly alone $(35.3 \%$ men, $34.0 \%$ women, $58.4 \%$ other gender). Despite the fact that over a third of staff report working mostly alone, over $82.0 \%$ of respondents, regardless of gender, report feeling that they "fit in" with the colleagues with whom they work.

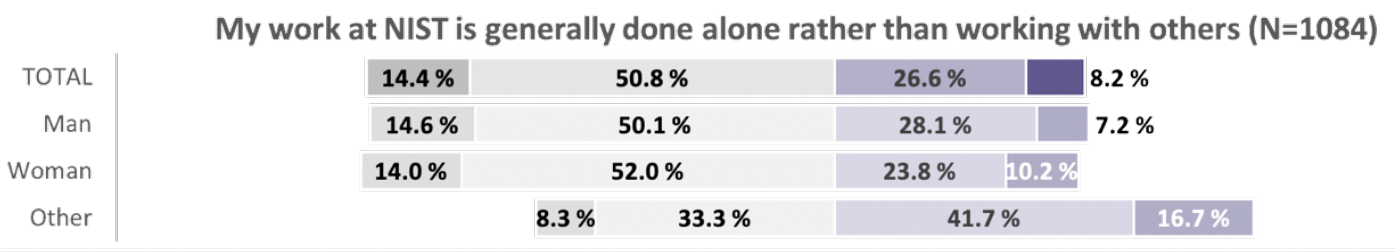

Overall, I feel I 'fit' in with colleagues I work with ( $\mathrm{N}=1082$ )

\begin{tabular}{r|} 
TOTAL \\
Man \\
Woman \\
Other
\end{tabular}

\begin{tabular}{|c|c|c|c|}
\hline $4.3 \%$ & $12.9 \%$ & $57.2 \%$ & $25.6 \%$ \\
\hline $4.8 \%$ & $11.2 \%$ & $55.3 \%$ & $28.7 \%$ \\
\hline $3.4 \%$ & $16.0 \%$ & $60.8 \%$ & $19.8 \%$ \\
\hline & $15.4 \%$ & $61.5 \%$ & $23.1 \%$ \\
\hline
\end{tabular}

Figure 12: Connections

Graphs show the distribution of responses by gender. The scale ranges from "Strongly disagree" (dark gray) to "Strongly Agree" (dark purple). The number of respondents for each question is shown in parentheses.

While the quantitative data identifies no statistically significant differences for the questions addressing connections, the interviews and the open-ended responses suggest that somehow the conversations and interactions are not sufficient to establish the connections and trusted network referred to by INT:P103:M. 
Regardless of where they came from, it's still a very academic-oriented mindset where you-- what have you done before in terms of papers, pubs, talks in a way that may not necessarily be completely in line with skill but it rewards the resume? ....- again, going back to the networking conversation, we do have the trusted network that we keep going back to all the time. And expanding our reach will be better there because a lot of it is, we expect to have the following result because we have a pipeline. .... I would say it's kind of tough to get into the pipeline, and we either have to make a conscious effort to go out to places we haven't gone, make a name for ourself, recruit, etc. And that's kind of that Easy button thing (INT:P103:M—40:50 \& 42:02)

The survey questions were unable to capture the nuances in the types of interactions and connections that develop or translate into "getting into the pipeline".

\subsubsection{Self-Advocacy: The Path to Connections}

While the survey suggests that conversations and interactions are not sufficient to establish the proper connections to afford opportunities, the survey data did identify self-promotion and self-advocacy as the key to building connections, networks, and becoming recognized. According to S2Q9:P1594:M "The squeaky wheel gets the oil." Despite few comments in the interviews about self-promotion, both genders had strong opinions about self-promotion in the survey responses.

Opportunity is not well distributed. The problem is not linked to the classic diversity areas of race, sex, sexual orientation/identity. ... NIST provides the best opportunities and advancement to narcissist, self-centered, braggarts. Complex team assignments performed for the benefit of NIST and the American people are not well recognized. (S2Q9:P2445:M)

I believe that self-promotion is the most-rewarded characteristic at NIST. Self-promoters are promoted to management while the most-competent are assigned more work by the less-competent management. And it's a vicious circle, with the incompetent promoting their incompetent buddies and heaping more work onto the hard workers who are left behind. ( S2Q9:P1177:M)

Unfortunately who you know and the need for strong and effective self-promotion marginalizes more women than men. Several survey respondents recognized how these practices disadvantage women.

As with all places of work, opportunities are based on skills AND on who you know. Scientists love to talk about a meritocracy and hold this up as a virtue of a work place. This is damaging to the inclusion of diverse view points. Diversity in a work force has been shown to be important to creativity and problem solving, and therefore is an important factor in building teams. A "meritocracy" view point can end up being damaging to building a diverse team. One reason for this: "being smart" is sometimes assigned to the loudest, most confident person in the room, which often pushes aside the 
skills of those who don't feel empowered to speak up. Oftentimes, due to their position or due to the socialization that society has imposed on them, women and minorities don't speak up. Sometimes this socialization even has to do with the group assembled- if there is only one young person, one Black person, one Asian person- can we expect them to be confident when faced with a group of older white people with strong opinions? Who will back them up? (S2Q9:P1312:F)

NIST culture is dominated by the internal competitive struggle to secure resources for oneself and one's staff to the distinct disadvantage of all others. NIST has even enshrined this cultural norm in the hallmark IMS proposal selection process; a process that asks our scientists to devote untold hours, days, and weeks to fashion themselves into advertising executives who must formulate a slick media campaign based on second-guessing the emotional biases of the management - a management that appears singularly skeptical and uninterested in the vast majority of what NIST scientists are working on, but are quite willing to suspend their disbelief in the face of a few well-timed pitches that favors buzzwords, catch-phrases, and sound-bites over substance, originality, and those very important and well-considered details that are considered too boring by those who manage us. As such: (1) opportunities go to those who sell themselves the best, not those who have a solid record of important findings and diligent effort. (2) Opportunities are given based on who you know, insofar as the persons you know have a better understanding of the emotional biases and prejudices that guide management decisions. (S2Q9:P2182:M)

Echoing the viewpoint of S2Q9:P1312:F that many women and minorities don't speak up, research shows that there is a substantial gender gap in self-promotion. Men engage in more self-promotion than women $[16,17]$. A female survey respondent described her struggle to advocate for her Band V and wondered if she were alone in her distaste of self-promotion.

The question about promotion is tricky. I am going through it right now, transitioning between and IV and a V. My boss asked me to write up my own package, and gave me an example of what a male colleague wrote. When I saw the example, I felt immediately inferior. HOW could I possibly make myself sound as awesome as my male colleague? Am I in fact inferior? Or do I just lack the ability to self-promote? I may never know. What I want to know is, how many women get to this transition point and then decide that they are not qualify or they find it too distasteful (to blow their own horn) to reach for that promotion? I am at that point right now - and I am desperately looking for a way out. (S6Q4:P1702:F)

The research shows the gender gap is not driven by a lack of self-confidence or by the drive of economic incentives but is the result of gender differences with respect to backlash, the idea that those women who self-promote are punished [16]. 
Irrespective, of how you established the connections whether self-promotion, cronyism, cliques, or old boys club, ultimately the data from the interviews and survey support an overall feeling from both women and men that "who you know" is the key.

the old rule "it's not what you know but who you know" is alive and well in our 2020 world. (S2Q9:P1233:M)

While recognizing the advantages and disadvantages of the principle of "who you know," several employees identified ways that the "old rule" could be addressed. Participants identified potential changes to practices and procedures including providing more transparency of the internal hiring process. Another participant believes that managers can play a pivotal role as champions for their staff.

Decision making, especially hiring, needs to be open, discussed by everyone, and justifiable. Currently decisions are made by mangers behind closed doors for political reasons. There are so many outstanding women and minority scientists, who have publication records as good or better than most NIST scientists, that we would recommend and recruit for NIST positions if we were only asked and if the opportunities were genuine. We currently have a job opening in our division, which is so vague and fixed, that I cannot recommend it to my colleagues. (S2Q9:P2090:M)

Success (as in likely any organization) is, in part, dependent on the presence of a 'champion' in management. So 'who you know' (how effective your management champions you) is an integral part of success." (S2Q9:P2586:M)

The role of champions especially mentors in advancing women's careers was discussed in both the interview data and the survey data.

... And I had a tremendous mentor in the leadership program that I actually started knowing before I even went into the NIST leadership program. And he threw a challenge at me and said, "If you're that bored, go talk to the associate director of management resources and see if he has any special projects he would like you to handle." And so I put a calendar invite on his calendar. (INTP001:F-5:47)

For this female interviewee, her mentor's challenge encouraged her to speak up and pursue additional projects and responsibilities that resulted in propelling her career forward. Given the positive experiences described by the interviews about mentoring relationships, several questions in the survey further explored the role of mentoring at NIST. The survey found that approximately $73 \%$ of men and women have mentors who help them navigate the environment and career paths. Most of the mentoring appears to be informal mentoring. Only about $19 \%$ of both genders report having formal mentors. Moreover, approximately $57 \%$ of men and women report being a mentor to others at NIST. Again, most of this is informal mentoring, only $13.6 \%$ of men and $9.7 \%$ of women reported participating as formal mentors. No statistically significant differences between genders were found for any of the mentoring questions. 
The open-ended responses also noted that formal mentoring opportunities were limited and most of the mentoring was informal. Concerns were expressed that the informal nature of mentoring could also disadvantage women.

With respect to mentoring. It would be very helpful if NIST had a broader mentoring program, rather than some, not well advertised, once a year opportunity. What's currently available is not very accessible to me, though I believe a colleague of mine has found it valuable. (S6Q4:P2254:M)

I have noticed that most of the men in my division are more comfortable mentoring other men than women and that is the result of almost all informal mentoring. (S6Q4:P1989:F)

Encouraging transparency and emphasizing the role of champions and mentors especially for the underrepresented populations at NIST may begin to change the culture from a who you know to a more merit-based or best-qualified approach.

The internal hiring process at NIST needs to change from "It is all in who you know" to

"Who is the best qualified" for the positions. (S2Q9:P1623:F)

The survey participants similar to the in-depth interview participants recognize that a belief in hard work is not enough to counter the effects of fewer connections, aggressive selfpromotion, and unconscious bias that disproportionally disadvantages women.

\subsubsection{Hiring and Promotion}

Both the HR data analysis and the in-depth interviews identified disparities in hiring and promotion of women consistent with STEM industry and academic trends [18]. With regard to hiring the HR data show that:

- NIST is consistently hiring more men than women for the ZP and ZT pay plans,

- NIST is hiring more women than men for the ZA and ZS pay plans, and

- for those categories of staff where we hire at least 20 new hires per year, the hiring percentages have not changed over the last nine years of analyzed data.

Yet when asked about equal opportunity to be hired, there is no significant difference between the survey responses for women and men. Both women and men agree that both genders have equal opportunity to be hired. While all agree that both women and men have equal opportunity to be hired, many survey respondents in the open-ended responses voiced concerns about the hiring practices especially as they impact NIST female associates. Concerns include the number of years women spend as Associates believing they will become NIST federal employees, fewer opportunities and stricter requirements for female Associates to become federal employees of NIST.

Another issue is that half of NIST are associates. Associates are treated differently from federal employees. To be blunt, associates are often treated as second-class citizens. They are denied many opportunities and "benefits" that federal employees have. (I don't been 
'benefits' as in health insurance etc. but things like being allowed to take training classes, being allowed to lead projects, even being allowed to go to conferences). And, my personal observation is that a disproportionate number of associates beyond the postdoctoral level of experience, are women. Thus, many of the ways that women at NIST are treated less equally than men, are explained away as not being because of their gender but because they are associates rather than federal employees. While it is true that male associates also receive this unequal treatment, it seems to me that more female than male scientists are being put into and kept in this "second class citizen" category.

(S7Q14:P2271:F)

That was the reason why I'd even stayed for a post-doc there was because they said there was a federal position, and I would be a shoo-in for it and had negotiated salary and everything for it. And it seemed like they were very supportive, and they were pushing me in this direction to stay on as a permanent member of NIST. ...(after seven plus years) This time around they happened to get more qualified people and that it would be unfair to offer me the job I had been working there for. (INT:P101:F-24:03)

Despite agreement that women and men in general have equal opportunity to be hired, there are significant differences for all of the questions regarding equal opportunities for advancement and promotion ( Figure 13 ). Approximately $20 \%$ more men than women believe that the opportunities for advancement are equal $\left(\chi^{2}(1)=52.1, p_{\text {corr }}<0.001\right)$, and that the promotion process is applied fairly regardless of gender $\left(\chi^{2}(1)=38.4, p_{\text {corr }}<\right.$ 0.001 . Furthermore, about $27 \%$ more men than women believe that time spent in a pay band is not influenced by gender $\left(\chi^{2}(1)=74.5, p_{\text {corr }}<0.001\right)$.

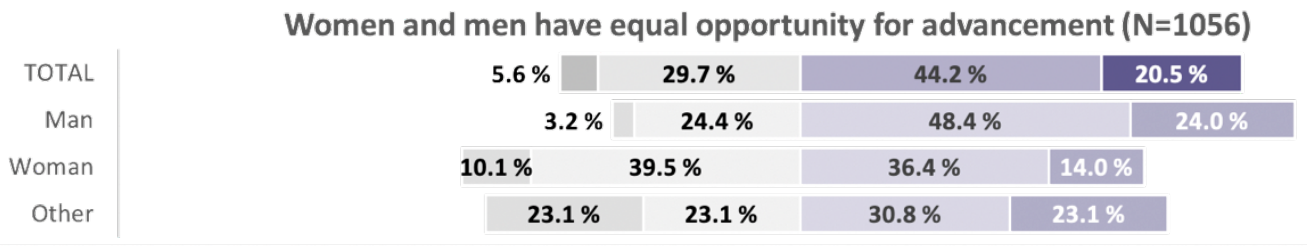

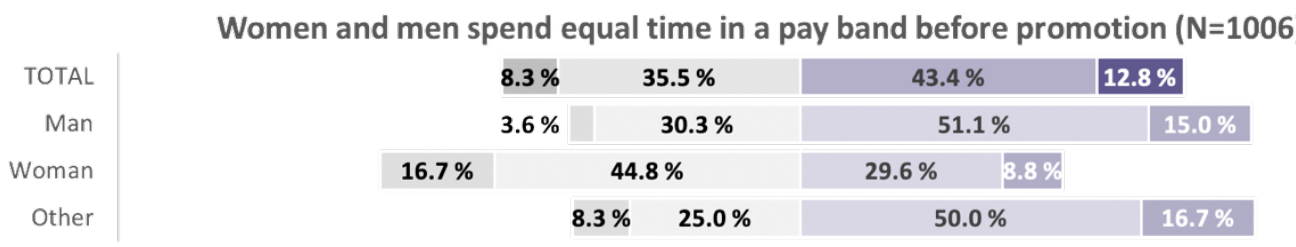




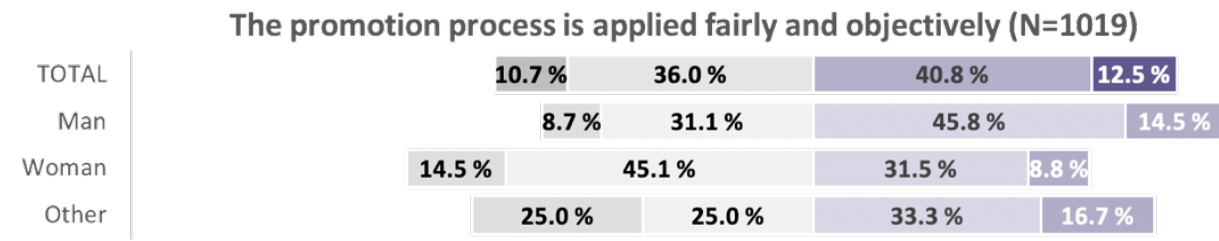

Figure 13: Opportunities for Advancement and Promotion

Graphs show the distribution of responses by gender. The scale ranges from "Strongly disagree" (dark gray) to "Strongly Agree" (dark purple). The number of respondents for each question is shown in parentheses.

The HR data confirms these perspectives. ZP women reach pay band IV more slowly than men at each level of education. ZA women with bachelor's or master's degrees reach pay band IV more slowly than ZA men with the same level of education. A smaller fraction of $\mathrm{ZP}$ women ultimately reach pay band $\mathrm{V}$ than $\mathrm{ZP}$ men. This difference is most pronounced among employees with a bachelor's as their highest completed degree. Throughout the first 20 years of her career in government, a female ZA or ZS employee is half as likely to hold a supervisory role as her male counterpart with the same years of experience in government.

A final question addressed compensation ( Figure 14.) Again, there was a significant difference between the responses of women and men. More men (27.4\%) than women believe that the compensation one receives is influenced by gender $\left(\chi^{2}(1)=79.7, p_{\text {corr }}<\right.$ $0.001)$. This is also confirmed by the HR data where salaries generally favor men in ZA, ZP, and Wage Grade (WG)) pay plans. Salaries for ZP employees appear to be trending toward gender parity, while salary differences between male and female ZA employees appear to be holding steady and differences for WG employees may be increasing.

$$
\begin{array}{r}
\text { TOTAL } \\
\text { Man } \\
\text { Woman } \\
\text { Other }
\end{array}
$$$$
\text { Compensation is fair for women and men ( } N=1009)
$$

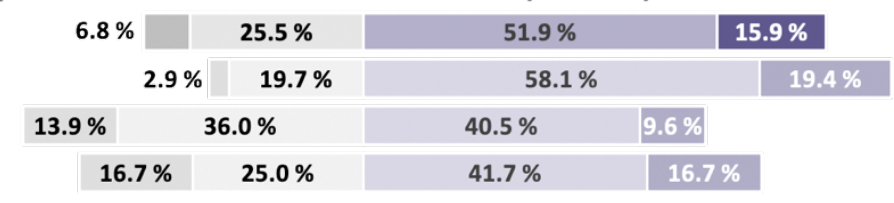

Figure 14: Views on Compensation

\begin{abstract}
Graph shows the distribution of responses by gender. The scale ranges from "Strongly disagree" (dark gray) to "Strongly Agree" (dark purple). The number of respondents is shown in parentheses.
\end{abstract}

The open-ended survey responses continued to elaborate on the disparities for advancement, promotion, and compensation. Predictably, respondents were passionate about promotions and advancement. Just as there are significant differences in the quantitative results based on gender, the responses also reveal very different perspectives based on gender. The women and many of the men who responded believe that the expectations for women are higher and 
women must be more qualified to be hired and work harder to achieve promotions and advance their careers much like the views expressed in the interview data.

I believe there is a clear statistical difference between women and men in ZP4, and 5 that cannot be attributed to the initial pool of ZP3's, so there are biases in the system (S7Q14:P2586:M)

Women have to work harder at the office/lab than does her male counterpart, to overcome bias that exists. Then she has to work harder at home with kids and running the home. The common saying is that a minority has to be twice as good as a white applicant to get hired or promoted. This is true for women as well. I have had experience with this over my career and at NIST. Those who cannot put in the extra time beyond the normal work week to get the work done to justify a promotion will stay where they are.

Supervisors reward this by giving raises and promotions to those who can do the extra work and spend the extra time working. Some simply cannot put in more than 40 hours. It's a problem. (S7Q14:P1201:M)

However, there was another group of men who believe that women are favored due to diversity initiatives even in the face of the HR data on promotions and numbers of women in leadership positions. One male respondent referred to NIST formal policies that discriminate against men. Others referred to a sort of "reverse bias" (S7Q14:P1826:M).

NIST has formal policies of actively discriminating against men in the selection process for several opportunities, for the purpose of increasing diversity. I believe that increasing diversity is not a justification for gender discrimination, and that these policies violate the civil rights of the candidates. (S7Q14:P1212:M)

I think it is easier for a woman to be hired, to be promoted, or to advance at NIST, than it is for a man. (S7Q14:P1546:M)

In the past, it appeared that there were disparities regarding equal pay and promotions for woman in favor of male counterparts. Currently, the opposite it appears to be true. There is greater push promote and advance females over equally or more qualified males. We should be evaluating the quality of work not the gender, race, etc of individual completing the work. (S7Q14:P1982:F)

Implying that women are promoted and provided opportunities based solely on gender, undermines women's qualifications, accomplishments, and leadership talents. Echoing the interview data, women continue to struggle with navigating promotion requirements. Women believe they have additional requirements than their male counterparts as P2523:F describes below. Many respondents believe overt or unconscious bias play a role. 
I have been given different reason what need to be done in order to get a promotion, such as write a paper, lead a project. but a male colleague that I know, he didn't have to do all that and got a promotion. (S7Q14:P2523:F)

On men/women having equal opportunities for advancement - again, managers tend to promote people that resonates with them. In a lot of cases, the people who are promoted are people just like them, i.e., male, white. So, no, I do not believe that women and men have the same likelihood of being promoted. (S7:Q14:P1702:F)

Here's why this is a problem. And I don't feel like the men have the same rules (INT:P013:F-4:08)

The survey respondents also highlighted the lack of transparency in hiring, promotion, and compensation further isolating women.

Hiring and promotion decisions are done in private and without transparency. (S7Q14:P1803:M)

When starting work, salary was negotiated but it should have been a clear option to give the women the SAME salary as men entering NIST at the same time with similar experience. Not pleased when it was discovered that there was a difference. (S7Q14:P1653:F)

After I became a NIST employee, I was very surprised and disappointed to find out that my salary was $\$ 20,000$ less than the salary of another peer who had been hired the same month as me with the same years of experience. And when I talked to my boss about it \{inaudible\} my Group Leader at the time, his response was, "Your husband has a good job. You don't need to make any more money." (INT:P113:F-6:13)

Overall, respondents reported more subjectivity in hiring, compensation, performance evaluations, advancement opportunities, and leadership opportunities for women compared to their male colleagues. Both datasets identified factors such as unconscious bias, the role of self-promotion, and connections as barriers for women. The barriers provide evidence that the paradox of meritocracy is a barrier for women at NIST.

\subsubsection{Role of Elitism}

Unfortunately, many management practices employed at NIST including merit pay, performance-based promotions, and ranking of employees by achievement are founded in elitist principles. As discussed in the previous sections, hierarchical social orders result from patriarchy and the culture of science, where scientists are valued more than other staff and men are more likely to be seen as scientists than women. More men are in positions of 
power and have opportunities to move into leadership positions at NIST. Patriarchy and the culture of science foster elitism and social hierarchies including age, gender, and race that govern staff perceptions and interactions and influence promotions and opportunities.

Hierarchical constructs including the role of age and length of tenure, which employees are most respected, and whose ideas are most influential were explored in the survey. The respect and value that NIST places on scientists over other staff was already discussed in section 3.1.3 Based in Science.

Who has influence? Who do we listen to at NIST? What is the relationship of tenure and influence? Staff who have been around a long time are listened to more than staff who have been at NIST for a shorter period of time (S2Q3). Both women $(56.4 \%)$ and men (63.9\%) believe that staff who have been at NIST for a long time are listened to more than staff who have been a NIST for a shorter period of time. There was no significant difference in the responses between women and men. Interestingly, this seems to contradict that the majority of respondents $(87.8 \%)$, regardless of gender, agree that people they work with listen to their ideas when making decisions (S4Q4). Again, no significant difference. However, the openended responses provide a more nuanced perspective.

The open-ended response comments reveal how the belief in tenure has been overemphasized and disadvantages younger staff and even staff who have established careers but are new to NIST.

The biggest divide I've noticed is between older staff and younger staff. NIST has a huge inertia and part of this is the desire of older staff to do things "the way it's always been done." I have personally been told "no" about a reasonable suggestion without any qualification, or any alternative approach to get at a similar result or a more feasible result. My management has also stated explicitly that saying no to staff ideas often is healthy for the organization. I understand this, but it's rife with danger on the inclusivity/gender front -- whose ideas get shot down more often? I would prefer to see a culture where all ideas are taken seriously and management works with staff to see how an idea can be implemented within organizational constraints. (S6Q4:P2009:M)

Others were frustrated by how long you had to be at NIST before you were recognized as "experienced" and your voice would be heard.

One thing that really does not sit well is the use of the phrase "Oh, so you're just a baby then" when you arrive at NIST. That is a very odd thing to say to a grown adult who is accomplished in their field. This is meant to indicate that you are young at NIST and "don't get a nod" of recognition until you are 20 years in. That is ridiculous. How can we address this as a community to reverse this common culture? That means that new hires don't have a voice for 20 years...?? (S2Q9:P2451:F)

I feel like I am constantly having to work past the perception that I'm a young female without enough real world experience. I am 40 years old. NIST culture warps our 
perceptions of how long it takes to advance in a career. Age matters. Time at NIST matters. Gender is also a factor, because -- coupled with age -- it makes those other things matter more. All this, while the rest of the world's leaders are younger and advancing faster. It certainly puts NIST at a disadvantage for keeping and promoting talent in house. (S2Q4:P1926:F)

Much like P1926:F many expressed concern that overemphasizing tenured staff limits new ideas and thoughts disadvantaging and impeding new opportunities and growth both for individuals and the organization as a whole. Thus many emphasized the need to listen to newer and younger staff while recognizing the value of "seasoned staff ."

... seniority has tremendous value, however I continue to see the same seasoned staff brought in on high-level efforts (e.g., strategic planning). New thoughts and thinking is what will propel this organization forward. We need to find ways to balance the thoughts of the seasoned staff to those that can bring so much that have only been here their entire career. (S2Q9:P1591:F)

I believe people that have been at NIST for longer periods of time have established themselves as knowledgeable in certain areas versus 'new' people. I do believe the ideas and thoughts of newbies are considered and not just thrown aside. (S2Q9:P1387:M)

And empowering some of the younger staff too to be able to jump in and-- because age does not matter in abilities. And you can have somebody sort of younger, but also not necessarily inside your ring. That outsider perspective can bring a lot of value. So case in point, the strategic planning effort. When I looked around the room, when they first kicked it off, I was like, "These are not the people you need in the room." There were some of us. But there were division chiefs and such. I'm like, "These are not where you're making changes." Or some of the old-- where they were kind of joking with each other of, "Oh. We've been through this before," sort of thing. You're not going to make changes either. Don't bring in your postdoc who's 60 years old. That's not who's going to bring things forward for the organization. (INT:P109:F-53:40)

Who is heard and listened to significantly impacts career opportunities and advancement at NIST. Elitism and connections influence which voices are heard disadvantaging women.

While many of the comments addressed the two survey questions directly, many respondents took the opportunity to express additional concerns about elitism and described a hierarchical social status that they believe dominates the NIST culture. Over 175 open-ended responses focused on the theme of elitism substantiating and enhancing the in-depth interviews perspectives.

Several respondents in the survey were quite unequivocal in their characterization of NIST's culture of elitism using terms such as "caste system" (S2Q9:P1432:M) and "class structure" (S2Q9:P1926:F) much like the "second class citizen" (INT:P104:F-7:08 \& 7:59) quote from the interview data. Others recognized the relationship between "social and economic" castes and gender and ethnicity. 
There is a caste system at NIST. Scientists at the top, associates and admin staff in the middle, and then support staff. (S2Q9:P1432:M)

I feel like working in operations, NIST is set up with a bit of a second-class citizen type view. ... And so, you're going to-- I always tell people, "You're going to need-- expect that you're going to hear that from some people, and it's something that you're going to have to deal with. I think that is part of our culture." (INT:P104:F-7:08 \& 7:59)

Silos and (cultural) bubbles encourage us/them attitudes that originate in the broader society. E.g., white collar/blue collar, scientist/plant/finance, staff/management, worker/bureaucrat. Those social/economic castes are strongly coupled with ethnicity and gender. (S2Q9:P1813:M)

The "caste system" sets the stage for bias and for valuing particular staff above others. Examples included valuing scientists and PhDs over administrative staff or federal employees above Associates.

NIST has long valued scientists over others. It is a running joke among the lay persons. Even some honest scientists will admit the bias. NIST also suffers from $\mathrm{PhD}$-itis. That is, they are quite arrogant when it comes to knowledge. This leads to elitism and a condescending attitude toward non-PhDs. Again, some honest scientists will admit this bias...but claim professors are worse! (S2Q9:P1931:M)

I found over the years that lack of inclusivity at NIST was very often related more to educational level or payband and job positions than gender/race identities. E.g., a $\mathrm{PhD}$ is BETTER THAN an MS; a PhD in Physics is BETTER THAN Chemistry; a ZP is BETTER THAN a ZA or ZT ... (S1Q5:1295:F)

The bad is it's very monoculture. It's a lot of PhDs. And there's a little bit of elitism in that where, "You're not a PhD? Oh.".... So sometimes you get that, "Oh, I'm a PhD." I'm like, "Nice for you. But you're still calling me for help because you don't know how to do this," so. (INT:P013:F-15:43)

Administrative staff are treated secondary to scientific staff. (S2Q9:P2333:F)

I think there is a huge difference between a federal employee and a guest researcher. I think guest researchers are kind of exploited. .... If NIST can't set a balance between Federals and Guest Researchers, the guest researchers will not feel sense of connection and belonging. (S2Q9:P2363:M)

Preferences for degrees and valuing certain types of staff over others prohibits inclusivity. The desire for excellence continues to promote an overall atmosphere of elitism as demonstrated by the following comments.

At times it is hard to determine if the issues I encounter stem from gender/inclusivity issues or from scientific snobbery. My career path is technical but not scientific. I do not 
do research or publish but have a technical role in my OU. My lack of scientific credentials has been called out on various projects by all levels of colleagues (co-workers to managers to OU Directors). I even had a colleague imply that I did not have the mental capability to understand a text written at a 5 th grade level. I am not sure if it was because of my gender, because I did not have a PhD, or both. I do know that I have had to work very hard to demonstrate competency in order to be trusted to do my job. (S6Q4:P1658:F)

Without the scientists there is no need for other staff. I am from ADMR and I understand we have an important role to play in partnering with ADLP to achieve the NIST mission. But at the end of the day, the jobs we do are readily fillable by others whereas the same cannot be said about researchers. (S2Q9:P2331:M)

Elitist views like these encourage hiring from a few select universities. Likewise, these views limit opportunities for advancement and leadership roles for women.

NIST culture is elitist and need to start hiring from universities around the country. NIST should care that their population doesn't reflect the US population. Why is the upper management almost all white males, with a recent female hired? (S2Q9:P2500:F)

NIST knows how to do science. NIST does not know how to lead and manage and support science. While there is training, it is not effective training. Managers and leaders are selected based on scientific skills, not management and leadership skills. NIST does not effectively develop these scientists into effective leaders. NIST then loses a good scientist and gains a poor manager. They are different skill sets. (S2Q9:P1226:M)

And I will tell you, I mean, I work in an administrative field. So I then have sort of two strikes against me at NIST, right? .... then, being a woman-- and I will also say, I am on the younger side. I became (a supervisor) in my late 30s. And closer to middle 30s, actually. And so, I was young. And I'm a woman. And that, I think, was--. So, I mean, there's just all kinds of Xs across there. (INT: P105:F-19:08)

The "caste system" and the resulting bias also enable the tolerance of "bad behavior" by those in the top tier of the class structure, white men as the following quotes exemplify.

NIST is tolerant of unprofessional behavior, especially from respected white male scientists. (S2Q9:P2348:M)

It's not only about the berated. It's about the berater also. It has to be about the aggressor and the target. And we have to do better. But for so many years, it was always about that person because, "I'd worked with that person for the past 30 years, and they just have really high standards," or, "They just work so hard. Their job is so important to who they are and their identity that when people fall short, they just snap." Well, just snapping isn't acceptable, right? But again, there was this concern. And again, in most cases, the people who would be in charge of the accountability for that berater couldn't get over the relationship. (INT:P105:F-59:38) 
I see a continued pattern of bringing in "experts" and "others" to address long known internal issues with poor management and leadership. There is a stunning lack of accountability for managers who do not manage effectively. I see this pattern getting ready to repeat with plans to hire a diversity coordinator - diversity will become the coordinator's issue to address and managers who do not promote or engaged effectively around diversity issues will again be allowed to coast. (S2Q9:P1330:F)

The lack of accountability, the explicit and implicit biases of elitism affect all of the staff at NIST, but the culture of elitism further marginalizes women and minorities.

The final question of the survey examined which characteristics afforded the most respect at NIST. This question asked respondents to pick their top three reasons.

S8Q2: At NIST, people are respected more for the following:

For being a scientist

For being a man

For having been at NIST a long time

For the kind of degree a person has

For getting things done using administrative expertise

For the job description a person has

For the number of awards a person has

For the way a person dresses

For the way a person speaks

Among the three top categories were "being a scientist,", "for having been at NIST a long time" and "for the kind of degree a person has," followed closely by awards.

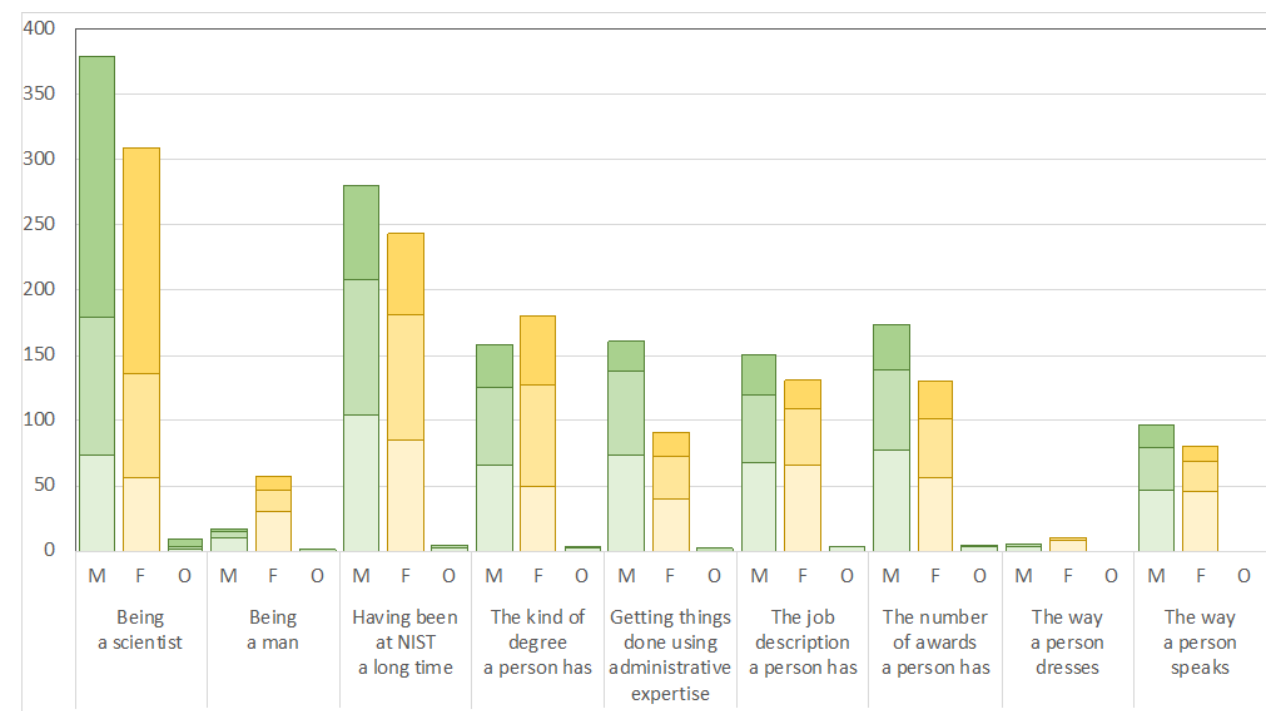

Figure 15. Respect and Recognition at NIST

For each question, the responses are divided by gender ( $\mathrm{M}$ denoted male, $\mathrm{F}-$ female, and $\mathrm{O}$ - other gender). The intensity of color represents the rank order, with the darkest color corresponding to rank 1. 
The perspectives of staff on respect and recognition presented in Figure 15 are consistent with and confirm the interview data. They also confirm the other survey questions on elitism with respect to degrees and tenure. According to many participants in the survey and interviews, the focus on NIST's scientific excellence has undermined the significant role and value of the work of many at NIST.

I've been at NIST for over 10 years and in that time, I continue to see language and rhetoric that we have the "best scientists" in the world. That's absolutely true, however that messaging undermines that immensely valuable foundation that ENABLES the scientists to do their work. From day one of orientation, the focus was on the \# of staff that had phDs. Yes, we are a science organization but we need a more inclusive way to celebrate all staff at NIST. We all work toward the same mission here - it would be nice to highlight that. (S2Q9:P1591:F)

I feel it's important to note that this may not be done intentionally and I understand the importance of the science performed at NIST. However, since beginning my employment at NIST, nearly 15 years ago, I can think of almost no NIST-wide events that sought to bring the science side of the house together with the support side of the house for camaraderie or to create a sense of unity - like an annual NIST Day or something to that effect. Annual award ceremonies and town hall meetings do not count in this regard because they are not designed for that purpose. I can't think of a time a NIST Director, AD, or Lab OU Director attended an annual financial management conference, HR event or the like if only to make opening remarks or to thank staff in these areas for their contributions. (S2Q9:P2265:F)

Many employees recognize the disparities and lack of respect resulting from elitism and the unspoken "caste" system at NIST and how this bias tends to disproportionately disenfranchise women and minorities.

\subsection{Gendered Experiences}

Th previous sections describe how NIST's culture is shaped by beliefs in scientific excellence, patriarchy, meritocracy, and elitism. These strong beliefs result in an environment where women and men experience NIST quite differently. The data presented in this report demonstrate how the ideologies that underpin the NIST culture act as facilitators for men and barriers for women. These ideologies also perpetuate social and cultural biases and unconscious biases that manifest as micro-inequities and microaggressions further contributing to different experiences for women and men, further reinforcing stereotypes, erecting barriers and disadvantaging women at NIST.

The in-depth interviews identified many gendered experiences at NIST including obvious harassment and unwanted physical attention to more obscure microaggressions such as subtle 
put downs, questioning competence, and the mommy track. The most common forms of gendered harassment experienced by women at NIST today are the microaggressions depicted below the surface of the iceberg in Figure 16.

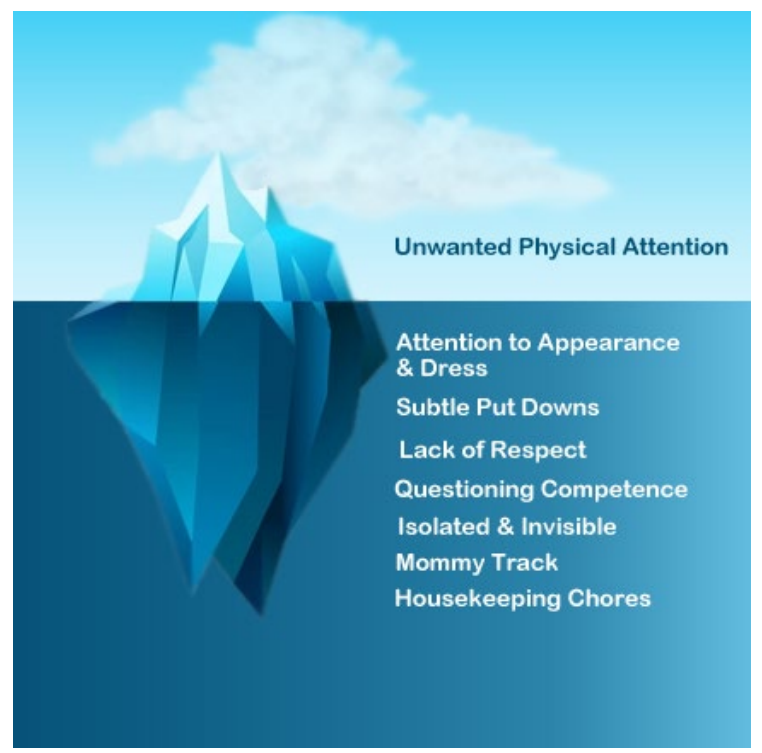

Figure 16: Microaggressions Experienced at NIST

\subsubsection{Harassment and Physical Attention}

Unfortunately, NIST is not immune to harassment, several female interview participants described instances of unwanted sexual advances, of touching, and of invasion of their physical space.

We were on a work trip, and she's like, "I want to tell you about what's going on here, and I would like this to be communicated up the chain, but not formally." ... So, what happened was she was-- so one of her Group Leaders was basically just making sexual advances to her all the time. ... It was crazy. She finally complained after years of enduring it, right? And then she decided to complain when another girl came to her and said, "This has been happening to me, too."(INT:P008:F_ 42:09 \& 42:29)

"I've been tracking how you inappropriately touch me, and it will stop now. You will not touch me again." He used to rub my hair. He would rub my shoulders. He would rub my upper back. He would rub my lower back when I would walk through doors. Incidentally, everyone knew that he did this, because he was a "Southern gentleman" and that's, wait for it, just how he is. And it was absolutely excused by everyone at the time.

(INT:P105:F-12:24) 
While more women experience harassment than men Figure 17, a male survey participant also described his experience with harassment.

My own experience with harassment involved an uncomfortable level of touching, as well as what I felt was relatively benign (albeit somewhat uncomfortable) commentary about how I dressed. (S3Q12:P1768:M)

Two questions in the survey focused specifically on harassment-one asking if the participant had experienced harassment and the other if they were aware of others experiencing harassment.

- S3Q10:I have experienced harassment because of my gender (for example: inappropriate touching, comments about the way you dress, comments about body parts, etc.).

- S3Q11:I am aware of others who have experienced harassment because of their gender (for example: inappropriate touching, comments about the way you dress, comments about body parts, etc.).

For these two questions, participants were provided with three response options:

Yes, has happened more than once; Yes, has happened at least once; No, has not happened.

All genders (approximately $40 \%$ of women and $30 \%$ of men) were aware of others being harassed. Significantly more women (approximately $26 \%$ ) than men (approximately 3\%) report experiencing harassment $\left(\chi^{2}(1)=119, p_{\text {corr }}<0.001\right)$. Those who responded "Yes" were asked a follow up question "Did you report the harassment?" and, if so, whether the issue was resolved to their satisfaction. Only, $26.1 \%$ of the women who experienced harassment at least once reported the issue. Of those who did report the harassment, about $30 \%$ said that the issue was resolved to their satisfaction while another $20 \%$ of women mentioned they are not aware of the resolution or the result of their filing. Of the $3.2 \%$ of men who experienced harassment due to their gender, about $15 \%$ reported the harassment, and all of them reported that the issue was not resolved to their satisfaction.

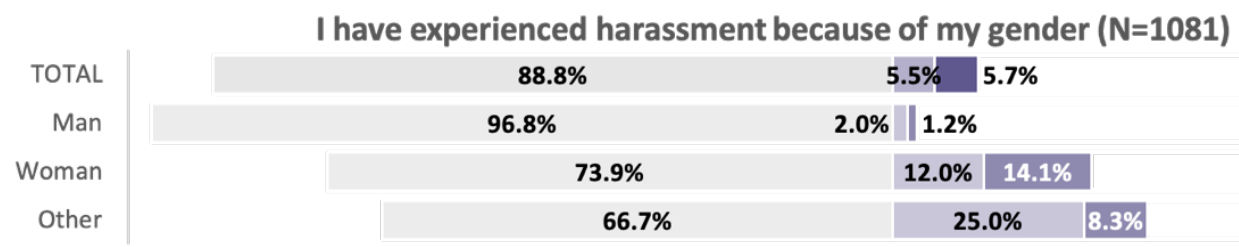

Figure 17: Percentages of those Experiencing Harassment

The graph shows the distribution by gender where responses range from No, has not happened (left most /gray), Yes, has happened at least once, to Yes, has happened more than once (right most/dark purple. The number of respondents is shown in parentheses. 


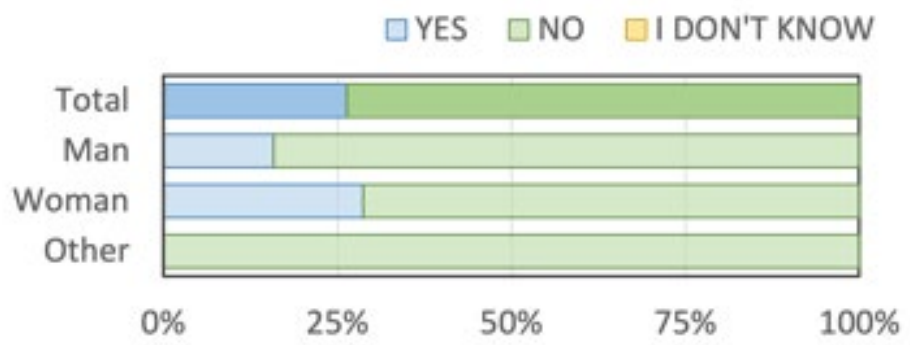

Figure 18: Reporting Harassment

The survey data support the interview data where women described incidents of harassment, their reluctance to report harassment, and their frustration with the process and the lack of resolution. Comments to the open-ended text box S3Q12: "Do you have any additional comments about your experiences at NIST based on gender that you would like to include?" expressed concerns about possible repercussions echoing the interviews. It's a "hidden culture" that happens "behind closed doors." Women do not report because it is not safe to do so. Thus, any numbers related to sexual harassment must be questioned as being low [19].

I think it is sometimes the things you don't hear about that are of concern. I suspect that most sexual harassment happens behind closed doors and that it is difficult for women to report this without fear of retaliation. (S3Q12:P1759:M)

The way in which harassment is addressed and "resolved" at NIST makes not reporting it the safer way to handle situations. I know multiple women who have been harassed.

VERY few ever report it. The process is designed in a way that the repercussions come back on the woman. The man (or woman) may be "relocated" or meetings to discuss the situation are had, but the problem is that the men do not have consequences for their actions so why would they ever change. If you want to fix this hidden culture that is very much prevalent across campus, then you need a way to allow the safe reporting (a lot of times this means not involving the other person) and the situation needs to be corrected, not just patched. (S3Q12:P2130:F)

NIST leaders are reluctant to address misconduct and harassment by those they deem at a high enough level that they should be treated differently. "Successful scientists", Fellows, OU leaders, and others have behaved inappropriately but have not been appropriately admonished and reprimanded as often as a zero tolerance type of policy would suggest. The outcome is fear of reprisal to challenging the powerful, which leads to less than optimal culture. (S3Q12:P2163:M)

Based on my experience, I feel that inappropriate behavior of men towards women at NIST have mostly been ignored when reported to management and HR. They (management and HR) appear to not acknowledge systematic behavior of certain 
offenders, but try to over simplify the situation as individual conflicts between a pair of people who happen to be of opposite sex/gender. (S3Q12:P2139:F)

Despite discrimination law and the opportunity to file a formal complaint, physical harassment is still a threat women must navigate at NIST. Women must also navigate how they dress. The interviews found that women were quite aware about how they dressed, both to avoid attention and to be taken seriously.

Early in my career, it had an impact on how I dressed, and the thought that I put into it, where I was very careful to make sure that I wasn't wearing anything-- my husband even jokes, how I acted and how I dressed in my 20s was much more reserved. And I said, "Because I needed to at that point." I needed people to see me for my abilities and nothing else. ..... But I feel like I've got enough now under my belt that I don't have to worry as much of wearing my boots to go to meetings. Or wearing something a little more form-fitting, and not thinking, "Oh. She doesn't know what she's--" There's no way. You know what I mean? I have consciously thought of that my entire career, of how I look and what I need to-- "Maybe I shouldn't wear that to that meeting. That's probably a little more-- I probably shouldn't do that." Or the click-clack of my shoes down the hallway... (INTP109:F-41:34 \& 43:31)

Given, these concerns a question addressing physical appearance and capabilities was also included in the survey.

- S4Q3:I believe that the way I dress influences others' view of my competence.

Four response options were provided: Strongly Disagree, Disagree, Agree, Strongly Agree.

There is no significant difference in the responses to this question, $48.9 \%$ of men and $56.4 \%$ of women agree that the way they dress influences how their competence is judged. Among the respondents for other gender, about $75 \%$ agree with this statement. However, the comments in the open-ended text box again confirm the interview responses by describing how appearance and dress disadvantages women.

Regarding dress, as a woman scientist, it is essential that I DRESS DOWN so that I don't get mistaken for a secretary. Of course on the phone I'm still mistaken as a secretary but what can you do? So I dress like a slob and then it's hard to be taken seriously. There is a sweet spot in there, so this isn't an unsolvable problem, but it is one little micro-issue that adds up when you integrate over a whole career. Men don't have this problem. (S6Q4:P1175:F)

That when people treat me in a certain way at work they treat me that way because of what I can do about my job, my expertise, they want my opinion, not because of what I look like, or how old I am, or how tall I am, or what clothes I wear, how much makeup I wear, my hair. It's what can I do? Why am I here? (INT:P013:F-40:23) 
The women in both datasets expressed a desire to be judged on their abilities and not their looks or dress. Overall, the interviews revealed that microaggressions are a far more prevalent form of gender harassment.

\subsubsection{Microaggressions}

Given the prevalence of microaggressions in scientific organizations $[19,20]$ and the numerous examples in the interview data, several survey questions focused on gendered experiences with microaggressions. Many of the sections in the survey had questions addressing types of microaggressions including S3: Gendered Experiences, and S6: Gendered Outcomes. Both of these sections concluded with an open-ended response question that elicited many specific examples microaggressions.

- S3Q12: Do you have any additional comments about your experiences at NIST based on gender that you would like to include?

- S6Q4: Do you have any additional comments about the Work Environment at NIST that you would like to include?

While the term microaggressions did not appear in the survey, staff at NIST are aware of microaggressions. In fact, several survey participants both women and men used the term in their comments and cited examples.

I will say that by now most (male) scientists at NIST know better than to say outright sexist things in public or to do outright illegal things like sexual assault. But daily microaggressions against female scientists are pervasive. Condescending behavior towards women is common. Credit for work/ideas is often stolen from female scientists by male scientists, or automatically attributed to the male scientists on the project. I know that there are (male) scientists who do these things to other men as well, but my observations over the years has been that these people are selective in their targets, and if you are the lone woman it is almost certain that you will be a target. I know other women at NIST who experience these things all the time too. (S6Q4:P2271:F)

The culture is toxic and just bad at NIST. The amount of microaggressions I have witnessed and experience is gross and horrible. (S2Q9:P2171:F)

It is not usually an issue in terms of overt sexism, but there is definitely many microaggressions and systemic problems hindering people of color and women's potential at NIST. (S6Q4:P2184:M)

While many recognized that the questions were addressing microaggressions, the differences in response patterns between the genders to the questions on microaggressions is striking. The following sections explore the differences in the response pattern between the genders.

\subsubsection{Subtle Putdowns}

In addition to the two questions specifically on harassment, several questions focused on behaviors associated with types of exclusion including being interrupted and not allowed to 
finish your thought, or not invited to a meeting or included on an email. All are forms of subtle putdowns.

- S3Q1: I believe I have been interrupted in a meeting because of my gender.

- S3Q2: I believe I have been excluded from meetings/emails or other work-related activities because of my gender.

For these questions, participants were provided with three response options:

(1) No, has not happened, (2) Yes, has happened at least once, (3) Yes, has happened more than once.

Both of these questions were found to be statistically significant (Figure 19). While no more than $5 \%$ of men responded affirmatively to these questions, nearly $30 \%$ of women reported having each of these experiences. About half of the women reporting being interrupted in meetings compared to only $5.0 \%$ of the men $\left(\chi^{2}(1)=288, p_{\text {corr }}<0.001\right)$. About $22 \%$ more women have experienced being excluded from meetings/emails or work-related activities than their male counterparts $\left(\chi^{2}(1)=106, p_{\text {corr }}<0.001\right)$.
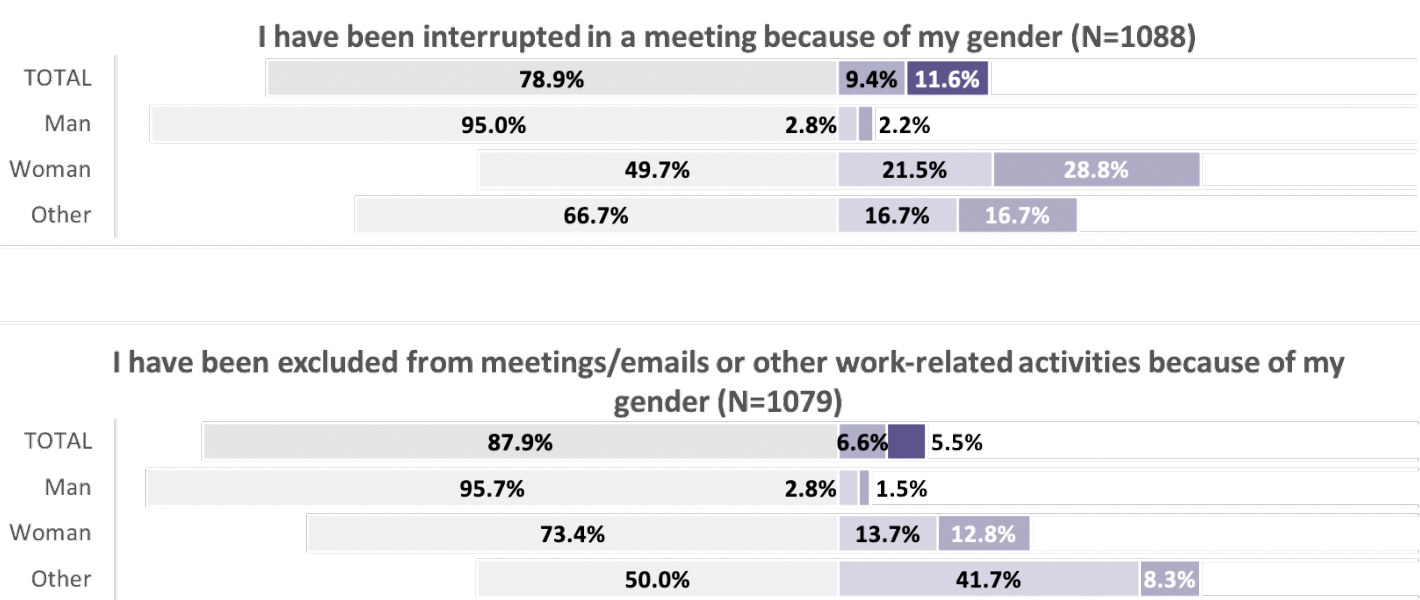

Figure 19 : Subtle Putdowns

The graphs show the distribution by gender where responses range from No, has not happened (left most /gray), Yes, has happened at least once, to Yes, has happened more than once (right most/dark purple). The number of respondents is shown in parentheses.

The interview data and the survey data in response to S3Q12 (Do you have any additional comments about your experiences at NIST based on gender that you would like to include?) provide ample evidence that subtle putdowns are common at NIST. Men as well as women commented on observing these behaviors in meetings.

It feels like an old lady's tale, but you'd be asked for your input, and you would say it, and the person sitting next to you, who in most cases is a man and in my case is basically 
always a man, would say the exact same idea, and someone would complement the man for that. That never felt very good. (INT:P105:F-42:00)

In every meeting I have attended over the years, women are usually pushed aside as the alpha males work to dominate the meeting. The women (and men) who have experience with this learn how to engage in these meetings. As a non-alpha male, I've been very frustrated over the years at my own experiences, but more so watching the women get ignored. This is a fact of life that I've seen everywhere. Some places are better than others but it's always there. (S3Q12:P1201:M)

Unfortunately, both women and men believe that the subtle nature of these behaviors make it virtually impossible to address. In fact P2640:F below describes that even trying to point out subtle harassment is met with questioning, additional putdowns, and general disrespect.

While those at NIST are too savvy to discuss inappropriate touching, comments about the way you dress, comments about body parts, etc., they regularly engage in discrimination in more subtle ways. Bringing this up seems impossible to do in this atmosphere.

(S3Q12:P2671:M)

A lot of the harassment is subtle and if you point it out the mostly male environment acts like you're crazy. (S3Q12:P2640:F)

Despite the subtle nature of this type of harassment the impact of these disrespectful behaviors is considerable. Dr. Paula Johnson, President of Wellesley College and Co-Chair of the NAS Report on sexual harassment of women [19], stated in the documentary, Picture a Scientist, that "consistent gender harassment actually has the same impact as a single episode of unwanted sexual attention or coercion" [20 timestamp 22:33].

\subsubsection{Questioning Competence}

In addition to the subtle putdowns, the interview data revealed interactions where women were treated with a more blatant lack of respect by their male counterparts. Common examples of this form of microaggression include questioning women's competence and assigning "housekeeping" chores to female staff.

The survey questions explored these behaviors by examining whether NIST staff believe their judgement has been questioned based on gender (S3Q4). This question was followed up with question S3Q6: I have been treated differently based on my gender, and S3Q7: I have witnessed men and women being treated differently. For these questions, participants were provided with three response options: No, has not happened, Yes, has happened at least once, Yes, has happened more than once. The differences in the answers to all three of these questions were found to be statistically significant.

Approximately $50 \%$ of women have had their competence questioned compared to only $3.3 \%$ of men $\left(\chi^{2}(1)=286, p_{\text {corr }}<0.001\right)$, Figure 20 . 


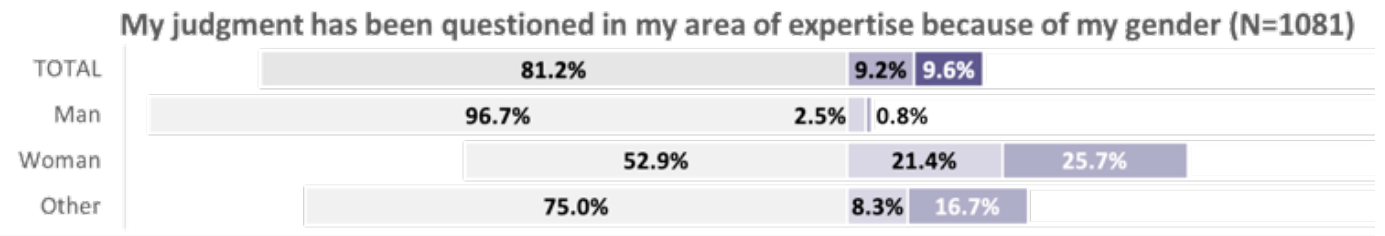

Figure 20: Questioning Competence

The graph shows the distribution by gender where responses range from No, has not happened (left most /gray), Yes, has happened at least once, to Yes, has happened more than once (right most/dark purple. The number of respondents is shown in parentheses.

Beyond subtle putdowns, the interview data revealed many interactions where women were treated with a more blatant lack of respect by their male counterparts. A common example in both the survey and interviews is questioning women's competence.

My experiences include a period of time where a co-worker kept telling dumb-blonde jokes in front of me with other colleagues present. (Off-color jokes are somewhat common since the boundary between work-friends and professional colleague can be blurry, and so these things are viewed as alright.) The same co-worker told me that I used my pregnancy to sit in my office and refuse to do work. I have witnessed other more offensive comments directed to or about other female colleagues. The most commonplace occurrences involve out-shouting or questioning competency in public. (S3Q12:P1780:F)

And this guy was asking us a question. And I answered it. And he looked at the guy and said, "Is that true? Is that correct?" And the guy was like, "What? She just gave you the answer." "I know. But I want to make sure it was right," like I didn't know the answer. And he was only looking at the guy and I'm answering the question. (INT:P013:F$48: 28)$

The women describe a continual battle to prove their competence irrespective of their positions. But establishing credibility is only the first step. Women also fear they cannot make mistakes. They believe they are continually being scrutinized and judged. In addition, to proving their competence women must also balance "the housekeeping chores" of the organization. These additional expectations further burden women.

Differences are subtle. When evaluating work systematically peers are fairly good about equity in analyzing accomplishments. However, there are a million unacknowledged judgments made that skew substantive opportunities towards men. As a single example, when an event needs to organized, or a scientist is required to take on a duty with administrative burden (eg DSR, Quality Manager, etc), the first person to be approached seems much more likely to be female. Why? These important roles need to be filled, and may even be recognized in promotion decisions, but they put a burden on the scientist 
that impacts their ability to pursue research opportunities (which are more widely recognized accomplishments at NIST). (S7Q14:P1280:M)

Women are frequently pushed into more service oriented tasks out of a sense of obligation and a need to show competence. These roles take away from our ability to do science and are not respected/rewarded in the same way that individual scientific exploits are. Even if management value these contributions, colleagues overlook the excess service work and develop an unfavorable opinion of female colleagues. To better promote equity and diversity, effort needs to be put toward developing women as scientists and promoting their work. (S7Q14:P2308:F)

Both men and women in the survey responses recognized the cost to women of the housekeeping and service oriented tasks. Much like P2308:F above, women once again are trying to "show competence" thus finding it difficult to decline or sidestep these tasks.

In addition, to questioning competence women are also treated differently based on their gender. Over $50 \%$ of women and those of other gender have experienced being treated differently because of their gender compared to only $20.5 \%$ of men $\left(\chi^{2}(1)=133, p_{\text {corr }}<\right.$ 0.001 ), Figure 21.
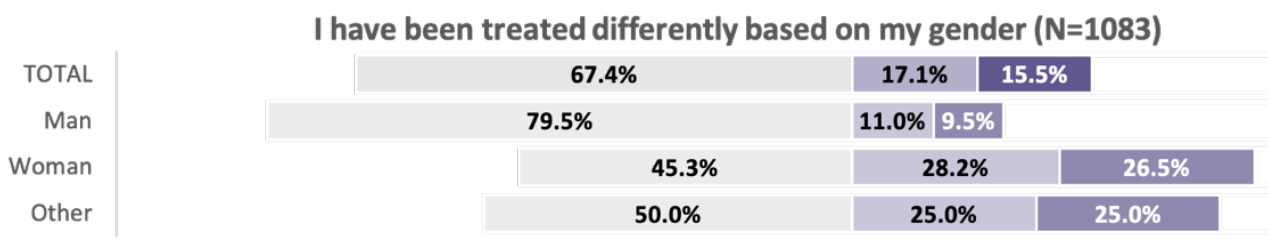

Figure 21: Treated Differently Based on Gender

The graph shows the distribution by gender where responses range from No, has not happened (left most /gray), Yes, has happened at least once, to Yes, has happened more than once (right most/dark purple). The number of respondents is shown in parentheses.

Furthermore, the majority of respondents have witnessed women and men being treated differently. There is a significant difference in the percentage of men $(51.0 \%)$ and women $(64.2 \%)$ reporting witnessing such situations $\left(\chi^{2}(1)=18, p_{c o r r}<0.001\right)$, Figure 22 . 


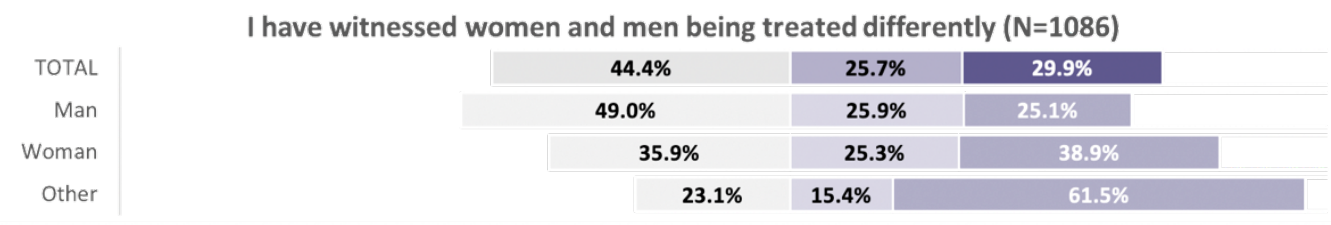

Figure 22: Witnessed Different Treatment Based on Gender

The graph shows the distribution by gender where responses range from No, has not happened (left most /gray), Yes, has happened at least once, to Yes, has happened more than once (right most/dark purple). The number of respondents is shown in parentheses.

There were many examples of a blatant lack of respect for women in the open-ended responses to S3Q12: Do you have any additional comments about your experience at NIST based on gender that you would like to include? In fact, both men and women described instances where women were "verbally beaten down" or where women were "treated as inferior and stupid."

I have seen women verbally beaten down by group leaders and former division chiefs because of their gender. They were accused of being too soft, or compassionate, or too interested in the well-being of the employee. One division chief in particular decided to put himself in the lead role at an international conference rather than his group leader who is an internationally recognized expert, but a woman. That's the only way I can explain it. This was a travesty and I lost a good deal of respect for NIST as an institution when it happened. These days, there is a half-hearted effort to make the pendulum swing the other way. (S3Q12:P1447:M)

I have had two different individuals in my division over the years that were fine with male employees and treated female employees like dirt. They did not touch or make comments about the women's bodies, but they constantly treated them as inferior and stupid no matter what. They were insulting and demeaning. One younger female guest researcher ended up with an ulcer from the treatment of one of these men. In both cases, NIST management simply changed the situation to where these men no longer had female subordinates and kept going. It was good to remove the female subordinates from the situations, but there were no further repercussions for the men. There were no HR actions, no nothing. They were allowed to keep their jobs and were not punished in any other way. (S3Q12:P1596:F)

The collective data paint a picture of women at NIST who continue to struggle for acceptance and are measured against tougher standards than their male colleagues. 


\subsubsection{Mommy Track}

In the interviews women expressed all sorts of concerns about having children and working at NIST, from the lack of role models to the challenge of balancing career and family to the fear that motherhood would derail their opportunities for advancement. A review of United States (US) longitudinal survey data found that approximately $43 \%$ of new mothers and $23 \%$ of new fathers leave full-time STEM employment after having children [21]. The proportion of mothers who leave is nearly double that of fathers. Motherhood is seen as a driver of gender imbalance in STEM employment.

Overwhelming, both women and men (nearly $91 \%$ ) agree that their need to balance work and other life obligations is supported at NIST. Only about $25 \%$ of men and women believe that using the available workplace flexibilities can negatively impact one's career. But about $40 \%$ of women and men believe working long hours at NIST is an important sign of commitment. The open response comments illustrate the struggle of demonstrating commitment while using the available workplace flexibilities.

Discrimination based on the impacts on career of providing childcare (which disproportionately falls on women in our society) are a definite issue. On question 10, "Working long hours is an important sign of commitment," I disagree but feel that most managers believe that (and overall NIST is insufficiently staffed, so people have to work more than we are paid to achieve the mission, regardless of gender). (S7Q14:P2595:M)

Working alternative schedules and teleworking to support my family has been a detriment to my career path. On years I took maternity leave, my performance was impacted although I completed all assignments throughout the performance year. I was told I spent too much time away, frequently pressured to return during maternity leave of only 8-12 weeks (depending on the child). Having a family, and needing flexibility, has affected my performance consistently. (S7Q14:P1181:F)

I have a school-aged child. And while I have encouraged those who work for me to take care of the paid dependent care leave during this pandemic, I have not done so myself because I am concerned it will make me seem less committed than my colleagues who don't have young children. I also believe NIST puts a high premium on those who work more than 40 hours a week. (S7Q14:P1328:F)

Balancing parenthood and work is seen by many as a detriment to career advancement at NIST. Nearly $40 \%$ of women (and over $36 \%$ other gender) believe that women with young and school-aged children are considered to be less committed to their career than women without children, which is significantly more than men $\left(15.9 \% ; \chi^{2}(1)=71.9, p_{\text {corr }}<\right.$ 0.001 ), Figure 23. 


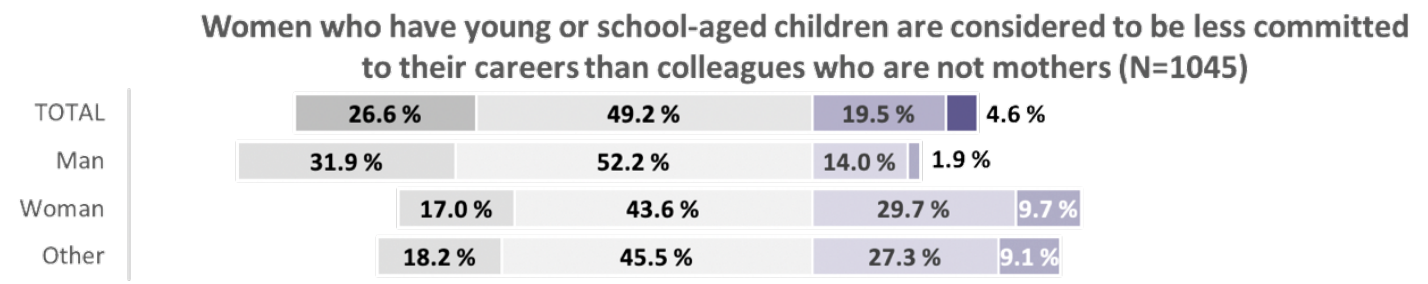

Figure 23: Women with Children and Commitment to Careers

Graph shows the distribution of responses by gender. The scale ranges from "Strongly disagree" (dark gray) to "Strongly Agree" (dark purple). The number of respondents is shown in parentheses.

\section{Women provided numerous examples across the open-ended survey questions of how motherhood impacted their careers.}

Most of the things that I have been overlooked for is because of motherhood. Having children slows down your career as a woman because of the time for sick leave/maternity leave, lactation, and childcare responsibilities. There is also less time to travel or to network outside of work hours because of childcare responsibilities. These are critical for establishing a presence in the field and in the workplace which puts you in the position to be considered for special projects. (S3Q12:P1544:F)

Young parents are treated differently at NIST. For men, coming into fatherhood is seen as a couple of weeks where they may be preoccupied but for women, its career ending. I was up for a promotion at NIST then I got pregnant. When I was 8 months pregnant, a senior manager stated in a meeting with me and my colleagues that I probably didn't want to go for a promotion because of my life changes. No one stood up for me, they just hung their heads and the conversation moved on. I was dumbfounded. It seems that managers are not trained in what constitutes discrimination against pregnant workers. The promotion opportunity was never posted so no discrimination actually took place. Additionally, services and information geared toward helping new parents is lacking at NIST. It's hard for me to believe that 10 years post the ACA requiring federal agencies to let employees have lactation space and time to pump that there are still employees denied the ability to express milk at work- yet I hear stories from female colleagues about this all the time. In addition to that, regular spaces are not set aside across the Gaithersburg campus that are private, convenient, and able to be scheduled for new moms to express milk- why is this so hard to do? (S7Q14:P1180:F)

You can stay in your current job at a pay band IV, working four days a week like this other person was doing, or you can revert to a pay band III with a three-day a week schedule, and we will move you back to operations." So, it took me about 12 seconds, and I said, "I will take the pay band III in operations." And my boss was shocked that I'd willingly take a downgrade and move into a different job because I wanted part- 
time. She did not understand this whole concept of different career goals.

(INT:P105:F-8:33)

While women with children are perceived as less committed and their careers are negatively impacted the same is not true for men. Given a similar question concerning men's commitment, the majority of respondents (about $87 \%$ ) of both men and women do not believe that men with young and school-aged children are less committed to their career. Only $9.1 \%$ of women and $13.7 \%$ of men agree that men with children are considered to be less committed (no statistical difference.)

The perception is real - men who are involved parents get kudos, women get labeled as unreliable/uncommitted/unprofessional. (S7Q14:P1930:F)

I have been at NIST for more than 30 years, so I'm hoping things have changed. I began my career with three successive term positions because I was 'a woman of child-bearing age,' making my group leader reluctant to offer me a permanent position.

(S7Q14:P1303:F)

While the last comment referred to the perception of women of child-bearing age 30 years ago the perception of being unreliable, uncommitted and unprofessional still has young women today at NIST questioning starting families for fear it will impact their career opportunities.

I am extremely concerned that if I choose to start a family I will not be given advancement opportunities at NIST. (S7Q14:P1499:F)

I'm in an interesting spot. The next phase in my life would be, I want to start a family. And I feel a ton of pressure in-- I don't know if it's just in STEM or in doing that. I can't say I've ever been to a big talk where an individual was pregnant visibly, where I could tell she was maybe far along....So I don't know how being out of here for, say, maternity leave will impact people's impression of me or my work performance at that time.

(INT:P005:F-49:04)

The experiences of women and motherhood at NIST are consistent with the STEM literature where motherhood is seen as a driver of gender imbalance.

The impact of the microaggressions is wide ranging. Not only do microaggressions and disrespectful behaviors harm women and other disenfranchised groups, but also harm the organization. According to [22], persistent disrespectful behavior impedes communication and collaboration, undercuts individual contributions, undermines staff morale, creates an unhealthy or hostile work environment, and causes some to abandon their profession. Disrespect causes the recipients in this case women at NIST to experience fear, anger, shame, confusion, uncertainty, isolation, self-doubt, depression, and a whole host of physical ailments such as insomnia, fatigue, nausea, and hypertension [22] . 


\subsection{Gendered Outcomes}

The culture at NIST contributes to men and women having different types of experiences, which often lead to gendered outcomes. Some of these have been discussed previously in this report, such as those related to hiring, retention, and promotion, or what happens due to experiences of harassment or exclusion, to name just a few. Other outcomes rely on gendered perceptions and assumptions produced and reinforced by the culture at NIST.

One very real outcome is whether or not women and men are respected, valued, and treated equally at NIST. Several questions in Section 7 of the survey ask participants to what extent they agree or disagree with these following statements.

- S7Q11: Women and men are respected equally.

- S7Q12: Women and men are valued equally.

- S7Q13 Women and men are treated equally.

It is striking that approximately $25 \%$ more men than women believe that both genders are respected equally $\left(81.5 \%\right.$ vs. $\left.56.5 \%, \chi^{2}(1)=76.3, p_{\text {corr }}<0.001\right)$, that they are valued equally $\left(80.2 \%\right.$ vs. $\left.53.8 \%, \chi^{2}(1)=81.9, p_{\text {corr }}<0.001\right)$, and that they are treated equally $\left(68.9 \%\right.$ vs. $\left.45.4 \%, \chi^{2}(1)=57.5, p_{\text {corr }}<0.001\right)$. These questions all show significant differences in the responses between men and women.

TOTAL

Man

Woman

Other

TOTAL

Man

Woman

Other

TOTAL

Man

Woman

Other

Women and men are respected equally ( $\mathrm{N}=1066)$

\begin{tabular}{|c|c|c|c|c|}
\hline \multicolumn{3}{|c|}{$\mathbf{4 . 6} \%$} & $22.7 \%$ & \multicolumn{2}{|c|}{$52.2 \%$} & $20.5 \%$ \\
\hline & $1.7 \%$ & $16.9 \%$ & $56.7 \%$ & $24.8 \%$ \\
\hline $7.7 \%$ & $30.8 \%$ & $30.8 \%$ & $30.8 \%$ & \\
\hline
\end{tabular}

Women and men are valued equally $(\mathrm{N}=1059)$

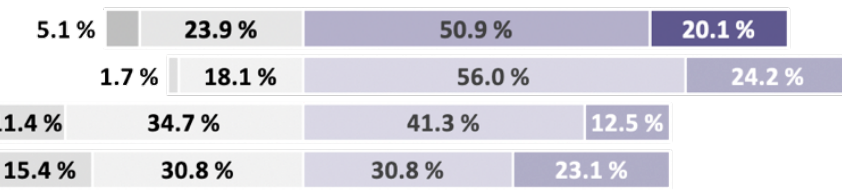

Women and men are treated equally ( $\mathrm{N}=1058)$

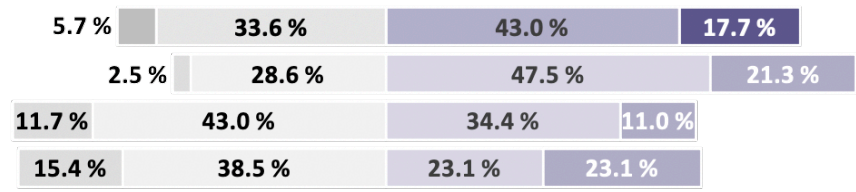

Figure 24: Comparison of Value, Respect and Treatment

Graphs show the distribution of responses by gender. The scale ranges from "Strongly disagree" (dark gray) to "Strongly Agree" (dark purple). The number of respondents for each question is shown in parentheses. 
These findings highlight an assumption of gender neutrality where gender is invisible, as are the effects it has within the organization. One interview participant called it a "huge blind spot."

...but I do think as an organization we ignore that it \{inequity\} exists. I do think that we have a huge blind spot for both, I'll say, the equity side of advancement and the impact. I think it's impossible to correct when all of our leadership is men, because I think it's just-it is things that they don't even think of because they just haven't been in the situation... (INT:P104:F-50:48)

I am on the fence about the questions above. During the past 30 years, I have seen changes at NIST on many of those issues but there is still room for improvement. Unconscious bias is still a major issue when it comes to treatment of and opportunities for women vs men in the workplace. (S7Q14:P1780:F)

This blind spot, or unconscious bias, allows men to believe that NIST is gender egalitarian, that gender does not influence experiences and outcomes. Kelan argues that "there is much evidence that people construct their workplaces as gender egalitarian" and that "these forms of discrimination are often invisible to people working" there [23, p. 198].

While the majority of men and women agree that both genders are respected, valued, and treated equally, it is important to consider that over $40 \%$ of women disagree with these statements. One must ask what happens in an institution when employees believe they are not respected, valued, or treated equally.

Qualitative data from interviews and open-ended text responses highlight how participants view the ways in which women and men are respected, valued, and treated differently. One survey participant put it quite succinctly.

There is no equality! (S7Q14:2643:F)

Other qualitative data provide greater insight into why employees, especially women, perceive that respect, value, and treatment are not equitable.

And I really wonder, as an organization, how can we claim that we really are equitable, that we really are inclusive if we are not holding bad behavior to account (INT:P105:F01:01:17)

Often NIST feels like it is still in the Mad Men era in the way that men and women (and minorities) are treated. (S7Q14:P1873:M)

My experience is that women and men are treated equally, but are not valued equally or respected equally. The opinion of a man is held higher than that of a woman.

(S7Q14:P1905:F)

When women and men are not respected, valued, and treated equally it creates the conditions that lead to unequal opportunities and outcomes. 


\subsubsection{Working Harder}

Another very real outcome of the culture at NIST is how much effort it takes for an employee to be successful. In S4:Q1, participants were asked to what extent they agreed or disagreed with the statement below.

I believe I have to work harder than my colleagues for my work to be recognized/accepted.

Overall, $29.1 \%$ of respondents agree or strongly agree with the statement. Significant differences were found between the responses of women and men to this question, with $49.1 \%$ of women agreeing that they have to work harder and only $6.9 \%$ of men agreeing with the statement $\left(\chi^{2}(1)=244, p_{c o r r}<0.001\right)$. Thus, women are significantly more likely to believe that because of their gender they have to work harder than their colleagues for their work to be recognized and accepted.

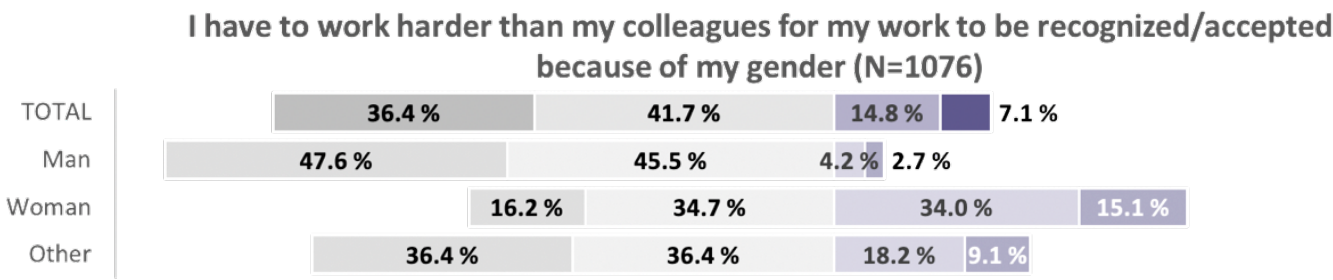

Figure 25: Women Work Harder for Recognition

Graph shows the distribution of responses by gender. The scale ranges from "Strongly disagree" (dark gray) to "Strongly Agree" (dark purple). The number of respondents is shown in parentheses.

Both interview data and open-ended survey responses indicate that women believe they often have to worker harder.

You have to work twice as hard; you have to be twice as good, and that sort of thing. And you're not as able to make mistakes because the focus gets put on you a little bit more quickly than a white male for example. So, part of that is something that's ingrained in our community. (INT:P008:F-49:39)

I worked for several years as the only female and had to go out of my way to accommodate the majority (men) in my division. It wasn't until 8 years later (when another female was hired) that things started changing. They started making accommodations for that female and the new management apologized that I had to put up with some of the inconveniences of being the only female for so long. At times it was harder to do my job because I had to go through extra precautions and steps to get the job done. (S3Q12:P1891:F) 
I have read that it is common for women in technical areas to have to work five times harder to get the same recognition as men. I have experienced this constantly over the $20+$ years I have been at NIST. I must justify my technical approach, my technical conclusions, am generally omitted from decision making, and my contributions have been omitted from technical publications. When I ask management for help in solving some of these issues, the response typically implies that I am being overly emotional or not rational. Rarely has an issue been addressed with structural change. I have experienced that when male colleagues express issues, their concerns are addressed and changes are made. (S7Q14:P2669:F)

As the participants above note, women often have to work much harder than men to be recognized. They also have to work hard and take extra precautions to not make mistakes, since these could have dire consequences. As P2669 notes, they must justify their work, are often not part of decision making, and their contributions are not seen or valued. If they question these experiences, they are painted as emotional — as feminine — rather than as the desired rational, masculine, scientific actor.

\subsubsection{Missing Out on Opportunities}

In addition to having to work harder, another very real outcome is missing out on opportunities that could further one's career. P2699 above notes how she has been omitted from decision making and how her contributions have been omitted from publications-both of which could contribute to her missing out on future work opportunities. In question S3Q5, participants were asked whether they believe they had missed out on work opportunities (for example, chairing committees, leading projects, or working on key assignments) because of their gender. Response options were: 1) Yes has happened more than once; 2) Yes has happened at least once; or 3) No has not happened. Significant differences were found between women's and men's responses for this question $\left(\left(\chi^{2}(1)=100, p_{c o r r}<0.001\right)\right.$.

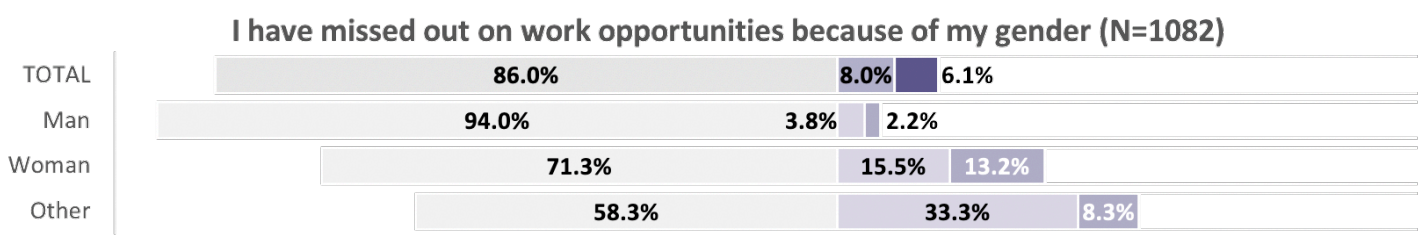

Figure 26: Work Opportunities

The graph shows the distribution by gender where responses range from No, has not happened (left most /gray), Yes, has happened at least once, to Yes, has happened more than once (right most/dark purple). The number of respondents is shown in parentheses.

Qualitative interview and open-ended survey data also support the idea that work opportunities are missed because of one's gender. 
Equitably, I can talk about developmental assignments. I can't say I've ever had a supervisor, with one exception, actually talk to me about developmental assignments and whether or not we should pursue them. So for example, I've never been through any of our leadership development programs. (INT:P105:F-36:01)

I believe that my OU seeks out women to promote, but I think women have less opportunities to do lab work, serve on international committees, and generally do the work that increases their qualifications for management. If you are constantly asked to organize parties, you have less time to do real work. If have less opportunities to do lab work, you have less publications, you are less likely to be seen as a candidate for international committees. Those are areas where I see women being constantly left behind their male colleagues in my OU. (S6Q4:P2453:F)

As these participants note, there are many ways that women miss out on work opportunities. Women often bear the burden of taking on roles and duties that do not allow them to develop the skills that position them to take advantage of future opportunities. P1947 below highlights how the pay and promotion gap evident early in men's and women's careers serves to limit opportunities for women at higher levels of leadership.

There is a consistent and pervasive gap in salary and promotion between men and women at NIST as a function of time and experience. Even if promotion at the top levels is fair and transparent, there is a reduced number of women positioned for those opportunities due to that gap. (S6Q4:P1947:F)

The human resources data certainly support this, showing very few women at the highest levels of leadership at NIST [1].

In addition, as P2699 noted in a previous comment, when she questions missing out on opportunities, she is called emotional, which positions her as not deserving of the opportunity. Below, interview participant P101 and survey participant P1215 also highlight how women are perceived when they advocate for opportunities for themselves.

Women don't seem to get taken seriously. And if you do try and stand up for yourself, you get labeled as troublesome. Even if all you did was say "Hey, I think I would be really good at this, and this is kind of the research I've been doing, so why don't I fulfill that role?" That would be considered aggressive. (INT:P101:F-5:55)

Women are described as "bossy, aggressive, 'only looking out for number one' instead of being a 'team-player'" when seeking advancement opportunities or asking pointed questions about career growth. (S7Q14:P1215:F)

Identifying women as emotional or troublesome when they question missing out on opportunities is one way to justify limiting their advancement. 
While the overwhelming majority of the comments about missed opportunities came from women, a few male participants also noted they had experienced missed opportunities due to their gender.

Some years ago, I had my supervisor tell me directly that he was not advocating for me for an important opportunity because he felt there needed to be more women in visible roles in science. (S3Q12:P1419:M)

P1419 believes he missed out on an important opportunity because it was given to a woman. The implication here is that the opportunity was unfairly given to a woman in order to heighten the visibility of women in science at NIST. Employee perceptions about the fairness of how opportunities are allocated should always be addressed, as they have the opportunity to undermine diversity and inclusivity and in this case women's credibility.

\subsubsection{Getting Credit for One's Work}

Another outcome that can place employees at a disadvantage is when their work is discredited in some way. In S3Q3 participants were asked to what extent they believe others have taken credit for their work because of their gender. Significantly more women $(36.6 \%)$ than men $(2.8 \%)$ agreed or strongly agreed with this question $\left(\chi^{2}(1)=203, p_{c o r r}<\right.$ 0.001). The large gap in men's and women's responses speaks loudly about differences in experience and outcome.

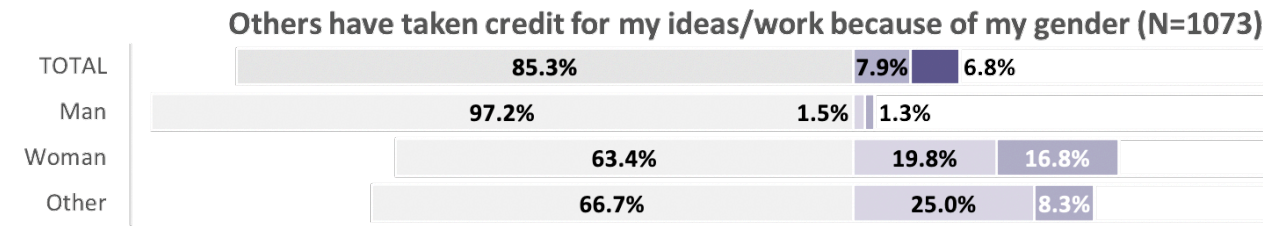

Figure 27: Others Take Credit for Ideas

The graph shows the distribution by gender where responses range from No, has not happened (left most /gray), Yes, has happened at least once, to Yes, has happened more than once (right most/dark purple). The number of respondents is shown in parentheses.

Similar ideas surfaced in the qualitative data from both interviews and open-ended survey responses.

I have repeatedly had others (male coworkers) take credit for my work and ideas, and had credit for my work/ideas attributed to them by others (also men). I have also repeatedly had my ideas/work questioned or disparaged by (male) coworkers. So it frequently occurs that my work/ideas either gets questioned/disparaged or stolen. .... It has gotten to the point where I am actually SURPRISED if this does not happen. I have also often had (male) coworkers try (and in some cases succeed) in taking over projects that I started and which I did not involve them in. This experience of repeatedly having credit for my 
work/ideas stolen or automatically given to my male coworkers, or having male coworkers seek to take over control of projects which I started and succeed in doing that, makes it very difficult to want to collaborate. (S3Q12:P2271:F)

I'm tired of other people being promoted that don't pull their weight compared to hard workers like me. I am tired of being a ZS staff member and feeling left-out at times or feel like my opinion doesn't matter (i.e. I'm never asked for an opinion or I share something I know, yet management or people above my pay grade asks someone else and that "someone else" gives management/employees above my pay grade tells that person THE SAME THING what I said). (S2Q9:P1988:F)

When someone else takes credit for your ideas/work, you are likely to miss out on future opportunities since your own accomplishments have not been recognized or rewarded. You may also be less likely to collaborate and more likely to become frustrated with your colleagues and your work - neither of which contribute to a positive work situation.

\subsubsection{The Ultimate Outcome}

As noted above, some women reported that they had to work harder than their colleagues, that they had missed out on work opportunities, and/or that others had taken credit for their ideas.

Perhaps one of the most important questions is whether these differences in experience and outcome would influence employees to leave NIST. In S6:Q3, participants were asked about whether or not issues related to diversity had caused them to consider leaving NIST (Yes or No response options). If they answered Yes to this question, they were asked an open-ended question about why they had considered leaving.

- S6Q3: Have you ever considered leaving NIST for reasons related to diversity?

- S6Q3a: If you have considered leaving NIST, why?

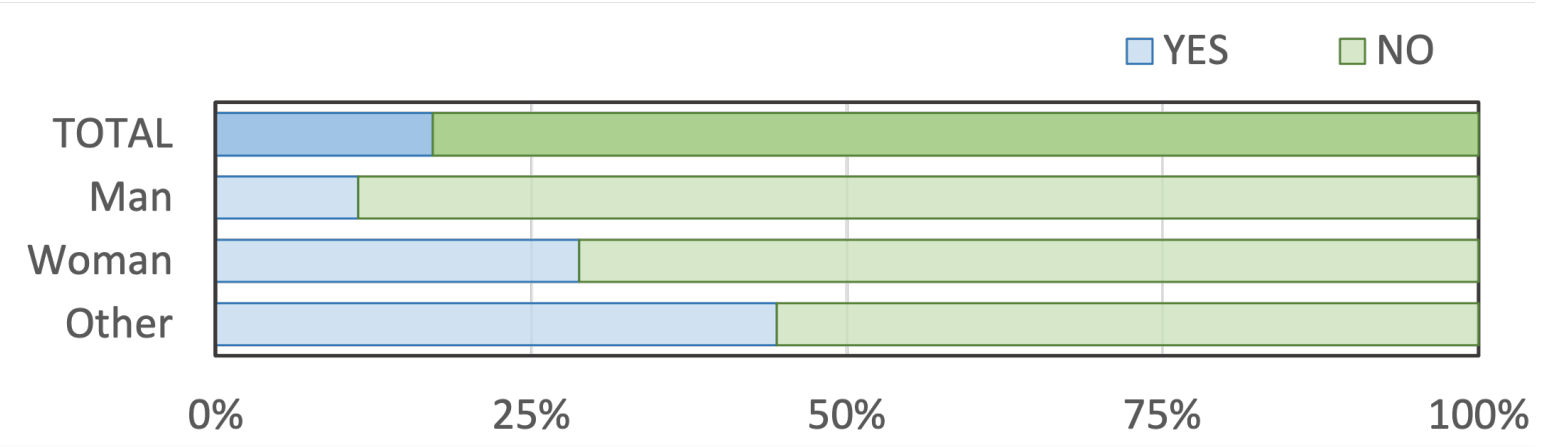

Figure 28: Leaving NIST for Diversity Reasons 
While only $11.3 \%$ of men report having considered leaving, the percentage is significantly higher for women at $28.8 \%\left(\chi^{2}(1)=28.9, p_{\text {corr }}<0.001\right)$. Given the experiences and outcomes women face, it is not surprising that they might consider leaving NIST in greater numbers than their male counterparts.

There were 92 open-ended responses to question S6Q3a about why participants had considered leaving NIST. This is almost $78 \%$ of the 118 participants who saw the question (who had responded Yes to S6:Q3). Responses ranged from comments about lack of opportunity, to lack of work-life balance, to discrimination and lack of respect. While comments came from both men and women, the majority of these comments were from women. Of the 92 responses, 61 were from women $(66.3 \%), 29$ were from men $(31.5 \%)$, and 2 were from Other $(2.2 \%)$.

NIST is a hostile work environment to Black people. Black people, and probably other minority groups, are "othered" in work and social settings. There is little-to-no diversity among leadership, and NIST does little to support its minority staff at any level of their career. Women in leadership are unicorns at NIST. I don't know the statistics, but I wouldn't be surprised if NIST leadership consisted of 70+\% white males.

(S6Q3a:P2020:F)

I was subjected to harassment at NIST that impacted both my professional life and personal life, it is hard not to consider leaving somewhere after experiencing that. (S6Q3a:P1815:F)

In my first year at NIST, my division chief made comments that I felt weren't appropriate and made me feel uncomfortable. It gave me pause, and generally made me think that -if this person felt comfortable saying those things around me -- they may not value my perspective or standing in the organization. This same division chief once asked me, in a one-on-one meeting, what I wanted to do "when I grew up." It made me want to walk out of the room, and I certainly thought about leaving NIST. (S6Q3a:P1926:F)

Comments mentioned in earlier response about workplace respect. Male colleague was consistently rude, demeaning, and horrible to work with for months. Supervisor didn't stand up for me, I had to put myself out there and stand up for myself. Risky business as a lower-on-the-totem-pole female. (S6Q3a:P1872:F)

I felt like leaving when I thought I was being passed up for an opportunity solely because of my gender. My first line supervisor was extremely supportive, however, and managed the situation very well. (S6Q3a:P2256:F)

Women considered leaving because they did not feel supported, did not feel valued and/or respected, and believed their opportunities were limited.

While most of these comments about leaving for reasons related to diversity were from women, some men also recognized how the patriarchal culture at NIST could contribute to 
many employees wishing to leave because they do not want to work for an organization that does not value and respect all employees.

NIST is just not changing fast enough with the outside culture to be a relevant and useful place to work in the near future. NIST leaders appear reluctant and scared to 'rock the boat' too much and anger retirement-eligible employees to create a lasting organization that meets the expectations of 21 st century employees. If they don't adjust and give real voice to women and minorities instead of lip service, we will all be complicit to work for an organization that continues to marginalize people, regardless of what NIST leaders say or think they are doing to improve culture. (S6Q3a:P2163:M)

Several interviews as well as other open-ended survey questions also highlight situations where women considered leaving NIST for reasons related to gender.

But because of his management style, I noticed that if any woman challenged him or spoke up for themselves, they were on his hit list. And so there was a great divide between the have and the have nots. If you-- he would conduct meetings that way. And I was like, "This is not good."... So I was, at that time, thinking fight or flight. That's what I was thinking, fight or flight because with the way that I am, I'm not going to be insubordinate. But I'm not going to be this submissive whatever... I was in the fight or flight. And I was like, "Okay. Do I need to--" and I started looking elsewhere.

(INT:P102:F-38:22)

Because of some of the hurdles I described. That was around the time where I was thinking, "Okay. I need to get out of here." It was a little-- it was very frustrating.... So yeah. Yeah. I have considered leaving. (INT:P008:F-26:05-28:05)

When I was a NRC postdoc, my officemate whom I really didn't know, was given almost no resources to do her work whereas another term employee (male), was. Their career trajectories diverged dramatically. I saw it as 'starve her of resources' and she will leave. My officemate eventually left. (S7Q14:P1419:F)

When women, and other minorities, make the decision to leave, it often hurts them as well as the organization. The loss of diverse perspectives and ideas can contribute to a loss in creativity and productivity [24]. In addition, role models are lost for other women and minorities in the organization.

While the majority of these responses were from women, some men also indicated they had considered leaving for reasons related to diversity.

I have thought about leaving because of the high focus on diversity and gender. (S6Q4:1526:M)

These responses often implied that somehow men were losing out as a result of the focus on diversity and gender, an argument that relies on an assumption of reverse discrimination, which will be discussed in the next section. 


\subsection{Reverse Discrimination}

Discrimination is about the unequal treatment of individuals based on their belonging to or affiliation with a historically marginalized group (for example women, or Blacks/African Americans). Discrimination is based on a system of unequal power relationships that have been institutionalized over time. Thus, reverse discrimination is considered by many to be a "myth" because it ignores the power and privilege dynamic that exists between the groups involved and assumes that the discrimination happens on a level playing field [25].

Brod and Kaufman argue that: "gender is a system of power and not just a set of stereotypes of observable differences between women and men" [26, p.4 ]. Because men have and hold power in this system, whether they want it or not, they cannot be discriminated against in the same ways as women. Yes, they can have experiences where individuals treat them differently or where they are harassed. However, these individual acts of prejudice do not constitute a system of discrimination.

We need to stop identifying racist and sexist behavior as only that perpetrated by bad individuals with intent to harm, and we need to understand the multitude of ways that Whites have been advantaged by their race, and that men have been advantaged because of their gender. White men are particularly unreliable and unaware as to how they have been afforded the many privileges that culminate in their status and power. So, if we are ever to make progress in identifying and dismantling the structure of white supremacy that is the norm today, we must begin by turning to POC and women to teach the White men how institutional racism and sexism negatively impacts their everyday experiences both in the workplace and in their personal lives outside of work. (S1Q5:P2182:M)

As P2182 notes in the open-ended response above, racist and sexist behavior is about much more than the individual behavior of "bad individuals," it is about a system of status and power that benefits some - even if they do not want those benefits - and disadvantages others.

While some men at NIST recognize the ways in which men and masculine ways of being are privileged, others believe they have been discriminated against because of their gender. Some specifically reference reverse discrimination while others talk more generally about how white males in particular are treated badly.

While gender discrimination is not acceptable we should not only focus on the impacts on women. I have seen and subjected to different treatment by women in my division. Reverse discrimination should not be acceptable either. (S3Q12:P1906:M)

I have witnessed a sort of reverse bias, where a female is given more leeway, less negative interactions, and greater pay and credit due to being female as opposed to what would happen with a male in the same circumstance. (S7Q14:P1826:M)

More than a few men perceived NIST's current emphasis on diversity as "threatening" to white men who they believe are seen as "bad people." 
White males are automatically determined to be bad people, in the wrong, insensitive, and undesirable employees. (S3Q12:P1674:M)

As a white male, I see efforts at "diversity" and "inclusivity" as threatening to me, in the following ways: 1) Being told that I have to "confront white privilege and male privilege on an hourly basis" -- I worked hard to get where I am. I did not grow up in an affluent family, I am the only one of four siblings to go to college, it bothers me greatly to be told that I am the beneficiary of some fictitious set of privileges. 2) Every time I hear about increasing diversity, I hear that as "White males need not apply". It is simply unfair to give a preference to certain races or genders simply because of their classification. Everyone should be treated equally, and we should aim for a color- and gender-free society. (S1Q5:P1830:M)

Here the construct of reverse discrimination is used to argue that policies or practices designed to combat institutionalized racism or sexism are "unfair" and position white men in negative ways. Certainly, the nature of power in men's lives is contradictory: while men are said to have power and privilege, not all of them feel powerful or privileged. These feelings must be acknowledged and addressed before any real change can occur.

Some male survey participants note that they believe women are favored to meet quotas, thus privileging women more than men.

I believe women have a greater opportunity to move into leadership positions when considering level of experience since NIST is trying to improve their numbers (S6Q4:P1259:M)

I believe women are offered slightly greater opportunity at NIST to move into positions of leadership in order to fill the statistically significant but superficial void, but that they have chosen not to for personal reasons. So this question is hard to answer on balance since "opportunity" here does not distinguish between what NIST management offers and what real life circumstances enable. (S6Q4:P2662:M)

Women are sometimes treated differently because of their gender per policies to increase the statistical representation of women in STEM and at NIST. (S3Q12:P2445:M)

These subtle references to affirmative action position women as the beneficiaries of an unequal system. In reality, affirmative action is about creating fair access to opportunities for all employees, in order to create a workforce that accurately reflects the demographics of the qualified available workforce. Within such a system, employees (or candidates) are not given opportunities they do not deserve or for which they are not qualified. Assumptions about quotas and numbers damage the ability of an organization to address discrimination and in this case call into question women's abilities and success.

While some of the comments, like those above, speak generally of women being favored, others focus specifically on how some men believe they have been specifically affected by reverse discrimination. 
Overlooked for assignments because I was not femail or a racial minority at NIST. (S6:Q3a:P1911:M)

Yes, I have personally been told to include a woman on a Committee_only_because of her gender. This is a classic example of gender bias, which is just morally wrong. (S3Q12:P1380:M)

These responses demonstrate ways in which individual men believe they have been wronged due to a focus on women.

While this section focuses on how reverse discrimination is used as an argument against addressing issues that women currently face at NIST, other responses from men indicate that they believe women should have access to more opportunities given their current underrepresentation.

During my day-to-day work, a large majority of the scientific staff that I interact with are men. I am not aware of what the NIST-wide statistic is for the representation of women and minorities in scientific positions, but based on my daily interactions, I would like to see more opportunities and positions given to women and minorities. I will say that it is good that my group leader is a woman, but I would like to see more diversity. (S2Q12:P1562:M)

I answered question 7 as no because the implication of the question is that men are treated better than women. From what I've seen, management is desperate to promote and hire women and minorities and gives them preferential treatment. I'm actually okay with that in order to balance out our gender/race statistics, but we do bias towards women. Were I a young white man I'd feel very disadvantaged in the current NIST culture. (S3Q12:P1542:M)

While individual men may support engaging in practices and policies that will address the underrepresentation of women at NIST, there is a need for systemic change to address the cultural components that create and perpetuate this underrepresentation. To be effective, this cultural shift must also address the varied reactions of men who may see these shifts as challenging their place in the status quo.

\subsubsection{Women and Reverse Discrimination}

Some women also articulate the idea of reverse discrimination in the open-ended survey data, sometimes arguing that "women are too sensitive" or that focusing on women and other minorities in hiring and promotion might overlook "non-diverse" candidates (which one can only assume refers to white men.)

Sometimes I feel like inclusivity and diversity is promoted so much at NIST that we might be overlooking an outstanding non-diverse candidate when filling positions at NIST. I believe in equal rights for all, not singling out any specific group. (S1Q5:P2333:F) 
I had some difficulty with these questions. I often think that women are too sensitive (FYI - I'm a woman!) and that they tend to assume negatively about comments made by others. I tend to think that people innocently say something until otherwise shown different. For example, I was told by one supervisor just after coming to NIST that I should have been a baker. For a long time, I steamed about it thinking that he meant that I would have made a better baker than an engineer; however, I came to know him better and now realize that he truly meant it as a compliment. He liked my baking. He didn't mean to imply that I couldn't be a good engineer. I'm also not sure if I missed opportunities due to my gender or if it was because the men surrounding me were much more assertive than I am. There were other times that I am sure that my supervisor (different one than above) purposely refused any promotion due to the fact that I had the nerve to speak up where at the same time, he respected that same quality in my male colleagues. At NIST, I've seen assertive women be labeled as "aggressive", "mean", "rude" where the same behavior in men would be labeled "assertive" "being direct", etc. (S3Q12:P1583:F)

For women to move forward they need to buy into the status quo as well. They don't want to be seen as having their positions due to their minority status versus their merit. Jorgensen [27] found that female engineers reject the idea that gender has had an impact on their career and do not want to be seen as a minority group in need of special attention.

\subsection{Bifurcated NIST: The Boys' Club and the Chilly Climate}

As previously discussed, gender is more than just the differences seen between women and men and the stereotypes often based on these. It is a system of power based on a gendered hierarchy that serves to advantage some while disadvantaging others. As detailed in the previous sections, this system of power has created a culture at NIST where barriers and facilitators for men and women at NIST operate very differently. Few barriers exist for men related to gender, while many invisible advantages act as facilitators. The opposite is true for women. Ultimately, the culture at NIST results in bifurcated experiences and outcomes for men and women, with a "Boys' Club" that supports men and a "Chilly Climate" that creates barriers for women. The Boys' Club privileges men and masculine ways of being, while promoting the outward appearance of neutrality. At the same time a Chilly Climate exists for women that often results in negative experiences including exclusion that serve to disadvantage women as a whole.

To understand a system of power, it is imperative to pay close attention to its beneficiaries. Several participants in both the interviews and the survey specifically identify and call out this system of power, noting how a Boys' Club exists at NIST. Some of these responses merely named it as a "club" or "network," while others provided more details on how the Boys' Club protects men and provides advantages for them, while at the same time disadvantaging and constructing barriers for women.

It's an old white man's club. Heterosexual males more specifically. (S2Q9:P2243:M) 
NIST is the standard bearer of "the good ole boy network" whereby those that "kiss the ring" are rewarded for their loyalty. I've seen lots of people who have "failed up" that continue to be on the payroll. Also, scientists are absolutely a huge piece of why NIST is so amazing - but they're not the only ones who work at NIST. The organization would do well to remember that. (S2Q9:P1663:M)

Working in a team environment gives rise to group think and sometimes a "boy's club". Though I have not experienced any gender-based exclusion from official business, I am not part of the so-called club and can therefore miss opportunities that may organically come about from more casual conversations. (S3Q12:P2054:F)

Some of the supervisors, especially in the finance area, are all products of the good old boys' club that was really rampant when I first came to NIST. And they believed that once you were on their team and they were your supervisor, that they owned you. And woe be it to you to want to move out, move up, move forward. (INT:P001:F-2:02)

As the interview and survey participants above note, the Boys' Club at NIST offers opportunities for men, especially for those who work to be in the "club." This club is premised upon the hierarchy of gender relationships that exist at NIST. Certainly, not all men benefit in the same ways from the Boys' Club, as evidenced by much of the data in this study. However, what is most important perhaps is the existence of the hierarchy and its effects, specifically on women.

As detailed throughout this report, the gendered hierarchy at NIST often provides a Chilly Climate that actively discriminates against women. This Chilly Climate is based on "patterns of inequitable treatment that, as they accumulate, inhibit women's confidence, self-esteem, and accomplishment'" $6, p .7]$ patterns that are sometimes overt and sometimes subtle. Just as it is important to examine those who benefit from the gender hierarchy, it is important to look at those who do not. Much of this report has shown the multiple ways in which this gender hierarchy exists and how it contributes to a Chilly Climate for women. It is not only women who recognize the Chilly Climate at NIST; some men clearly recognize their male privilege, and how it contributes to the Chilly Climate for women.

I am a White male. As such, I have been afforded enormous privileges throughout my life, including education and employment. Therefore, I strongly believe I have been treated differently based on my gender, as well as my race. I have not been treated poorly. In fact, it is quite the opposite. I have been treated very, very well. It has generally been to my advantage, but this does not make it right. (S3Q12:P2182:M)

In this open-ended survey response, $\mathrm{P} 2182$ recognizes his own privilege and how he has benefitted from it. Other responses identify aspects of the Chilly Climate women often experience.

In the last few years, I have heard of a startling number of experiences from my female colleagues at levels ranging from the benchtop up to interactions lab management. These experiences range from minor everyday (but valid) grievances to undeniable sexual 
harassment. These are not my experiences but it is important that everyone know that they are happening **regularly**. (S3Q12:P2346:M)

...the criteria for promotion within the ZP pay scale are based almost entirely on individual performance. I believe that this may negatively affect the advancement of women, since many studies have shown women to work in a more collaborative way than men. (S2Q9:P1489:M)

In their open-ended survey responses, these men identify ways in which the Chilly Climate negatively effects women at NIST. In addition, these responses begin to identify how men benefit, even if they don't want to, from their privilege. However, this type of awareness and recognition go a long way in dislodging the hierarchy.

Some of the things that act as facilitators for men function as barriers for women, such as assumptions of objectivity and meritocracy. Other barriers for women include things such as isolation and invisibility, lack of respect, and a focus on body/dress rather than intellect and work. These have all been discussed previously in this report, along with how they function to create barriers for women at NIST. However, in addition to creating barriers they contribute to an overarching Chilly Climate for women. Below, two interview participants describe in detail how women experience the Chilly Climate at NIST.

I probably, over the years, worked with three or four different women, total, out of a group of 50 or so people. And I can't recall a single woman I worked with ever being, like, "Oh, yeah. This is a great environment," or having them not have these types of issues at some point and feeling very-- like, everything you say gets interpreted in the stupidest way you could have possibly meant it. And it's really frustrating to try to feel like people actually take you seriously and treat you like a real researcher with potential. If anything, it seems like a lot of the women get treated like they are the secretary equivalent in a lab of doing the necessary but unimportant and unimpressive tasks. And so a lot of the women I know who do work at NIST, wind up getting pigeonholed into these projects that go nowhere, don't have a lot of publishing capabilities, and then NIST seems surprised that these women are frustrated and having trouble really promoting their careers further, when to an outside observer or somebody who actually is aware of the situation, it's pretty obvious why they're now having problems with it. And they're kind of expected to shut up and just take that role. And if you try to do anything different or expand or improve yourself, you get a lot of blow back. And people act like you're crazy, and you're too emotional to do this, and like, "Why is she upset, now?" (INT:P101:F$6: 59)$

I remember this plain as day. That's when he says, "You must learn how to toot your own horn young lady." That was his advice to me. And I said, "As you can see here, I have no problem tooting my own horn."... "But let me tell you what the real issue is. You're asking me to play a game that I don't even know what the rules are. And every time I think that I have reached the finish line, you keep moving the finish line. And as a matter of fact, let me make it a little bit more clear. It's like blowing bubbles, you go to catch it, 
and it disappears." He was silent. He got it. And my promotion went through.

(INT:P102:F-49:40 \& 50:00)

These participants lay out what the Chilly Climate looks like and feels like for women, including the consequences women face when they choose to question or disrupt the climate. Women are often required to accept the gendered hierarchy or else they get "a lot of blow back," even when they "don't even know what the rules are" for success.

Below, several open-ended survey responses also identify how women experience work life differently than men at NIST.

This feedback is not intended to imply that all women deserve to be promoted to senior staff/management levels; these promotions should be based on performance and qualifications. However, the barrier and expectations for women to be promoted to $\mathrm{ZP}-\mathrm{V}$ seem to be higher than their male counterparts. (S2Q9:P1215:F)

Although I believe that NIST views itself as an objective organization, it is quite subjective in my experience. There is a lack of transparency for most actions regarding career advancement and professional opportunities within the organization. The culture does not promote (or impart the skills to leadership to promote) interpersonal connection, which will help make the culture more inclusive and equitable. (S2Q9:P1479:F)

These participants identify some of the differences in the ways men and women experience work at NIST and some of the very real consequences of the Chilly Climate for women. Other responses show that even though participants may not experience the effects of the Chilly Climate themselves, they can recognize it.

I answer that women are treated differently on this page and throughout the survey because of what I see as the paucity of women in higher ranking positions at NIST (therefore something is wrong). I do not have direct experience with such things. (S7Q14:P2286:M)

The "paucity of women in higher ranking positions" leads P2182 to recognize that "something is wrong."

Unfortunately, NIST as an organization suffers from this gender hierarchy as well as the individual men and women who are not part of the "club."

NIST focus is on fundamental research. If you have a Phd, and are a white male, you rule. If you are female, non-white, veteran, or disabled, then you are considered a second class staff member. This is not surprising since this system was created and run by white male Phds. It is not going to change until the old white male Phds retire. NIST is missing out on the creativity and innovation that is born out of a diverse workforce. As long as white male Phds are charged with defining and evaluating the research, their bias will continue to be incorporated into the judgement. NIST is where it is at because of the team that drove it here. Expecting the same team to drive to a new destination against their values is rather silly. (S1Q5:P2618:F) 
Clearly there are different ways in which men and women experience work at NIST. When the organization privileges and rewards some ways of being over others, they do not have access to the full range of perspectives and ideas that a diverse range of employees bring to the organization. Addressing and dislodging the Boys' Club and eradicating the Chilly Climate will not only provide benefits to women, but also to the organization as a whole.

\subsection{Perceptions of NIST's Commitment to Diversity: The Ongoing Need for Change}

In the survey, participants were asked several questions about their perceptions of NIST's commitment to diversity and inclusivity. These questions asked about participant perceptions at the level of Senior Leadership as well as in their specific OUs. In addition to four quantitative questions, an open-ended text box provided unlimited space where participants could provide comments related to NIST's commitment. There were 347 responses to this open-ended question representing over $10 \%$ of the population of NIST federal employees, and $31.8 \%$ of the survey participants. Many of these responses were lengthy, over 200 words, with the longest response being 536 words and the average length being 58.78 words. These open-ended responses provide keen insight into participant perceptions about NIST efforts related to diversity and inclusivity.

- S1Q1: Senior Leadership is committed to inclusivity.

- S1Q2: Senior Leadership is committed to increasing diversity in the workplace.

- S1Q3: My OU is doing enough to increase diversity in the workplace.

- S1Q4: My OU is doing enough to promote inclusivity.

- S1Q5: Do you have any additional comments about NIST efforts related to inclusivity and diversity to include?

While the majority of the respondents agreed or strongly agreed with each of the four quantitative statements, there is a statistically significant difference between men and women's responses to all four, with significantly more men agreeing with each statement $\left(\chi^{2}(1)=22.1, p_{\text {corr }}<0.001 ; \chi^{2}(1)=18.5, p_{\text {corr }}<0.001 ; \chi^{2}(1)=15.2, p_{\text {corr }}<\right.$ $\left.0.001 ; \chi^{2}(1)=21.2, p_{\text {corr }}<0.001\right)$ ) for the four questions. Overall, these results show that men have a more positive perception about senior leadership and their OU's commitment to diversity and inclusivity than women do at NIST. Women are more likely to disagree that NIST is doing enough, at both the Senior Leadership and the OU level. This may be because they are more acutely aware of the effects gender has within the organization based on their experiences.

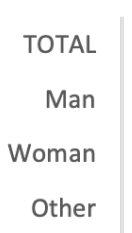

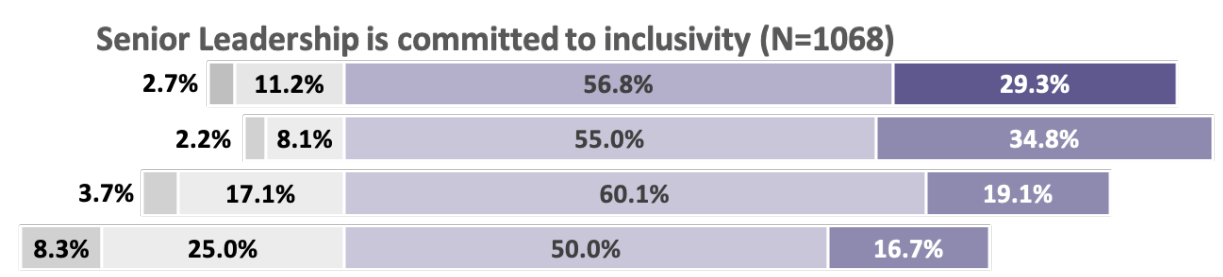

Figure 29: Commitment to Inclusivity 


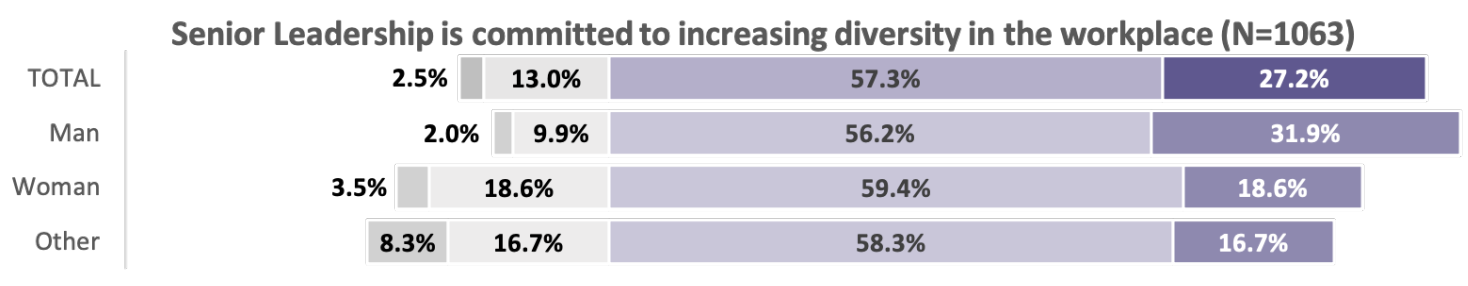

Figure 30: Commitment to Increasing Diversity

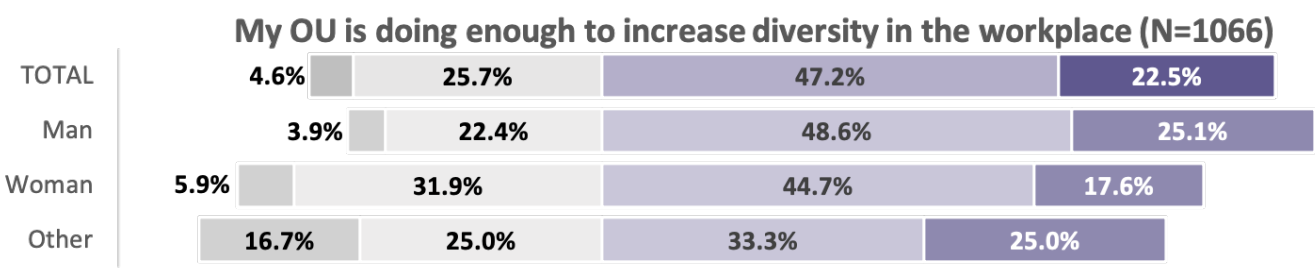

Figure 31: OU Commitment to Increasing Diversity

$$
\begin{array}{r}
\text { TOTAL } \\
\text { Man } \\
\text { Woman } \\
\text { Other }
\end{array}
$$

\begin{tabular}{|c|c|c|c|c|}
\hline \multicolumn{5}{|c|}{ My OU is doing enough to promote inclusivity ( $\mathrm{N}=1067)$} \\
\hline & & $22.8 \%$ & $49.2 \%$ & $22.7 \%$ \\
\hline & $4.5 \%$ & $19.0 \%$ & $50.6 \%$ & $25.9 \%$ \\
\hline $6.7 \%$ & & $30.0 \%$ & $46.5 \%$ & $16.7 \%$ \\
\hline & & $25.0 \%$ & $33.3 \%$ & \\
\hline
\end{tabular}

Figure 32: OU Promoting Inclusivity

Graphs show the distribution of responses by gender. The scale ranges from "Strongly disagree" (dark gray) to "Strongly Agree" (dark purple). The number of respondents for each question is shown in parentheses.

In addition, there is a significant difference in the responses to the two questions about increasing diversity. Significantly more participants agreed or strongly agreed that Senior Leadership is committed to increasing diversity $(84.2 \%)$ than agreed or strongly agreed that their OU is doing enough to increase diversity $\left(\chi^{2}(1)=65.0, p_{\text {corr }}<0.001\right)$. Regardless of gender, respondents are not as convinced that their $\mathrm{OU}$ is doing enough to promote diversity in the workplace.

While there were significant differences between the responses of men and women to these questions, the majority of respondents agreed that NIST Senior Leadership is committed to and that their $\mathrm{OU}$ is doing enough related to increasing diversity and promoting inclusivity. However, the open-ended survey responses paint a very different picture. This may be because participants believe leadership is trying to do something, but it is not enough to offset past imbalances and/or address the issue at the systemic level. 
I think during last year and this year my OU is doing a good job of addressing diversity and inclusivity. But I have been in this OU for over a decade and this is the first time I have seen this. So I hesitate to give high ratings until I see evidence that this interest is going to be sustained long enough to make real differences that affect the day-to-day working lives of women in the OU. (S1Q5:P2271:F)

While the OU may be doing enough to increase diversity and promote inclusivity, they could think outside the box to achieve even more. (S1Q5:P2251:F)

I have not seen much action to increase diversity in the workplace. They have talked about it loads, however there aren't new people of diverse backgrounds being hired right now. Both senior leaders and my OU does a good job of participating with various speakers/monthly focuses (ie Black History Month), however I haven't seen that creed reflected in the hiring of individuals and for that reason I am not choosing "Strong Agree" for either diversity question (2 \& 3). (S1Q5:P2204:M)

Moreover, participants may agree that leadership is committed, but they also question the extent and/or effectiveness of that commitment in the responses to the open-ended data. Many of the open-ended responses reflected on the superficial nature of NIST's commitment to diversity and inclusion, calling it a "joke," "performative," and "lip service," again calling the commitment into question.

This is a joke. Whenever there is a social flare-up, NIST decides it better do something, too. There is no diversity at NIST. Lots of talk, but little action. There is more attention paid to using politically correct language and watching out for "micro-aggressions" than there is with recruiting a diverse work force. Why are we taking a survey? We know there's a problem. I know exactly three black scientists and they've been the same three for at least a decade, two of them for two decades. I haven't seen an African American post-doc in my group or my division that I can recall. (S1Q5:P1447:M)

It's performative. There isn't enough driving inclusivity at NIST for it to be anything other than a check-the-box exercise. Look at the top leadership in the labs and OUs. They have no true motivation to promote inclusivity, beyond personal conviction (which, while nice, isn't a strong, consistent organizational driver). (S1Q5:1926:F)

I am concerned that while Senior Leadership pays lip service to these issues that they are not actually going to invest the needed energy and resources to drive the necessary cultural change. (S1Q5:P1888:M)

These responses identify NIST's approach to diversity and inclusivity as reactive versus proactive and question its authenticity. Other responses specifically question NIST's commitment and its desire as well as its ability to actually do anything related to increasing diversity and promoting inclusivity.

There is plenty of rhetoric but I question the commitment. I fear it will again be nothing more than halfheartedly implementing systems for show and not to solve real issues. If 
past is prelude, I full expect a whole raft of new symbolic and ineffective policies that will displease everyone, including me, but will show mostly symbolic results. The quantitative measures will improve but the new policies will not be color blind and will emphasize "positive" actions to be taken based on race and gender identification rather than eliminating "negative" actions based on race and gender identification. The beneficiaries of these new policies will be left wondering if they succeed on their merits and the others will be left wondering if they were denied equality based on their perceived benefits to these new gender and race equality "positive" policies. For what it is worth, I hope I am wrong. (S1Q5:P2066:M)

Whatever commitment leadership has to diversity, the demonstration of that commitment to diversity is sorely lacking. Just walk down the hallway at NIST and the majority of people you will see are white males. (S1Q5:P2180:M)

Again, there is a view that leadership says it is committed, but that it is often for "show." Other comments note how leadership's commitment may be a lot of "talk," but there is very little "action."

"Committed to" is very different from "doing anything." (S1Q5:P1284:F)

The "talk" is talked, but the "walk" is not walked. (S1Q5:P2263:F)

It's one thing for NIST leadership (the Director and three Associate Directors) to stand on a stage and tell us that they want inclusivity and diversity, but that is the extent of their participation. You never see them participate in any of the meetings or activities. I understand that they are busy, but it projects a view of division and disinterest.

Additionally, I have seen minimal action from all the meetings and discussions. It makes the effort seem very hollow. Why is NIST bothering to ask if they aren't going to do anything with our comments/suggestions or answer our concerns/questions. MML is the only place where I have seen proactive work towards understanding the situation so they can make efforts to fix the inadequacies. What is your 6 month, 1 year, 5 year plan??? SHOW us SOMETHING. (S1Q5:P2130:F)

Overall, this question elicited responses that questioned NIST leadership's commitment to diversity and inclusivity. Responses also clearly indicate that many participants want more than "lip service" to increasing diversity and promoting inclusivity-they want action. This desire for action rather than rhetoric or superficial reactions is similar to much of the interview data [2].

In 40 years, hasn't changed. (INT:P102:F-42:58)

Dealing with these issues is exhausting. (S3Q12:1644:F)

The interview and open-ended data generally highlight a sense of resignation on the part of participants who have not seen any real change and who are "exhausted" at having to deal with these issues. 
While many open-ended responses question NIST's commitment to increasing diversity and promoting inclusivity, a number of survey participants referred to the ways in which gender bias and discrimination are a thing of the past or related to the age of men working at NIST, and that it is improving as older men retire.

NIST is getting better about gender issues one retirement at a time. (S3Q12:P1220:M)

The bias and misogyny I have heard about are in the past and involve a few older men in NIST management who grew up in a different time. I have not personally experienced or witnessed such things at NIST although I did at other places I worked in the past. (S3Q12:P1782:F)

The gender-dependent behaviors I've seen appear to be strongly correlated with staff age. At the time that I witnessed these behaviors (early 2000s), the female victims were typically in their 30 s or even $20 \mathrm{~s}$, while the male instigators were in their $50 \mathrm{~s}$ or $60 \mathrm{~s}$. (S3Q12:P1768:M)

In the past, it appeared that there were disparities regarding equal pay and promotions for woman in favor of male counterparts. Currently, the opposite it appears to be true. There is greater push promote and advance females over equally or more qualified males. We should be evaluating the quality of work not the gender, race, etc of individual completing the work. (S7Q14:P1982:F)

Most of my experiences with being treated differently were associated with a previous supervisor. The work environment has definitely improved in recent years and as some staff with outdated views have retired or left. (S3Q12:P1587:F)

These participants see gender discrimination as the result of one person's actions, usually older white men, and not as a systemic problem built into the culture. This is one mechanism by which systemic discrimination is allowed to continue-by seeing it as related to one man, or one generation, rather than as built into a system that privileges some more than (or at the expense of) others. While it may be true that much of the specific acts of discrimination are committed by "older men," it does not mean that the culture itself is not imbued with oppressive policies, practices, and beliefs that also work to perpetuate inequities.

The majority of responses identified the systemic nature of the problems and the need for systemic change that addresses the organization as a whole and its culture. This was seen in the interview data as well as in the open-ended survey data.

But for some reason, we still-- again, that's that meritocratic problem where we still are thinking things in a build-the-numbers-per-person case instead of build the impact for the organization. As long as we still have that part, we're going to have a lot of these other systematic problems. (INT:P103:M-1:07:43)

A recognition that I think there is a cultural-- I'm going to use the word systemic, and that's too big of a word. But a systemic issue on the treatment of women at NIST. Maybe systemic isn't too big of a word. And that we as an organization have to do something to 
address it. And I don't-- there is no easy answer. And I completely recognize that. (INT:P104:F-57:54)

I would like to see the diversity and inclusivity move from a checkbox exercise to a true inclusive culture. (S1Q5:P2223:F)

The efforts seem largely superficial and designed to make NIST look good rather than solve the cultural issues that lead to a lack of inclusivity and diversity. (S1Q5:P2308:F)

Interview and survey participants both identified the need for broad cultural change at NIST in order to address the systemic nature of gender discrimination. One participant wrote eloquently, at length about how the very terminology of diversity and inclusivity make it difficult for an organization, and its leadership, to understand and address the systemic nature of racism and sexism.

"Inclusivity" and "diversity" are politically correct code words that make it difficult for anyone, including our leadership, to understand the systemic and institutional racism and sexism that pervade our culture at NIST. There is no discussion about implicit bias. There is no discussion of White as the assumed "norm" for race that does not require naming, and anything else as requiring designation as Black, Latinx, Asian, or any other racial characterization used to designate people of color as different from White. It is particularly discouraging that you are using a staff survey to query a predominantly White male staff in order to determine if NIST can give itself a pass with regard to sexism and racism. Of course, the typical White male will not consider race and gender to be a problem at NIST, (outside of hiring statistics, perhaps,) precisely because White males are so advantaged due to their race and gender that they consider their experience to be the norm. The very nature of this survey assumes that we can use a color- and gender-blind approach to build a statistical portfolio of NIST's success in addressing sexism and racism. However, Color-blind and gender-blind approaches to ending racism and sexism only play into the adaptability of the system of oppression that is white supremacy. To end such a system of oppression, we must name the key ideologies that support white supremacy in our country: Individualism and Meritocracy. Unfortunately, those ideologies are so deeply imbedded into the culture at NIST that I despair that we will ever achieve the level of self-awareness required to actually undo the insidious workings of institutional racism and implicit bias in the sciences, in general, and within NIST, in particular. Indeed, all I need to do is look at the race and gender mix of NIST higher management (Lab Directors and Division Chiefs, for example) and it is all too apparent that our institution still buys into the many norms that are the foundational support of racism and sexism. I know that we have some women and POC in upper level management positions, but these are still so strongly skewed as minorities that they continue to have the appearance of "token" hires intended to take the pressure off of management to increase "diversity" and "inclusivity", but don't force us as an institution 
to take the difficult steps of humble education and honest introspection that are required to make really progress on this problem. (S1Q5:P2182:M)

His words speak volumes about the cultural foundations of systemic oppression as well as its effects.

\subsubsection{Potential Leadership Actions}

Interview participants from Phase 2 identified targeted actions they would like to see as outcomes from this study [2]. Likewise, some survey participants highlighted specific things NIST leadership could do to advance efforts to increase diversity and promote inclusivity in their open-ended responses.

There has been a small amount of positive messaging from leadership, but I have not seen any concrete actions taken to improve the situation. If leadership wants to encourage work in this direction, wording needs to be included in performance plans which rewards efforts related to inclusivity. Scientists should be encouraged to actively recruit a broad spectrum of talent and more direct outreach should occur to HBCUs and other minority institutions. Leadership should seek outside expertise for guidance. (S1Q5:P2193:M)

There needs to be a much greater emphasis on attracting, retaining, and promoting women and minorities. This can include aggressive outreach to HBCUs and traditional women's colleges, particularly with the SURF and postdoc programs which are an important way in which young scientists become aware of NIST. (S1Q5:P2128:F)

Not only does NIST need to take concrete action, it needs to commit resources to efforts aimed at increasing diversity and promoting inclusivity.

We got to put our money where our mouth is. (INT:P102:F-1:11:00)

Simply put, there are minimal to no efforts by the senior leadership and/or the Associate Directors. It comes off as nothing but lip service. There has yet to be a clearly articulated goal by management for what diversity and inclusion changes need to be made. There are zero resources truly dedicated to diversity and inclusion. The senior management does not hold Lab Directors, Div Chiefs, and Group Leaders accountable for being transparent with processes. Senior leadership and the AD's often appear to only go after the "low hanging fruit" when it comes to diversity and inclusion efforts. As a result nothing has changed in my $10+$ years here. My direct supervisor does however promote an inclusive environment and their team is extremely diverse. The senior leadership team needs to do a better job at fixing the current issues (salary disparities , promotion potential etc) and set the stage for future employees to come into an inclusive work environment. Further, senior management only appears to want to address gender inequities yet constantly sweeps the inequities tied to race under the rug. (S1Q5:P1647:F) 
Without allocating resources to address diversity and inclusivity, real change remains elusive. There is also an understanding that this type of cultural change takes time, because it is in fact systemic, and not solely based on individual actions.

Meaningful and lasting change won't be quick or easy. I'm glad to see that NIST is starting this process. It will require a sustained effort from all areas of NIST. Top down and bottom up. (S1Q5:P2092:F)

And so, yes, to me an outcome of a recognition that we actually have a problem, an acceptance of the fact that there's a problem. I don't expect anyone to come forward with an easy solution. It doesn't have one. But I do think, yeah, a recognition, an acceptance, and a willingness to say, "We'll make changes. We don't know what they are yet. And we might make some changes that don't help it. We might try things." But I think a willingness to say, "We're actually going to address it and make changes as a result." So that'd be \{mine?\}. (INT:P104: F - 59:04)

In order to address systemic forms of oppression, the organization must admit to often difficult truths about itself. It must also recognize that there are no easy answers, no fast solutions.

Overarchingly, the qualitative data from both interviews and open-ended responses indicate that participants do not believe NIST leadership is doing, or has done, enough to increase diversity and promote inclusivity. Recognizing the systemic nature of the problem is just the first step.

\section{Discussion}

Across the datasets there is an overwhelming theme and feeling of frustration. The word frustration appears in the responses, as do the feelings that accompany it. Women are frustrated by the Chilly Climate they continue to experience - the lack of acceptance, the lack of opportunity, the assumptions about their work and their ability, the microaggressions and the list goes on. Men are frustrated by these as well. Many participants are frustrated by the sole focus on gender to the perceived exclusion of race, sexuality, or other issues related to diversity and inclusion. They are also frustrated by the slow pace of change, many believing that there really has been very little change over the years, despite the rhetoric. Some men are frustrated that this study is even being done at all, and believe that it is not needed, sometimes describing their perceptions about reverse discrimination.

Women are tired, tired of the continuous struggle, tired of defending their "right to be here," to be heard, and participate.

I have not spent my whole career at NIST and have seen first hand that other organizations are more diverse and inclusive. While I love my work at NIST, there are times when the lack of understanding of issues around diversity and inclusivity is tiring. (S6Q3:1592:F) 
Physics is deemed the most valuable research endeavor at NIST. Doing bio-related projects in PML is not valued and I'm really tired. And, the lack of commitment by OU leadership to actually learn about Bio in PML is tiring. I'm also tired of all the big egos and infighting for funding. Everyone has to be the smartest person in the room, which is tiring too. And, the emphasis on "only bring me solutions" is ridiculous. First, you have to identify and bring a problem to leadership's attention. Putting the burden of finding a solution on the people who experience the less-than-ideal interactions is not acceptable. (S6Q3:1820F)

So this is the tilting at windmills thing again. I get frustrated and I sometimes have a short wick. Because it's like, "I've had this conversation. I've had this conversation. I've had this conversation. I'm having this conversation again. Oh, my gosh." And a lot of it is pushing against inertia. Like, "Why can't we fix these problems that we have?" "Oh, because we don't have money. We don't have enough time." These are excuses. If this is a priority, we shift other things and we do this. (INT:P103:F-19:32)

\section{Women at NIST are suffering from "gender fatigue" Kelan [23]. The toll of energy expended to acknowledge and oppose gender discrimination has become too much. Many women are frustrated by the apparent futility of it all.}

NIST is mostly talk, as far as I have seen in my decade here. Leadership SAYS all the right things, and does support the grassroots groups in a nominal or very individual way (Pride, ANAPA, WISTEM). However, actual, actionable changes have not come forth. NIST is obsessed with studies, metrics, and data, rather than obsessing with PEOPLE. The studies have all been done before; the metrics have all been taken before; the data will tell you what any minority at NIST already knows: being "different" than white/male/STEM/academic gets you punished here. Not overtly of course. But implicitly. Difference is not understood, celebrated, or desired. The myth of the meritocracy is so entrenched here that even well-meaning individuals cannot change the general attitude of leadership - "There's nothing we can do if there's no x minorities in STEM" or "It's not MY responsibility or obligation to intentionally make this a safe space for women/BIPOC." Until the mid-level management (lab directors, div chiefs) get onboard and set the example through ACTION (not just words), NIST is not going to change. I would never recommend any of my young female/BIPOC friends come and work at NIST. It's not a friendly environment to them and they'll have to spend all their time defending their right to be here. (S1Q5:1454:F)

I mean, my goodness, who would want to be the one to rock the boat, especially if you are are junior staff, especially if you do not have tenure, and ESPECIALLY if the people who are doing this have absolute power to make your life miserable? At the end of the day, this is our livelihood, we have family members who depend on our income. We endure because we have to. I would like to change that within the system as I age into it. Until you change that power dynamics, nothing will change. (S3Q12:1702:F) 
The "gender fatigue" and the necessary time and energy required to make change results in a sense of resignation - that nothing will happen, so why bother.

Other disenfranchised groups are frustrated that the focus on diversity and inclusion at NIST appears to be primarily on women and not race or on a broader view of gender-transgender or other LGBTQ+ individuals. Several participants expressed disappointment that the focus of this research study on gender was specifically on women and was too narrow.

I am extremely disappointed by the narrow description of inclusivity in this survey. I am aware that there is a much higher number of men than women at NIST Boulder, and we definitely need to do something about it, so I commend NIST for working on that issue. However, diversity is not only defined by gender, there is almost no race diversity at NIST Boulder and I haven't seen much effort from leadership to fix this huge issue. The black and latino communities are almost non existent in the Boulder campus and of the few scientists of black/latino descent, none are in leadership positions. With the exception of some outreach from the now director of CTL, I haven't seen any efforts to attract under-represented communities. Even when people do some outreach, it is seen as a waste of time rather than a part of our job to make NIST a more inclusive institution. I believe that our job as NIST scientists is not only to focus on science, but to improve society overall, and that includes outreach activities. When this study was publicized, I attended the meeting in Boulder and was very disappointed to hear that inclusivity was only defined by gender. As a member of these underrepresented communities, I hope NIST expands the study to also include race, not just for the overall health of NIST, but also for the scientific community. (S1Q5:1678:M)

NIST is too focused on "gender and inclusivity" to mean "female empowerment." Where is the representation and focus on transgender or other LGBTQ+ individuals' needs? It's pretty much entirely absent. I would like women to be empowered here too, being one and all, but I am afraid my transgender or otherwise gender non-conforming coworkers aren't even being asked to come to the table. (S6Q4:1454:F)

Many responses identified other characteristics including race and sexuality, and other dimensions of identity such as ethnicity, religion, language, and culture. Intersectional theory recognizes that these multiple characteristics do not exist independently each informing the other and creating a complex convergence of bias [28]. One participant was frustrated that the intersectionality of race and gender is not a focus.

NIST always seems to focus on gender but never looks at the intersectionality that comes into play due to my race. I would've selected more "way more than once" if my race/gender was the topic of the question. (S3Q12:1647:F)

While most participants recognized the importance and need for diversity and inclusivity, others expressed frustration with the emphasis on diversity and inclusivity at all.

Again, God and science make the concept of gender clear; there are two: male and female. Anything else is a mutation of one or the other. I tire of getting emails about "first 
woman to..."; so what. I tire of invites to events exclusive to females. (They are oxymoronic for those who embrace gender fluidity, diversity, and inclusiveness.) I tire of LGBTQIA++++++ celebratory emails. I tire of the federal government providing special protections not available to me. I tire of the workplace surveys that even acknowledge the idiocy that is anything other than male and female. (S3Q12:1931:M)

As MLK espoused: people should "not be judged by the color of their skin but by the content of their character." Hiring decisions should be NOT be made based on someones skin color, religious affiliation, or ethnicity, but rather on their qualifications and their character. Anything other then this is discriminatory and self-defeating, whether to the minority or non-minority. (S1Q5:1245:M)

Maybe I don't understand the method and/or end goal well enough. But I don't see how focusing on innate characteristics like skin color and gender over knowledge, skills, and abilities is fair and will help us meet our organizational goals? I have heard this is only used as a small piece of the puzzle, but have seen in practice innate characteristics being the main factor once minimum qualifications are met, regardless of any other metric. (S1Q5:1526:M)

It's probably no worse than anywhere else. (S6Q4:1674:M)

While these participants questioned the need and emphasis on diversity and inclusivity, some men expressed their frustration that there is even a focus on diversity and inclusivity. Terms like threatening, offensive, and reverse discrimination were used to describe their frustration.

I am deeply offended by the very first question in this survey, "I identify as a man, woman, other" THERE IS NO OTHER!!! You are either a man or a woman, God did not make more than male and female. I think this whole thing of inclusivity and diversity is a bunch of bunk and a total waste of time, effort, and money. I person should be included because they are a human being and the only diversity we need is as simple as men and women. Nothing else. (S1Q5:1552:M)

If you mean by including different perspectives; no. If you mean rewarding people based upon their skin color, so-called gender fluidity, or other politically correct characteristics; yes. Actually, for a scientific group of people they follow the social tyrants instead of the science. NIST needs to stop all this divisive, wasteful foolishness. Diversity by definition is divisive. Wonder why we call Universities, Universities...not Diversities. Because diversity is not a virtue in the U.S. Look up E Pluribus Unum; the original meaning not the politically correct bastardized version. Diversity and inclusion are the modern terms for affirmative action/quotas. In essence, reverse discrimination against people who have no control over the color of their skin being white. If NIST were interested in true diversity and inclusivity it would have the staffing mirror the population with $75 \%$ white, $18 \%$ Hispanic, $13 \%$ Black-skinned, Asian 5\%, and the other minorities per their 
percentage. Per God (and affirmed by science), there is only one Race...the human race. Hiring ought to be based upon merit, not ethnic, gender, geography, etc. (S1Q5:1931:M)

While some men are frustrated by what they perceive as reverse discrimination other participants expressed frustration with the actual research effort itself.

Most attempts at 'inclusion' at NIST are focused on *signaling* inclusivity, rather than actually *being* inclusive. For example, I think that the detail-appointment-based approach to improving gender equity at NIST is a disappointing gesture, because the participants have not been given enough time or power to make change on the scale that is needed. (I believe that the participants genuinely want to improve NIST - this is an organization/institution-level failure.) One example of how this manifests is in this survey: the first question asks about gender identity in an outdated way that fails to capture a nuanced range of possible responses, and requires anyone whose identity is not easily categorized as a man or a woman to quite literally other themselves. Also, I am a nonbinary transgender person and at no point during my two year NRC fellowship did I feel safe coming out to anyone on my management chain or in leadership.

(S1Q5:1892:O)

"doing enough" is leading language. There is no room in this survey to question whether the merit of "increasing diversity" is properly balanced against other concerns. There is no answer offered where I can record an opinion that too much attention is being paid to these matters, and that other important matters, even legal obligations, are being shortchanged as a consequence. The biased frame of the questions suggest to me an element of hostility to certain perspectives. Not what you want in an honest survey. (S1Q5:2477:M)

Who wrote this question? Stop looking at inclusivity and diversity through the lens of gender and color. It is a failed and tired trope. (S1Q5:1663:M)

But, the most frustrating aspect of diversity and inclusion efforts at NIST appears to be the slow pace of change. Participants, both men and women, used terms like lip-service, a lot of talk and no action, smoke and mirrors, talk the talk but don't walk the walk, and "the subject is being NISTed to death, i.e. stuck in some paralyzing, perfectionist cycle" (S1Q5:2516:F) to refer to the lack of progress and actions.

When it comes to diversity and inclusivity, there always has to be a study to conclude what we already know is happening. These studies seem to be stall tactics to postpone actually taking real action. This is the reason for my answers above. It's like the house voting on whether or not African Americans should be granted reparations for systemic racism. Of course there should be reparations! What is there to study! I feel the same way about what NIST is doing. (S1Q5:2148:F)

We've heard a lot of talking but I cannot name real, meaningful actions being taken by the leadership. I understand a culture that is deeply rooted takes time to change. But 
without actions, more conversations will simply reinforce the perceptions that we are just checking boxes here. (S1Q5:1311:M)

Simply put, there are minimal to no efforts by the senior leadership and/or the Associate Directors. It comes off as nothing but lip service. There has yet to be a clearly articulated goal by management for what diversity and inclusion changes need to be made. There are zero resources truly dedicated to diversity and inclusion. The senior management does not hold Lab Directors, Div Chiefs, and Group Leaders accountable for being transparent with processes. Senior leadership and the AD's often appear to only go after the "low hanging fruit" when it comes to diversity and inclusion efforts. As a result nothing has changed in my $10+$ years here. My direct supervisor does however promote an inclusive environment and their team is extremely diverse. The senior leadership team needs to do a better job at fixing the current issues (salary disparities, promotion potential etc) and set the stage for future employees to come into an inclusive work environment. Further, senior management only appears to want to address gender inequities yet constantly sweeps the inequities tied to race under the rug. (S1Q5:1647:F)

The feelings of frustration permeate the data - from women who are frustrated by a culture where microaggressions, unwanted physical attention and gendered harassment still exist to women and men who are frustrated with the slow pace of change. From others who are frustrated by the focus on gender and not race or sexual identity to some that are frustrated by the focus on diversity and inclusivity at all. How can employees be productive and focused on NIST's mission if all this time and energy is spent being frustrated?

\section{Conclusions}

What does diversity mean in the context of this report? We have collected a great deal of data across the phases of this research project. We have numbers from the HR data analysis that demonstrate we are not diverse. We have qualitative data from in-depth interviews and quantitative and qualitative data from the surveys, again demonstrating that the organization is not diverse and inclusive. Numbers are important, a first step against which to measure progress. Yes, we need to hire and promote more women. But the numbers are not the only metric.

The qualitative data point to the culture as the root of the problem. The lack of diversity is a reflection of the overall culture of NIST. Increasing the numbers of women and minorities may help us look more representative of the broader community but we need to address the culture and establish a culture that celebrates diversity and inclusivity - a culture where everyone is respected, valued, accepted, and treated equally. Practices, processes and procedures for increasing diversity can be in place, but if the underlying culture is not in place to support inclusivity, it's irrelevant.

Throughout this report, discrimination and gender oppression are discussed as systemic and embedded in the culture and not solely the acts of individuals. The discussion does not imply 
that all women and men fall into these characterizations or categories and believe these tropes. However, we must collectively acknowledge the ways in which sexism does operate on a systemic level. Recognizing the systemic nature of the problem is the first step.

Everyone at NIST must contribute to and participate in the cultural changes required to underpin a diverse and inclusive environment. Men and women need to be part of the solution; leadership and staff need to be part of the solution - the changes need to be farreaching and organization-wide. As P2182 notes in a preceding section, what is really needed is "to take the difficult steps of humble education and honest introspection that are required to really make progress on this problem." This work is difficult, takes time, and does not rely on easy solutions, but if we at NIST are willing to do the hard work, we have an opportunity to make real progress. In spite of overwhelming frustration we are moving toward a place of hope - this can be the beginning of the hard work of "honest introspection." 


\section{Acknowledgments}

We would like to acknowledge and thank those who have made this work possible. First, we would like to acknowledge the Associate Director of Laboratory Programs, Dr. James Olthoff, for the funding support and opportunity to perform this research. Additionally, we would like to thank the 40 employees who took time out of their busy schedules to participate in the in-depth interviews and share their experiences of working at NIST and all the federal employees who graciously gave their time and input participating in the survey. Finally, thanks to the collaborators of the first three documents Jasmine Evans, Susanne Furman, Amanda Koepke, Steve Lund, and Justyna Zwolak and a special thanks to Susanne Furman in helping out at multiple junctures with this document. 


\section{References}

1. $\quad$ Evans, J., Koepke, A., Lund, S.P., Theofanos, M.F. , Examining Recent HR Data for Gender Bias Among Federal Employees at NIST. NIST IR 8363, March 2021, National Institute of Standards and Technology.

2. Theofanos, M., Prettyman, S., Evans,J., Furman, S., Voices of NIST: A Study of Gender and Inclusivity, Findings from In-depth Interviews. NIST TN 21143, March 2021, National Institute of Standards and Technology.

3. Theofanos, M.F., Evans,J., Zwolak, J.P., Prettyman, S., Survey on Gender, Equity and Inclusion. NIST IR 8362, March 2021,

4. $\quad$ Fetters, M.D., L.A. Curry, and J.W. Creswell, Achieving integration in mixed methods designs - principles and practices. Health services research, 2013. 48(6pt2): p. 2134-2156.

5. Creswell, J.W. and V.L.P. Clark, Designing and conducting mixed methods research. 2017: Sage publications.

6. Britton, D.M., Beyond the chilly climate: The salience of gender in women's academic careers. Gender \& society, 2017. 31(1): p. 5-27.

7. Settles, I. Women in STEM:Challenges and determinants of success and well-being. 2014; Available from: https://www.apa.org/science/about/psa/2014/10/women-stem.

8. Baer, M., et al., Intergroup Competition as a Double-Edged Sword: How Sex Composition Regulates the Effects of Competition on Group Creativity. Organization Science, 2014. 25(3): p. 892-908.

9. $\quad$ Fang, F.C., A. Casadevall, and A.J. Bäumler, Competitive Science: Is Competition Ruining Science? Infection and Immunity, 2015. 83(4): p. 1229-1233.

10. Farenga, S.J. and B.A. Joyce, Intentions of young students to enroll in science courses in the future: An examination of gender differences. Science Education, 1999. 83(1): p. 55-75.

11. Hill, C., C. Corbett, and A. St Rose, Why so few? Women in science, technology, engineering, and mathematics. 2010: ERIC.

12. Daniels, N., Merit and meritocracy. Philosophy \& Public Affairs, 1978: p. 206-223.

13. Perez, C.C., Invisible women: Exposing data bias in a world designed for men. 2019: Random House.

14. Johnson, S., Inclusify: The Power of Uniqueness and Belonging to Build innovative Teams. 2020, New York: Harper Collins.

15. Kahneman, D., Thinking, fast and slow. 2011: Macmillan.

16. Exley, C. and J. Kessler, Why Don't Women Self-Promote As Much As Men? Harvard Business Review, 2019.

17. Cahn, N. Women Don't Sefl-Promote-And Why It Matters So Much. Forbes 2019 [cited 2021 January 15, 2021]; Available from: https://www.forbes.com/sites/naomicahn/2019/12/18/lessons-in-selfpromotion/?sh=59f6fe37630c.

18. National Academies of Sciences, E. and Medicine, Promising Practices for Addressing the Underrepresentation of Women in Science, Engineering, and Medicine: Opening Doors. 2020: National Academies Press. 
19. National Academies of Sciences, E. and Medicine, Sexual harassment of women: climate, culture, and consequences in academic sciences, engineering, and medicine. 2018: National Academies Press.

20. Cheny, I.a.S.S., Picture a Scientist. 2020.

21. Cech, E.A. and M. Blair-Loy, The changing career trajectories of new parents in STEM. Proceedings of the National Academy of Sciences, 2019. 116(10): p. 41824187.

22. Grissinger, M., Disrespectful behavior in health care: its impact, why it arises and persists, and how to address it—part 2. Pharmacy and Therapeutics, 2017. 42(2): p. 74.

23. Kelan, E.K., Gender fatigue: The ideological dilemma of gender neutrality and discrimination in organizations. Canadian Journal of Administrative Sciences/Revue Canadienne des Sciences de l'Administration, 2009. 26(3): p. 197-210.

24. Thomas, D.A. and R.J. Ely, Making differences matter: A new paradigm for managing diversity. Harvard business review, 1996. 74(5): p. 79-\&.

25. Center, A.C.L.R. The Myth of Reverse Racism 2021; Available from: http://www.aclrc.com/myth-of-reverse-racism.

26. Brod, H. and M. Kaufman, Theorizing masculinities. Vol. 5. 1994: Sage Publications.

27. Jorgenson, J., Engineering selves: Negotiating gender and identity in technical work. Management Communication Quarterly, 2002. 15(3): p. 350-380.

28. Cho, S., K.W. Crenshaw, and L. McCall, Toward a field of intersectionality studies: Theory, applications, and praxis. Signs: Journal of women in culture and society, 2013. 38(4): p. 785-810. 


\section{Appendix A: Survey Notation and Numbering}

\begin{tabular}{|c|c|c|c|c|c|}
\hline \multicolumn{6}{|c|}{ Section One: Commitment to Inclusivity \& Diversity } \\
\hline \multicolumn{6}{|c|}{ How much do you agree or disagree with the following statements? } \\
\hline & Reference & $\begin{array}{l}\text { Strongly } \\
\text { Disagree }\end{array}$ & Disagree & Agree & $\begin{array}{l}\text { Strongly } \\
\text { Agree }\end{array}$ \\
\hline $\begin{array}{l}\text { Q1: Senior Leadership is } \\
\text { committed to inclusivity. }\end{array}$ & S1Q1 & & & & \\
\hline $\begin{array}{l}\text { Q2: Senior Leadership is } \\
\text { committed to increasing diversity in } \\
\text { the workplace. }\end{array}$ & $\mathrm{S} 1 \mathrm{Q} 2$ & & & & \\
\hline $\begin{array}{l}\text { Q3: My OU is doing enough to } \\
\text { increase diversity in the workplace. }\end{array}$ & S1Q3 & & & & \\
\hline $\begin{array}{l}\text { Q4: My OU is doing enough to } \\
\text { promote inclusivity. }\end{array}$ & S1Q4 & & & & \\
\hline
\end{tabular}

Section Two: Perceptions of NIST Culture

How much do you agree or disagree with the following statement?

\begin{tabular}{|c|c|c|c|c|c|}
\hline & Reference & $\begin{array}{l}\text { Strongly } \\
\text { Disagree }\end{array}$ & Disagree & Agree & $\begin{array}{c}\text { Strongly } \\
\text { Agree }\end{array}$ \\
\hline $\begin{array}{l}\text { Q1: ....the best opportunities go to } \\
\text { the most deserving employees. }\end{array}$ & S2Q1 & & & & \\
\hline $\begin{array}{l}\text { Q2: ...opportunities are given } \\
\text { based on who you know. }\end{array}$ & S2Q2 & & & & \\
\hline $\begin{array}{l}\text { Q3: ....staff who have been at } \\
\text { NIST as long time listened to more } \\
\text { than staff } \\
\quad . . \text { for shorter time. }\end{array}$ & S2Q3 & & & & \\
\hline $\begin{array}{l}\text { Q4: ....teamwork is valued just as } \\
\text { much as individual performance. }\end{array}$ & S2Q4 & & & & \\
\hline
\end{tabular}




\begin{tabular}{||l|c|l||}
\hline $\begin{array}{l}\text { Q5: ....teamwork is rewarded just } \\
\text { as much as individual } \\
\text { performance. }\end{array}$ & S2Q5 & \\
$\begin{array}{l}\text { Q6: ....that projects are seen as } \\
\text { more important than people. }\end{array}$ & S2Q6 & \\
\hline $\begin{array}{l}\text { Q7: ...that NIST values scientists } \\
\text { over staff. }\end{array}$ & S2Q7 & \\
$\begin{array}{l}\text { Q8: ....that NIST believes itself to } \\
\text { be more objective than subjective }\end{array}$ & S2Q8 & \\
\hline $\begin{array}{l}\text { Q9: Do you have any additional comments about NIST Culture that you would like to } \\
\text { include? (S2Q9) }\end{array}$ \\
\hline
\end{tabular}

\begin{tabular}{|c|c|c|c|c|}
\hline \multicolumn{5}{|c|}{ Section Three: Gendered Experiences } \\
\hline \multicolumn{5}{|c|}{$\begin{array}{l}\text { Consider the following statements specifically in relationship to gender. Do you believe } \\
\text { you have experienced any of the following while working at NIST? }\end{array}$} \\
\hline & Reference & Yes & No & I don't know \\
\hline $\begin{array}{l}\text { Q1:...I have been interrupted in a meeting } \\
\text { because of my gender. }\end{array}$ & S3Q1 & & & \\
\hline $\begin{array}{l}\text { Q2: .... have been excluded from } \\
\text { meetings/email or other work-related } \\
\text { activities. }\end{array}$ & S3Q2 & & & \\
\hline $\begin{array}{l}\text { Q3: ....others have taken credit for my } \\
\text { ideas/work. }\end{array}$ & S3Q3 & & & \\
\hline $\begin{array}{l}\text { Q4: ....my judgement questioned in my area } \\
\text { of expertise. }\end{array}$ & S3Q4 & & & \\
\hline $\begin{array}{l}\text { Q5:...I have missed out on work } \\
\text { opportunities. }\end{array}$ & S3Q5 & & & \\
\hline Q6: ....I have been treated differently. & S3Q6 & & & \\
\hline $\begin{array}{l}\text { Q7: .... have witnessed women and men } \\
\text { being treated differently. }\end{array}$ & S3Q7 & & & \\
\hline $\begin{array}{l}\text { Q8: ...I have read, heard, and/or seen } \\
\text { insensitive comments about gender in my } \\
\text { work unit that I found offensive. }\end{array}$ & S3Q8 & & & \\
\hline Q9: ....I have felt isolated in my work. & S3Q9 & & & \\
\hline $\begin{array}{l}\text { Q10a: ...I have experienced harassment } \\
\text { because of my gender. }\end{array}$ & S3Q10 & & & \\
\hline
\end{tabular}


Q10b: ...I have experiences harassment because of my gender.

Q11: ...I am aware of others who have experiences harassment.

Q12: Do you have any additional comments about your experience at NIST based on gender that you would like to include? (S3Q12)

\begin{tabular}{|c|c|c|c|c|c|}
\hline \multicolumn{6}{|c|}{ Section Four: Gendered Outcomes: Interactions 1} \\
\hline \multicolumn{6}{|c|}{ To what extent do you agree or disagree with the following statements I believe... } \\
\hline & Reference & $\begin{array}{l}\text { Strongly } \\
\text { Disagree }\end{array}$ & Disagree & Agree & $\begin{array}{l}\text { Strongly } \\
\text { Agree }\end{array}$ \\
\hline $\begin{array}{l}\text { Q1: ....I have to work harder than my } \\
\text { colleagues for my work to be } \\
\text { recognized/accepted... }\end{array}$ & S4Q1 & & & & \\
\hline $\begin{array}{l}\text { Q2: .... was not considered ready for } \\
\text { promotion due to my gender } \\
\text { rather than the quality of my } \\
\text { work. }\end{array}$ & S4Q2 & & & & \\
\hline $\begin{array}{l}\text { Q3: ....the way I dress influences } \\
\text { others' view of my competence. }\end{array}$ & S4Q3 & & & & \\
\hline $\begin{array}{l}\text { Q4: People I work with listen to my } \\
\text { ideas when making decisions. }\end{array}$ & S4Q4 & & & & \\
\hline $\begin{array}{l}\text { Q5: My work at NIST is done alone } \\
\text { rather than working with others. }\end{array}$ & S4Q5 & & & & \\
\hline $\begin{array}{l}\text { Q6: Overall, I feel I "fit" in with } \\
\text { colleagues I work with. }\end{array}$ & S4Q6 & & & & \\
\hline $\begin{array}{l}\text { Q7: I would like to be in leadership at } \\
\text { NIST. }\end{array}$ & S4Q7 & & & & \\
\hline $\begin{array}{l}\text { Q8: Women and men have equal } \\
\text { opportunity to move into leadership }\end{array}$ & S4Q8 & & & & \\
\hline $\begin{array}{l}\text { Q9: I believe I would be accepted and } \\
\text { respected as a leader at NIST... }\end{array}$ & S4Q9 & & & & \\
\hline
\end{tabular}




\begin{tabular}{|l|c|c|c|c|}
\hline \multicolumn{4}{|c|}{ Section Five: Gendered Outcomes: Interactions 2} \\
\hline Think about how often you have the following interactions. \\
\hline $\begin{array}{l}\text { Q1: I have substantive conversations } \\
\text { with colleagues. }\end{array}$ & S5Q1 & & \\
\hline $\begin{array}{l}\text { Q2: I have conversations with my } \\
\text { First Line Supervisor. }\end{array}$ & S5Q2 & & \\
\hline $\begin{array}{c}\text { Q3: I have conversations with my } 2^{\text {nd }} \\
\text { year }\end{array}$ & S5Q3 & $\begin{array}{c}\text { Sometimes } \\
\text { (at least } \\
\text { once/month) }\end{array}$ & $\begin{array}{c}\text { Frequently } \\
\text { (at least once } \\
\text { week) }\end{array}$ \\
\hline $\begin{array}{l}\text { Line Supervisor (like Div Ch or OU Dir) } \\
\text { Q4: I have conversations with my } \\
\text { OU Director }\end{array}$ & S5Q4 & \\
\hline
\end{tabular}

\begin{tabular}{|c|c|c|c|}
\hline \multicolumn{4}{|c|}{ Section Six: Gendered Outcomes: Interactions 3} \\
\hline \multicolumn{4}{|l|}{ Consider the following statements. } \\
\hline & Reference & Yes & No \\
\hline $\begin{array}{l}\text { Q1: I have had formal/informal mentors who have helped } \\
\text { me be successful at NIST. }\end{array}$ & S6Q1 & & \\
\hline$+1 \mathrm{a}$ (through a formal program) & S6Q1a & & \\
\hline $\begin{array}{l}\text { Q2: I have been a formal/informal mentor to others at } \\
\text { NIST. }\end{array}$ & S6Q2 & & \\
\hline$+2 \mathrm{a}$ (through a formal program) & S6Q2a & & \\
\hline $\begin{array}{l}\text { Q3: ...considered leaving NIST for reasons related to } \\
\text { diversity? }\end{array}$ & & & \\
\hline $\begin{array}{l}\text { Q4: Do you have any additional comments about the Wor } \\
\text { would like to include? (S6Q4) }\end{array}$ & nvironme & it N] & \\
\hline
\end{tabular}




\begin{tabular}{|c|c|c|c|c|c|}
\hline Section Seven: Gena & ered Outcom & s: Beliefs c & bout NIST & & \\
\hline To what extent do you agree or disagre & e with the fo & owing statc & ements. At & NIST: & \\
\hline & Reference & $\begin{array}{l}\text { Strongly } \\
\text { Disagree }\end{array}$ & Disagree & Agree & $\begin{array}{c}\text { Strongly } \\
\text { Agree }\end{array}$ \\
\hline $\begin{array}{l}\text { Q1: Women and men have equal } \\
\text { opportunity to be hired. }\end{array}$ & S7Q1 & & & & \\
\hline $\begin{array}{l}\text { Q2: Women and men have equal } \\
\text { opportunity for advancement. }\end{array}$ & S7Q2 & & & & \\
\hline $\begin{array}{l}\text { Q3: Women and men spend equal time } \\
\text { in a pay band before promotion. }\end{array}$ & S7Q3 & & & & \\
\hline $\begin{array}{l}\text { Q4: Compensation is fair for women } \\
\text { and men. }\end{array}$ & S7Q4 & & & & \\
\hline $\begin{array}{l}\text { Q5: The promotion process is applied } \\
\text { fairly and objectively. }\end{array}$ & S7Q5 & & & & \\
\hline $\begin{array}{l}\text { Q6: My need to balance work and } \\
\text { other life obligations is supported. }\end{array}$ & S7Q6 & & & & \\
\hline $\begin{array}{l}\text { Q7: Women...children are consider to } \\
\text { be }>\text { committed...than colleagues } \\
\text { who are not mothers. }\end{array}$ & S7Q7 & & & & \\
\hline $\begin{array}{l}\text { Q8: Men...children are considered to } \\
\text { be > committed...than colleagues } \\
\text { who are not fathers. }\end{array}$ & S7Q8 & & & & \\
\hline $\begin{array}{l}\text { Q9: Using available workplace } \\
\text { flexibilities...can result in } \\
\text { negative consequences for one's } \\
\text { career. }\end{array}$ & S7Q9 & & & & \\
\hline $\begin{array}{l}\text { Q10: Working long hours is an } \\
\text { important sign of commitment. }\end{array}$ & & & & & \\
\hline $\begin{array}{l}\text { Q11: Women and men are respected } \\
\text { equally. }\end{array}$ & & & & & \\
\hline $\begin{array}{l}\text { Q12: Women and men are valued } \\
\text { equally. }\end{array}$ & & & & & \\
\hline $\begin{array}{l}\text { Q13: Women and men are treated } \\
\text { equally. }\end{array}$ & & & & & \\
\hline $\begin{array}{l}\text { Q14: Do you have any additional comn } \\
\text { NIST, that you would like to include? }\end{array}$ & $\begin{array}{l}\text { ents about } \\
\text { (S7Q14) }\end{array}$ & way $\mathrm{m}$ & d won & re t & \\
\hline
\end{tabular}




\begin{tabular}{|l|c|c|c|c|}
\hline \multicolumn{3}{|c|}{ Section Eights: General Thoughts } \\
\hline Identify and Rank Your Top Three Choices \\
\hline $\begin{array}{l}\text { Q1: The greatest satisfaction I get from } \\
\text { working at NIST is: }\end{array}$ & Reference & Rank 1 & $\begin{array}{c}\text { Rank } \\
2\end{array}$ & Rank 3 \\
\hline $\begin{array}{l}\text { Q1a: do you have other things that give you } \\
\text { satisfaction from working at NIST? }\end{array}$ & S8Q1a & & \\
\hline $\begin{array}{l}\text { Q2: At NIST, people are respected more for } \\
\text { the following: }\end{array}$ & S8Q2 & & \\
\hline $\begin{array}{c}\text { Q2a: Are there other reasons people are } \\
\text { respected more at NIST? please list below: }\end{array}$ & S8Q2a & \\
\hline
\end{tabular}

